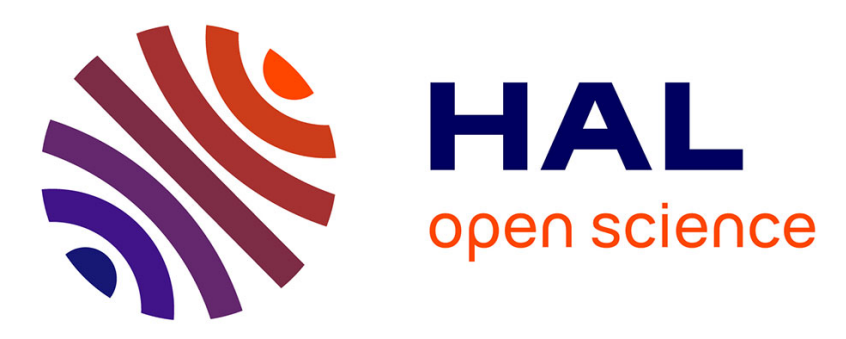

\title{
Au(I)-Catalyzed Pictet-Spengler Reactions all Around the Indole Ring
}

Pierre Milcendeau, Zhenhao Zhang, Nicolas Glinsky-Olivier, Elsa van

Elslande, Xavier Guinchard

\section{- To cite this version:}

Pierre Milcendeau, Zhenhao Zhang, Nicolas Glinsky-Olivier, Elsa van Elslande, Xavier Guinchard. $\mathrm{Au}(\mathrm{I})$-Catalyzed Pictet-Spengler Reactions all Around the Indole Ring. Journal of Organic Chemistry, 2021, 86 (9), pp.6406-6422. 10.1021/acs.joc.1c00270 . hal-03267801

\section{HAL Id: hal-03267801 \\ https://hal.science/hal-03267801}

Submitted on 22 Jun 2021

HAL is a multi-disciplinary open access archive for the deposit and dissemination of scientific research documents, whether they are published or not. The documents may come from teaching and research institutions in France or abroad, or from public or private research centers.
L'archive ouverte pluridisciplinaire HAL, est destinée au dépôt et à la diffusion de documents scientifiques de niveau recherche, publiés ou non, émanant des établissements d'enseignement et de recherche français ou étrangers, des laboratoires publics ou privés. 


\section{Au(I)-Catalyzed Pictet-Spengler Reactions all Around the Indole Ring}

Pierre Milcendeau, ${ }^{a}$ Zhenhao Zhang, ${ }^{a, b}$ Nicolas Glinsky-Olivier, ${ }^{a}$ Elsa van Elslande a and Xavier Guinchard*a

a Université Paris-Saclay, CNRS, Institut de Chimie des Substances Naturelles, UPR 2301, 91198, Gif-sur-Yvette, France.

${ }^{\mathrm{b}}$ LCM, CNRS, Ecole Polytechnique, Institut Polytechnique de Paris, 91128 Palaiseau, France.

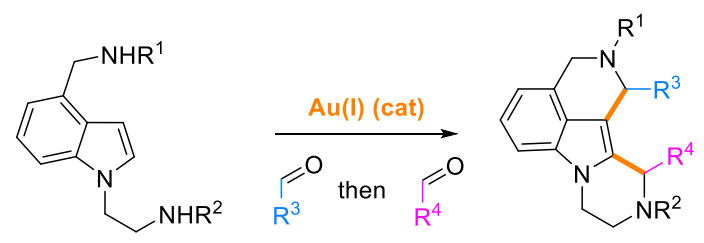

- $\mathrm{Au}(\mathrm{I})$ as the single catalyst • Good chemoselectivity

- Two consecutive iso-Pictet-Spengler reactions

ABSTRACT: $\mathrm{Au}(\mathrm{I})$ complexes catalyze iso-Pictet-Spengler reactions. Ethylamine or methylamine chains were introduced at $\mathrm{C2}, \mathrm{C} 4$ or at the nitrogen atom of the indole ring, and the corresponding substrates were reacted in the presence of aldehydes and catalytic amounts of $\mathrm{Au}(\mathrm{I})$ complexes, leading to a variety of polycyclic scaffolds. Selectivity could be achieved in the course of a double iso-Pictet-Spengler reaction involving two successive aldehydes, leading to highly complex molecules.

\section{INTRODUCTION}

Indole alkaloids are an important class of heterocycles because of their prevalence natural and bioactive compounds. ${ }^{1}$ Among these privileged scaffolds, tetrahydro- $\beta$-carbolines 1 are important molecules and their structural unit is embedded in numerous natural products, among which a huge number are bioactive. ${ }^{2}$ The Pictet-Spengler reaction ${ }^{3,4}$ combining tryptamines and aldehydes is unarguably the easiest and fastest way to prepare such scaffolds. ${ }^{2 b}$ Rich of more than a century of research, this reaction and its mechanism has been intensively studied ${ }^{5}$ and applied to numerous total syntheses. ${ }^{2 \mathrm{~b}}$ Interestingly, because of the nucleophilicity of indole at $\mathrm{C} 2$ and $\mathrm{C} 3,{ }^{6}$ numerous variants of the Pictet-Spengler reaction lead to structurally related compounds, such as 1,2,3,4-tetrahy-

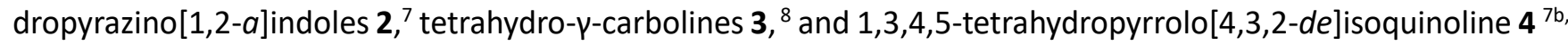
${ }^{9}$ (Figure 1). These so-called iso-Pictet-Spengler reactions are far less studied than the venerable historic reaction, despite allowing access to interesting heterocycles. ${ }^{10}$
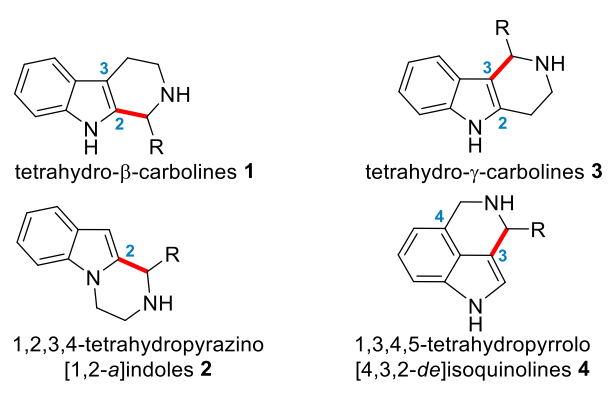

Figure 1. Tetrahydro- $\beta$-carbolines and congeners 
Our group has long been interested in the gold-catalyzed ${ }^{11}$ functionnalizations of indoles.$^{12,13}$ Recently, we discovered that Pictet-Spengler reactions could be catalyzed by $\mathrm{Au}(\mathrm{I})$ complexes $^{14,15}$ (Scheme 1, eq. 1). These reactions occur via a mechanism involving the auration of the indole ring that we established through both experimental and computational studies. We hypothesized that similar reactions catalyzed by $\mathrm{Au}(\mathrm{I})$ complexes should occur from the use of other regioisomeric alkylamines $\mathbf{5}$. Herein, we show that it is possible to obtain regioisomeric compounds 2, 3 and $\mathbf{4}$ from gold-catalyzed iso-Pictet-Spengler reactions (Scheme 1, eq. 2).

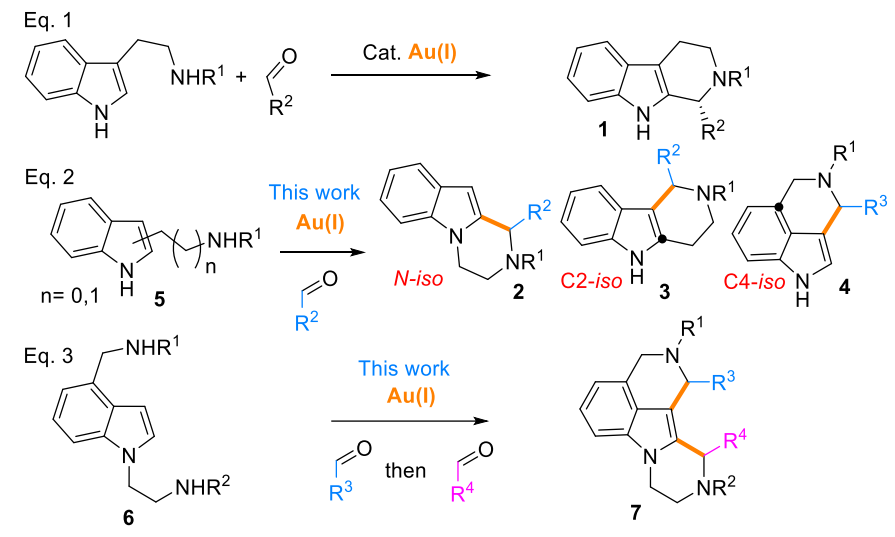

Scheme 1. Context of this work

For clarity purpose, we have defined the reactions as $\mathrm{N}$-iso-, C2-iso- and C4-iso-Pictet-Spengler reactions, depending on the connecting atom of the indole ring to the alkylamine chain. With a good knowledge of each of these different versions of the reaction, we could achieve a one-pot, selective process including successively a C4-iso and a $\mathrm{N}$-iso-Pictet-Spengler reaction for the synthesis of complex compounds 7 via the $\mathrm{C} 3$ then $\mathrm{C} 2$ ring-closures, with two different aldehydes (Scheme 1, eq. 3). An initial version of this work was deposited. ${ }^{16}$

\section{RESULTS AND DISCUSSION}

Our journey started with the study of the $\mathrm{N}$-iso-Pictet-Spengler reactions from $\mathrm{N}$-iso-tryptamine $\mathbf{5 a}$ and benzaldehyde $8 \mathrm{a}$, reacted in toluene in the presence of molecular sieves over a $40 \mathrm{~h}$ period (Table 1, see also Table S1 in the Supporting Information). In the absence of catalyst, no background reaction occurred (entry 1). Though counterintuitive, the reaction did not proceed either in the presence of an acidic catalyst, as previously reported ${ }^{7 \mathrm{~d}}$ for C3-unsubstituted $\mathrm{N}$-iso-tryptamines (entry 2). We next screened a series of three $\mathrm{Au}(\mathrm{I})$ catalysts (entries 3-5), for which only the Gagosz catalyst ${ }^{17}$ led to a moderate conversion (20\%, entry 4$)$. The solvent was replaced by DCM, allowing to reach a $69 \%$ conversion, further optimized to $74 \%$ after slight increase of the reaction temperature (entries 6-7). We have further checked that no background reaction occurs at this temperature in the absence of catalyst (entry 8). 
Table 1. Optimisation of the $\mathbf{N}$-iso Pictet-Spengler reaction

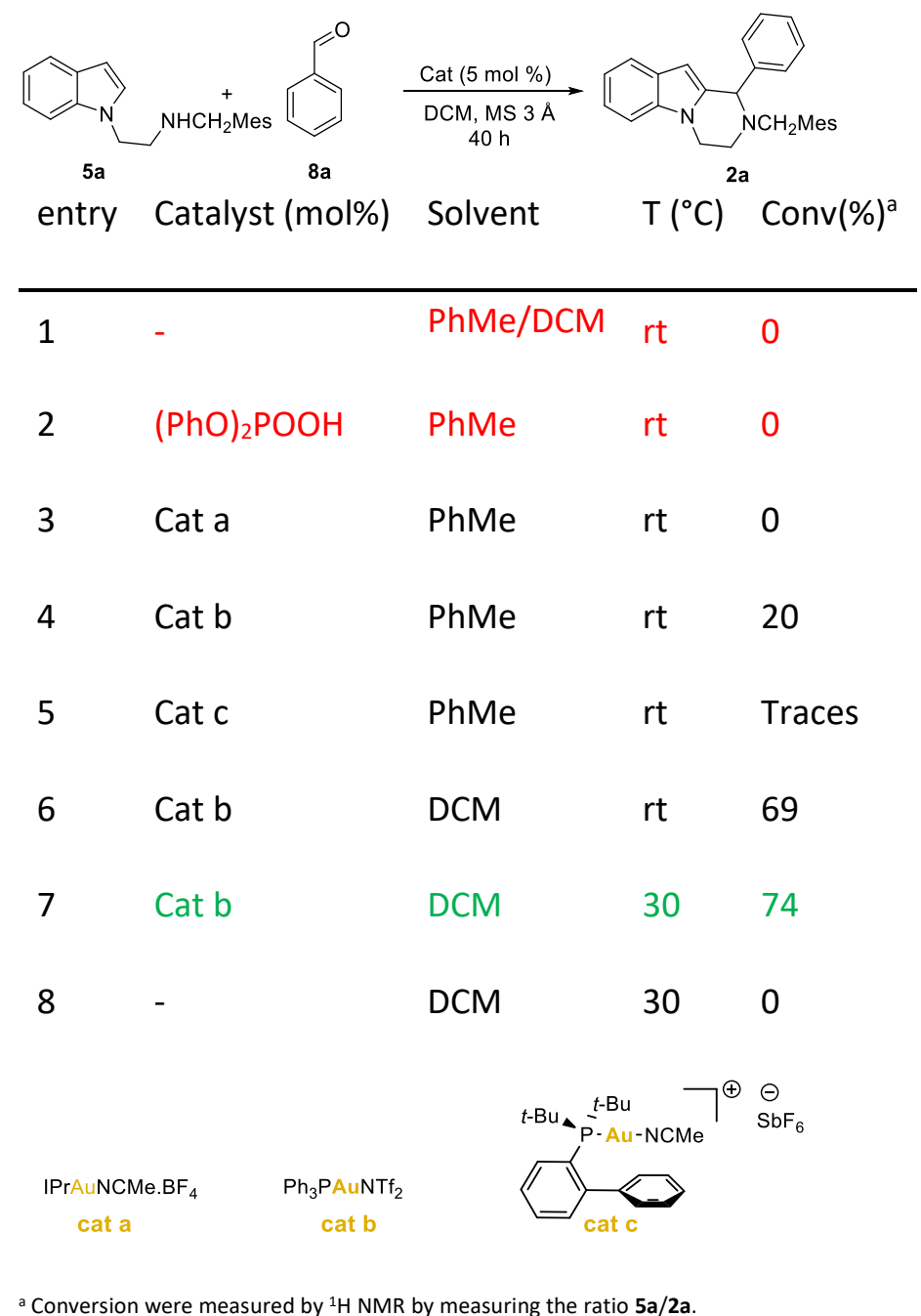

We next engaged a number of aromatic aldehydes in the reaction (Scheme 2, $\mathrm{N}$-iso Pictet-Spengler reaction). The reactions performed with benzaldehyde and $p$-bromobenzaldehyde led to $\mathbf{2 a}$ and $\mathbf{2 b}$ in $87 \%$ and $51 \%$ yields, respectively. A bromide group was tolerated at the meta and para positions, leading to compounds $\mathbf{2 c}$ and $\mathbf{2 d}$ in good yields. The reaction also proved compatible with aldehydes bearing electron-withdrawing $\mathrm{CF}_{3}$ groups at the para and meta positions. When $m$-methoxybenzaldehyde $\mathbf{8 g}$ was used, the corresponding product $\mathbf{2 g}$ was obtained in $59 \%$ yield. 4 - and 6-quinoline carbaldehydes then furnished compounds $\mathbf{2 h}$ and $\mathbf{2 i}$ in $87 \%$ and $93 \%$ yields, respectively. However, aliphatic aldehydes are not suitable substrates in this reaction, since 3-phenylpropanal did not lead to the expected compounds $\mathbf{2} \mathbf{j}$. An additional experiment performed in the presence of both benzaldehyde and 3-phenylpropanal ( 2 equiv. each) led only to the product $\mathbf{2 a}$, demonstrating the high chemoselectivity of this reaction towards arylaldehydes.

We switched to the gold-catalyzed C2-iso-Pictet-Spengler reaction, performed from isotryptamine $\mathbf{5 b}$ and a range of aldehydes (Scheme 2, C2-iso Pictet-Spengler reaction). Of note, this reaction is characterized by a strong background reaction when performed in the absence of catalyst (89\% conversion, see Table S2 in the supporting information). In view of potential future enantioselective applications, we sought for conditions ensuring a goldcatalyzed process. At $0{ }^{\circ} \mathrm{C}$ in the absence of catalyst, $23 \%$ conversion was obtained. However, this background reaction can be suppressed by decreasing the temperature to $-20^{\circ} \mathrm{C}$. At this temperature, the tetrahydro- $\gamma$-carboline $3 \mathbf{a}$ was obtained in $56 \%$ yield when the reaction was performed in the presence of complex $\mathbf{b}$ ( 5 mol\%), testifying for a solely $\mathrm{Au}(\mathrm{I})$-catalyzed process. Interestingly, the reaction showed a total selectivity between the nucleophilic C3 of the indole vs the potential reaction that could occur at the nitrogen atom. We screened a selection of functionalized arylaldehydes that could potentially result in reactivity issues if the reaction was acidcatalyzed and for each of these aldehydes, the absence of background reaction was checked at $\mathrm{rt}$ and $-20^{\circ} \mathrm{C}$ (see Figure S1 in the supporting information for details). In the presence of the gold(I) catalyst, compounds $\mathbf{3 b}$ and $\mathbf{3 c}$ bearing a 4-quinolynyl and 4-pyridyl chain, respectively, were obtained in good yields. Isophthalaldehyde led to 
the product $3 \mathbf{d}$ in $75 \%$ yield as a single product (no trace of doubly functionalized compound). Remarkably, despite a lower conversion and the need to react at $\mathrm{rt}$, the reaction also tolerated an aldehyde bearing a nitrone function, known to be activated by $\mathrm{Au}(\mathrm{I})$ complexes, ${ }^{18}$ leading to compound $3 \mathrm{e}$ in $47 \%$ yield. ${ }^{19}$ Comparatively, this kind of aldehyde would not be suitable in related acidic-catalyzed reactions, because of the activation of the highly electrophilic nitrone. All blank tests conducted with these aldehydes in the absence of catalyst show no conversion at $-20^{\circ} \mathrm{C}$ (and only little conversion at $\mathrm{rt}$, see the supporting information for details).

We further moved toward the introduction of the alkylamine chain at $\mathrm{C} 4$ atom of the indole ring and studied the reaction of tryptamine $\mathbf{5 c}$ with $p$-bromobenzalehyde in the presence (plain curves) of absence of catalyst (dashed curves) (Figure 2, see also Table S3 and Figure S2 in the Supporting Information). When the reaction was performed at $0{ }^{\circ} \mathrm{C}$, the reaction performed in the absence of catalyst barely showed conversion ( $<5 \%$ conversion), while the same reaction in the presence of 5 mol\% of cat $\mathbf{b}$ led to $50 \%$ conversion. At room temperature, we also observed a strong difference in the reaction kinetic, leading to $21 \%$ conversion in the absence of catalyst, while both $5 \mathrm{~mol} \%$ and $2 \mathrm{~mol} \%$ catalyst led to full conversions. The role of the gold catalyst is here again crucial to ensure full conversion over reasonable time, while conditions with solely Au(I)-catalyzed process can also be applied if required.

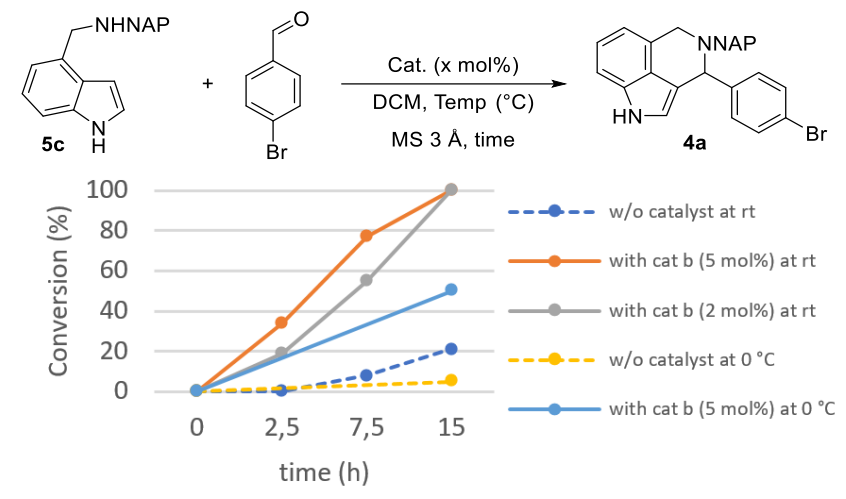

Figure 2. Reaction kinetic. Conversion were measured by ${ }^{1} \mathrm{H}$ NMR by measuring the ratio $5 \mathrm{c} / 4 \mathrm{a}$

Because of the attractively low 2 mol\% catalyst loading, we selected the conditions at room temperature to study the scope of this reaction (Scheme 2, C4-iso Pictet-Spengler reaction). The reaction of $\mathbf{5 c}$ with $p$-bromobenzaldehyde in DCM at room temperature in the presence of only 2 mol\% of Gagosz catalyst led to the corresponding 1,3,4,5-tetrahydropyrrolo[4,3,2-de]isoquinoline $4 a$ in $75 \%$ isolated yield. Benzaldehyde and $m$-trifluoromethylbenzaldehyde also led to excellent yields in $\mathbf{4 b , c}$. A fluorine group at the ortho position was well tolerated, while a methoxy group at the para position led to a decrease of the reactivity ( $4 \mathrm{e}, 38 \%$ yield). The phthalaldehyde led to compound $\mathbf{4 f}$ in $\mathbf{4 6 \%}$ yield, this time accompanied by the dimeric product (ratio $\mathbf{4 f} /$ dimer: $10 / 4$ ). A boronate ester at the para position was tolerated, leading to $\mathbf{4 g}$ in $53 \%$ yield, opening opportunities for subsequent crosscoupling functionnalizations. $\mathbf{4 h}$, bearing a quinolyl moiety was formed in $71 \%$ yield. Interestingly, aliphatic aldehydes were well converted to compounds $4 \mathbf{i}-\mathbf{k}$ in moderate to good yields, while the same reactions performed in the absence of catalyst resulted in no conversions (see Table S4 and Figure S3 in the supporting information for details). To date, this is the first time that we observed conversion in the course of any version of the four different $\mathrm{Au}(\mathrm{I})$-catalyzed (iso)Pictet-Spengler reactions that we studied with aliphatic aldehydes.

We next considered the possibility that an indole ring functionalized with two alkylamine chains at the $\mathrm{N}$ - and $\mathrm{C} 4$ position could undergo $\mathrm{C} 2-\mathrm{C} 3$ difunctionalization via a $\mathrm{C} 4$-iso and a $\mathrm{N}$-iso-Pictet-Spengler cyclization cascade. For this purpose, we designed the diamine $\mathbf{5 d}$, keeping the same groups on the nitrogen atoms and slightly increased the temperature to $40^{\circ} \mathrm{C}$ to ensure maximum conversion.

Indeed, gold-catalyzed reaction of $\mathbf{5 d}$ with benzaldehyde at $40{ }^{\circ} \mathrm{C}$ led to tetracyclic $2,3,8,9,10,11$-hexahydro- $1 \mathrm{H}$ pyrazino[1',2':1,5]pyrrolo[4,3,2-de]isoquinoline $9 \mathrm{a}$ in $68 \%$ yield and a 70/30 diatereomeric ratio, in favor of the anti diastereoisomer (Scheme 3a, see also Table S5 in the Supporting Information for optimization). ${ }^{20}$ To the best of our knowledge, this scaffold has never been reported. When the meta-trifluoromethylbenzaldehyde was used, compound $\mathbf{9 b}$ was obtained in $58 \%$ yield, with the diasteromeric ratio in favor of the syn isomer. 4 -Quinoline carboxaldehyde led to the amine $9 \mathrm{c}$ in $88 \%$ yield and full anti-diastereoselectivity. We next reasoned that the C4- 
iso Pictet-Spengler reaction, operating with lower catalyst loading and temperature, should occur faster than the $\mathrm{N}$-iso reaction, requiring higher catalyst loading and temperature. Indeed, when $\mathbf{5} \mathbf{d}$ was engaged in the reaction with 1 equivalent of $p$-bromobenzaldehyde, the C4-iso Pictet-Spengler product 10a was selectively obtained at room temperature in $71 \%$ yield with 10 mol\% cat b (Scheme $3 b$, eq. 1 ).

Control experiments revealed that: 1 ) no background reaction occurs in the absence of $A u(I)$ catalyst on substrate $\mathbf{5 d}$ and 2 ) it is necessary to increase the catalyst loading to ensure a good conversion in this step. Compound 10a was then engaged in the $\mathrm{N}$-iso Pictet-Spengler reaction with benzaldehyde that led to $11 \mathrm{a}$ in $70 \%$ yield as a mixture of diastereomers (Scheme 3b, eq. 2, dr 25/75).

We then developed the one-pot formation of compounds $\mathbf{1 1}$ by sequential addition of two different aldehydes from $\mathbf{5 d}$ in the presence of $10 \mathrm{~mol} \%$ of the gold complex $\mathbf{b}$ as catalyst (Scheme $3 \mathrm{c}$ ). After $15 \mathrm{~h}$ of reaction in the presence of $p$-bromobenzaldehyde (1 equiv) at room temperature, benzaldehyde was added to the reaction mixture and further $24 \mathrm{~h}$ reaction led to compound 11a in $72 \%$ yield ( $\mathrm{dr} 35 / 65$ ) in favor of the syn isomer.

The same protocol was applied to another couple of aromatic aldehydes, leading to compound $\mathbf{1 1 b}$ in good yields and good diastereoselectivity ( $\mathrm{dr}$ 20/80). Gratifyingly, we obtained the X-ray structure of the syn-diastereomer of 11b (CCDC 2046851). Beyond confirming its structure, it was helpful to further identify all other diastereomers of the series of compounds $\mathbf{9}$ and $\mathbf{1 1}$. When 4-quinolinecarboxaldehyde was used in the first C4-iso-Pictet-Spengler step (requiring a higher $40{ }^{\circ} \mathrm{C}$ temperature), followed by benzaldehyde for the $\mathrm{N}$-iso step, compound $11 \mathrm{c}$ was obtained in $71 \%$ yield and diastereoselectivity in favor of the anti-isomer (CCDC 2046871). The diastereoselectivity of these reactions may be the result of electronic effects. Indeed, the X-ray structure of the syn-11b shows a pretty good superposition of the phenyl and the trifluoromethylphenyl ring with a distance between the two aryl planes of ca. 3.30 Ả. As reviewed by Iverson, interactions between electron-poor/electron rich aryls may be referred to the favored "aromatic donor- acceptor interactions" or polar/ $\pi$ model. ${ }^{21}$ This would explain the general trend to lead to the syn-diastereomer with electron-poor aryl (9b, 11a, 11b) while destabilizing interactions operating with electron-rich aryl rings couples (phenyl, quinoline and their combination) lead mainly to the anti-isomer (9a, 9c, 11c).

Gratifyingly, no need for an addition batch of catalyst was required for the application of this protocol. The formation of these compounds, as the result of two successive iso-Pictet-Spengler reactions with different aldehydes, opens avenues for the synthesis of highly functionalized and complex compounds. This strategy potentially offers unique opportunities for the exploration of chemical space in biological studies. 

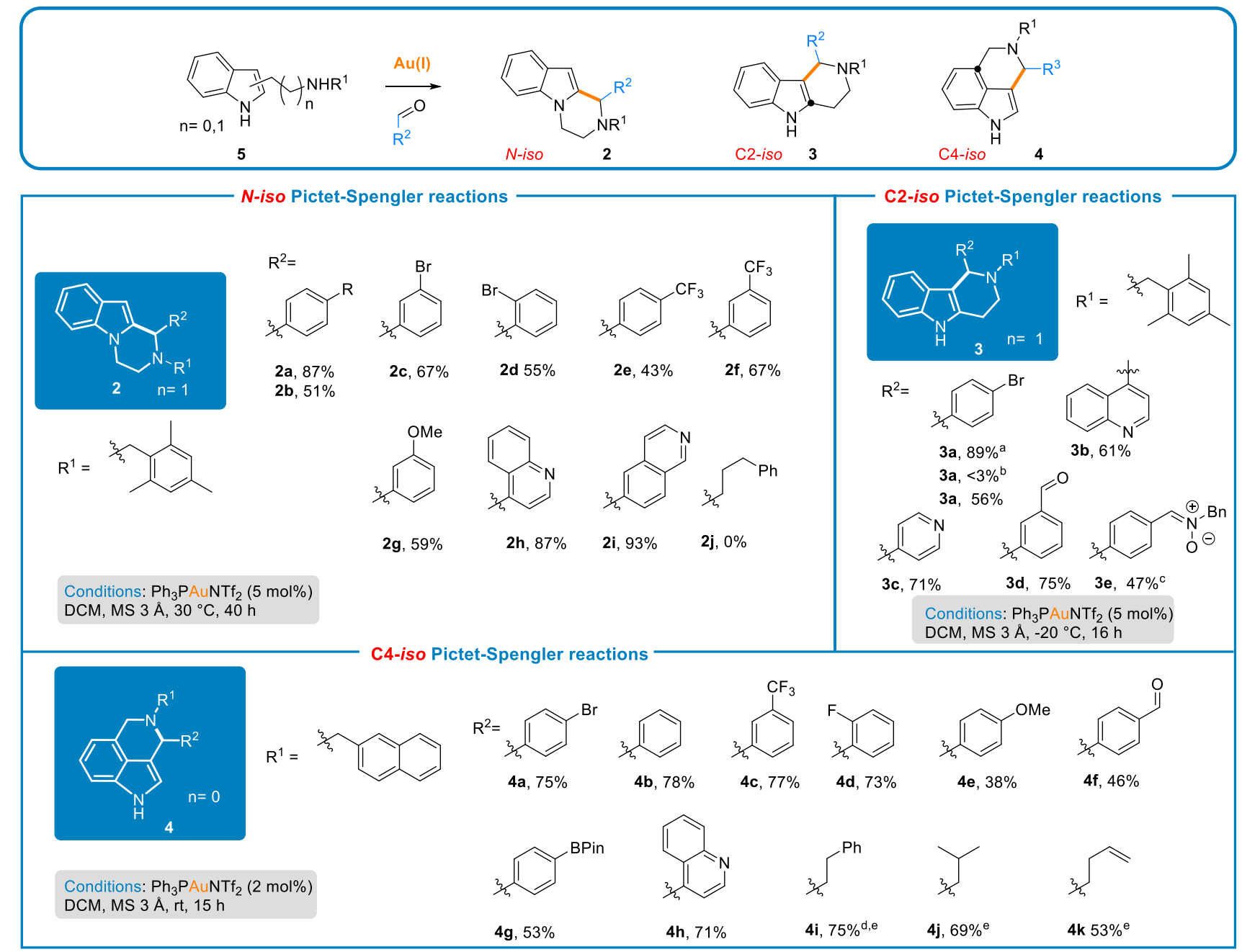

a Performed at room temperature in the absence of catalyst. ${ }^{\mathrm{b}}$ Performed at $-20{ }^{\circ} \mathrm{C}$ in the absence of catalyst. ${ }^{\mathrm{c}} \mathrm{Performed}$ at $\mathrm{rt}$. ${ }^{\mathrm{d}} \mathrm{Performed}$ with 5 mol\% of $\mathrm{Ph}{ }_{3} \mathrm{PAuNTf}{ }_{2}$. e Conversion is $0 \%$ in the absence of catalyst.

\section{Scheme 2. Scope of the different versions of the iso-Pictet-Spengler reactions studied}




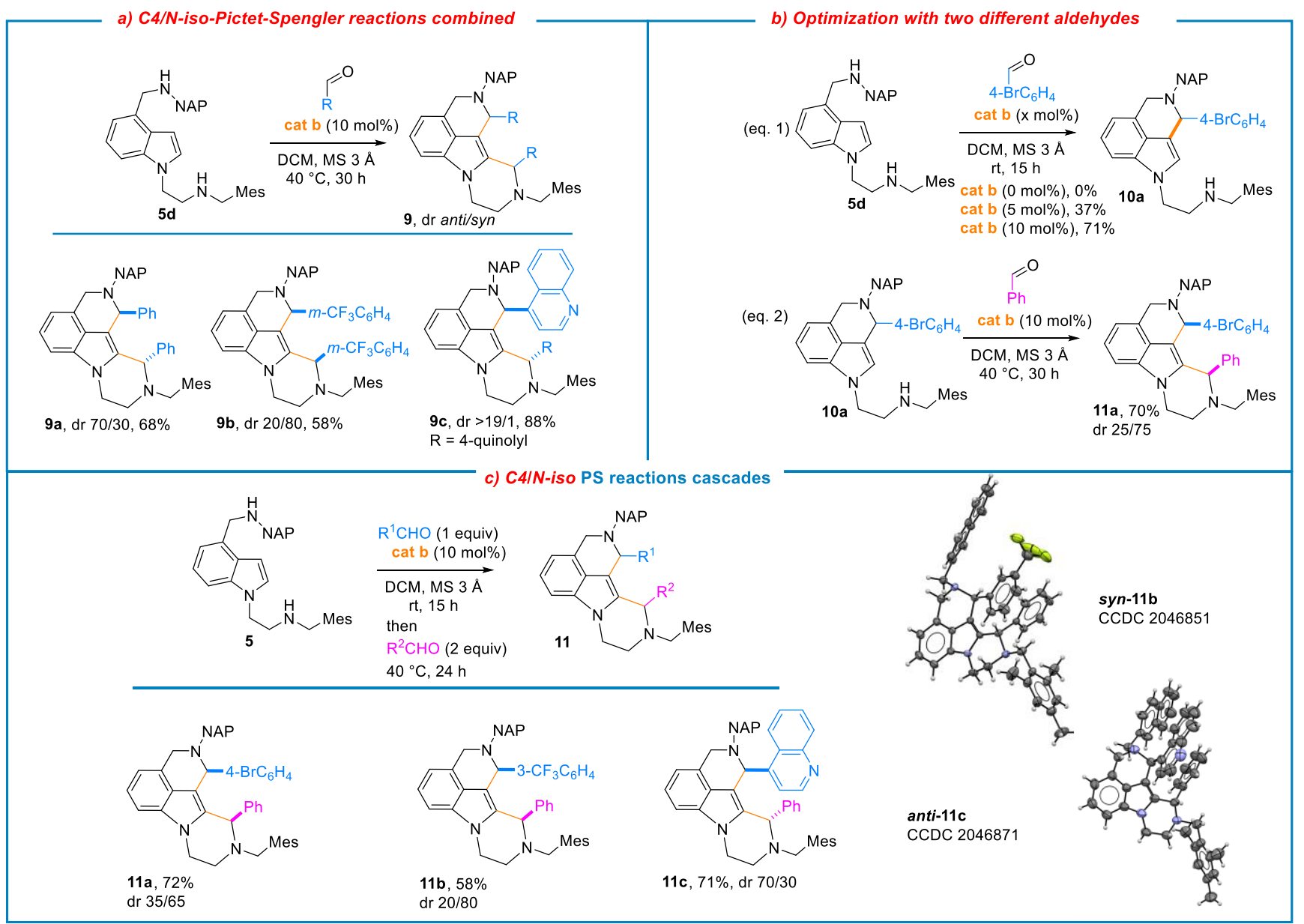

Scheme 3. Selective bis-functionnalization (the relative stereochemistry indicated is that of the major diastereomer)

The mechanistic hypothesis for these reactions relies on our previous gold-catalyzed "classical" PictetSpengler reactions ${ }^{14 a}$ and is illustrated below with the $\mathrm{N}$-iso-version of the reaction (Scheme 4), with the following steps. (i) The spontaneous addition of the amine to the aldehyde leads to an hemiaminal (this step being potentially catalyzed by the $\mathrm{Au}(\mathrm{I})$ complex). (ii) The coordination of the indole ring leads to the $\eta^{2}$ and $\eta^{1}$-gold complexes $\mathbf{A}$ and $\mathbf{B}$. (iii) The conversion of the latter to an iminium via an intramolecular abstraction of a proton and release of water generates $\mathbf{C}^{22}$ (iv) the nucleophilic addition to the iminium via $\mathrm{C} 2$ forms complexO D. (v) The elimination of a proton via $\mathbf{E}$ and protodeauration then leads to the product $\mathbf{2}$ and the regeneration of the cationic $A u(I)$ complex. Similar mechanisms can be involved for the $\mathrm{C2}$ - and C4-isoPictet-Spengler reactions (see Schemes S1, S2 and S3 in the supporting Information).

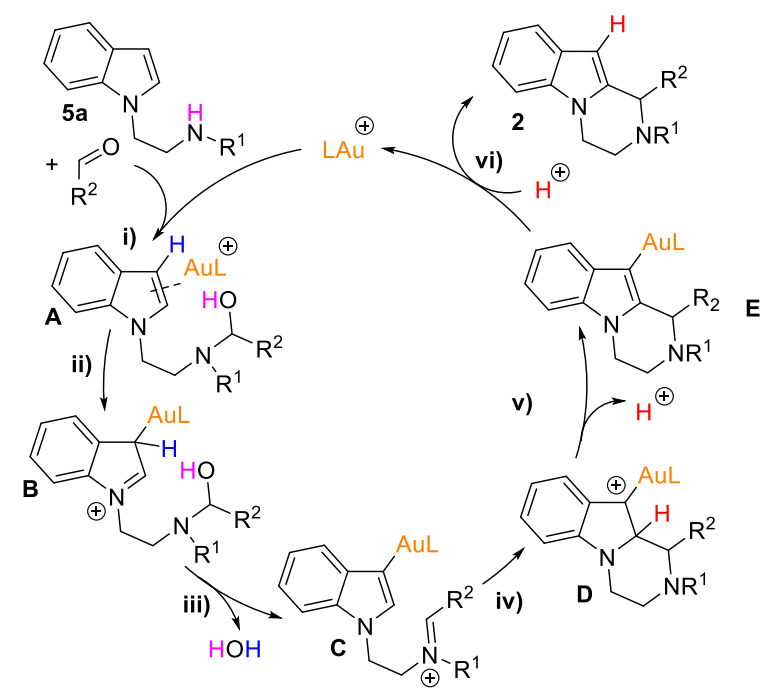

Scheme 4. Mechanistic pathway in $\mathrm{N}$-iso-Pictet-Spengler reactions

\section{CONCLUSION}

To conclude, we have developed $\mathrm{Au}(\mathrm{I})$-catalyzed isoPictet-Spengler reactions by introduction of the alkylamine chain around all the different positions of the 
indole ring allowing a trapping of the in situ generated iminium ion by either the $\mathrm{C} 2$ or $\mathrm{C} 3$ atom. This led to the isolation of numerous heterocyclic scaffolds. We have showed the high chemoselectivity enabled by $\mathrm{Au}(\mathrm{I})$ catalyzed processes in these reactions, in particular by design of the in situ sequential cascade of C4and $\mathrm{N}$-iso-Pictet-Spengler reactions leading to highly complex polycyclic indolic arrangements. We are currently studying the enantioselective gold-catalyzed version of these reactions.

\section{EXPERIMENTAL SECTION}

Reactions were performed using oven-dried glassware under an argon atmosphere. All separations were carried out under flashchromatographic conditions on silica gel (prepacked column, 230400 mesh) at medium pressure (20 psi) with the use of a CombiFlash Companion. Reactions were monitored by thin-layer chromatography on Merck silica gel plates (60 F254 aluminum sheets), which were rendered visible by ultraviolet and spraying with vanillin (15\%) + sulfuric acid (2.5\%) in EtOH followed by heating. Reagent-grade chemicals were obtained from diverse commercial suppliers (Sigma-Aldrich, Acros Organics, Fluorochem, $\mathrm{TCl}$ and Alfa-Aesar) and were used as received. ${ }^{1} \mathbf{H}$ NMR $(500$ or $300 \mathrm{MHz}$ ) and ${ }^{13} \mathrm{C}$ NMR (125 or $75 \mathrm{MHz}$ ) spectra were recorded on Brücker Avance spectrometers at $298 \mathrm{~K}$ unless otherwise stated. Chemical shifts are given in ppm ( $\delta)$ and are referenced to the internal solvent signal or to TMS used as an internal standard. Multiplicities are declared as follow: $s$ (singlet), brs (broad singlet), $d$ (doublet), $t$ (triplet), $q$ (quadruplet), $d d$ (doublet of doublet), $d d d$ (doublet of doublet of doublet), $d t$ (doublet of triplet), $m$ (multiplet), $A B=A B$ quartet, $A B X=A B X$ system. Coupling constants $J$ are given in $\mathrm{Hz}$. Carbon multiplicities were determined by DEPT135 experiment. Diagnostic correlations were obtained by two-dimensional COSY, HSQC and NOESY experiments. Infrared spectra (IR) were recorded on a Perkin-Elmer FT-IR system using diamond window Dura SamplIR II and the data are reported in reciprocal centimeters (cm-1). High-resolution mass spectra (HRMS) were recorded using a Micromass LCT Premier XE instrument (Waters) and were determined by electrospray ionization (ESI, TOF analyzer).

All reactions were accordingly performed using purified aldehydes. Aldehydes were purified by washing a solution of the aldehyde in $\mathrm{Et}_{2} \mathrm{O}$ by $\mathrm{NaOH}\left(2 \mathrm{M}\right.$ in $\mathrm{H}_{2} \mathrm{O}$ ), followed by drying on $\mathrm{MgSO}_{4}$ and filtration of the resulting solution on a short pad of silica gel, followed by concentration under vacuum. In addition, tryptamines were dissolved in EtOAc and washed by $\mathrm{NaOH}\left(2 \mathrm{M}\right.$ in $\mathrm{H}_{2} \mathrm{O}$ ) on a regular basis, to avoid undesired catalysis from potential protonated amines. Finally, molecular sieves must be powdered and activated for two hours under vacuum at $200{ }^{\circ} \mathrm{C}$ before use. All reactions requiring heating were heated with an oil bath.

N-Iso Pictet-Spengler reaction

Synthesis of 2 -(1H-indol-1-yl)- $N$-(2,4,6-trimethylbenzyl)ethan-1amine 5 a

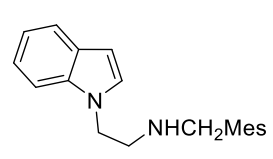

2-(1H-indol-1-yl)ethan-1-amine (prepared according to the procedure described by Verma in $84 \%$ yield) ${ }^{7 e}(1.5 \mathrm{~g}, 9.4 \mathrm{mmol}$, 1.00 equiv.), 2,4,6-trimethylbenzaldehyde (1,32 g, $8.9 \mathrm{mmol}, 0.95$ equiv.) were stirred in methanol $(40 \mathrm{~mL})$ under an argon atmosphere for 36 hours. Then, the reaction media was cooled to $0{ }^{\circ} \mathrm{C}$ before adding
$\mathrm{NaBH}_{4}(2 * 262 \mathrm{mg}, 13.8 \mathrm{mmol}, 1.80$ equiv.), then the mixture was allowed to reach room temperature. After 1 hour stirring, the volatiles were removed and the crude mixture was next diluted in ethyl acetate and water. After the phases were separated, the aqueous phase was extracted twice by ethyl acetate then the combined organic phases were dried over $\mathrm{MgSO}_{4}$ and evaporated under vacuum. The desired product $\mathbf{5} \mathbf{a}$ was obtained after column chromatography on silica gel (gradient from 20 to $100 \%$ Heptane:EtOAc) as a greenish oil (1.37 g, $4.7 \mathrm{mmol}, 50 \%)$. IR (neat) $\mathbf{v}_{\max }=2915,1612,1511,1462,1313,1113,1012 \mathrm{~cm}^{-1} .{ }^{1} \mathbf{H}$ NMR $\left(\mathrm{CDCl}_{3}, 500 \mathrm{MHz}\right) \delta=7.67(\mathrm{~d}, J=7.6 \mathrm{~Hz}, 1 \mathrm{H}), 7.42(\mathrm{~d}, J=8.0 \mathrm{~Hz}$, $1 \mathrm{H}), 7.24$ (td, $J=7.3,0.7 \mathrm{~Hz}, 1 \mathrm{H}), 7.18-7.11(\mathrm{~m}, 2 \mathrm{H}), 6.84(\mathrm{~s}, 2 \mathrm{H})$, $6.52(\mathrm{~d}, J=3.1 \mathrm{~Hz}, 1 \mathrm{H}), 4.29(\mathrm{~d}, J=6.3 \mathrm{~Hz}, 2 \mathrm{H}), 3.76(\mathrm{~s}, 2 \mathrm{H}), 3.15(\mathrm{t}$, $J=6.3 \mathrm{~Hz}, 1 \mathrm{H}), 2.29(\mathrm{~s}, 6 \mathrm{H}), 2.27(\mathrm{~s}, 3 \mathrm{H}) .{ }^{13} \mathrm{C}\left\{{ }^{1} \mathrm{H}\right\} \mathrm{NMR}\left(\mathrm{CDCl}_{3}, 75\right.$ $\mathrm{MHz}) \delta=137.1\left(\mathrm{C}_{\mathrm{q}}\right), 136.8\left(\mathrm{C}_{\mathrm{q}}\right), 136.2\left(\mathrm{C}_{\mathrm{q}}\right), 133.2\left(\mathrm{C}_{\mathrm{q}}\right), 129.2(\mathrm{CH})$, $128.9\left(\mathrm{C}_{\mathrm{q}}\right), 128.2(\mathrm{CH}), 121.7(\mathrm{CH}), 121.2(\mathrm{CH}), 119.5(\mathrm{CH}), 109.5$ (CH), $101.5(\mathrm{CH}), 49.5\left(\mathrm{CH}_{2}\right), 47.7\left(\mathrm{CH}_{2}\right), 46.8\left(\mathrm{CH}_{2}\right), 21.0\left(\mathrm{CH}_{3}\right), 19.7$ $\left(\mathrm{CH}_{3}\right)$. HRMS (ESI): $\mathrm{m} / \mathrm{z}$ : calc for $\mathrm{C}_{20} \mathrm{H}_{25} \mathrm{~N}_{2}{ }^{+} 293.2017[\mathrm{M}+\mathrm{H}]^{+}$, found 293.2007.

General procedure 1 for the synthesis of tetrahydropyrazino[1,2-a]indoles 2: A mixture of $\mathrm{N}$-iso tryptamine 5a $(0.15$ $\mathrm{mmol})$, Cat b ( $5 \mathrm{~mol} \%, 0.0075 \mathrm{mmol}, 5.3 \mathrm{mg}$ ) and $3 \AA$ A molecular sieves (150 mg for $0.15 \mathrm{mmol}$ of $\mathbf{5 a}$, powdered) in dichloromethane $(1.5 \mathrm{~mL}$ for $0.15 \mathrm{mmol}$ of $\mathbf{5 a}$ ) was stirred for $5 \mathrm{~min}$ at room temperature under an argon atmosphere. Subsequently, aldehyde ( 2.0 equiv., $0.30 \mathrm{mmol}$ ) was added and the mixture stirred for $40 \mathrm{~h}$ at $30^{\circ} \mathrm{C}$. For practical reasons, the excess of aldehyde was reduced at the end of the reaction by mean of $\mathrm{NaBH}_{4}$ to facilitate the purification. The reaction mixture was then cooled to $0{ }^{\circ} \mathrm{C}$ and methanol ( $1.5 \mathrm{~mL}$ for $0.15 \mathrm{mmol}$ of $5 \mathrm{a}$ ) alongside sodium borohydride ( 2.0 equiv.) was added to the reaction mixture. It was then allowed to reach room temperature and stirred for 1 hour. Then it was filtered under celite and silica was added. After evaporation of the volatiles, the silica mixture was purified by chromatography under silica gel to give the desired product $\mathbf{2}$.

1-phenyl-2-(2,4,6-trimethylbenzyl)-1,2,3,4-tetrahydropyrazino[1,2-a]indole $2 \mathrm{a}$

Compound $2 \mathrm{a}$ was synthesized fol-

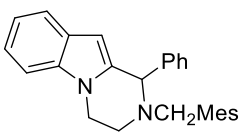
lowing the General procedure 1 using tryptamine $5 a$ ( $44 \mathrm{mg}, 0.15 \mathrm{mmol}, 1.0$ equiv.), benzaldehyde (32 $\mathrm{mg}, 0.30 \mathrm{mmol}, 2.0$ equiv.), Cat b $(5.3 \mathrm{mg}, 0.0075 \mathrm{mmol})$, in DCM (1.5 mL). The desired product 2 a was obtained after column chromatography on silica gel (gradient from 0 to $50 \%$ Heptane:EtOAc) as a green amorphous solid (49 mg, $0.13 \mathrm{mmol}, 87 \%$ ). IR (neat) $\boldsymbol{v}_{\max }=2919,1612$, 1451, 1375, 1312, 1265, 1228, 1148, $1074 \mathrm{~cm}^{-1} .{ }^{1} \mathrm{H}$ NMR $\left(\mathrm{CDCl}_{3}\right.$, $500 \mathrm{MHz}) \delta=7.52(\mathrm{~d}, J=7.8 \mathrm{~Hz}, 1 \mathrm{H}), 7.50-7.46(\mathrm{~m}, 2 \mathrm{H}), 7.42-7.36$ (m, 3H), $7.31(\mathrm{~d}, J=7.8 \mathrm{~Hz}, 1 \mathrm{H}), 7.20(\mathrm{td}, J=7.0,0.6 \mathrm{~Hz}, 1 \mathrm{H}), 7.12$ $(\mathrm{td}, J=7.0,0.6 \mathrm{~Hz}, 1 \mathrm{H}), 6.88(\mathrm{~s}, 2 \mathrm{H}), 5.82(\mathrm{~s}, 1 \mathrm{H}), 4.69(\mathrm{~s}, 1 \mathrm{H}), 4.16$ (dt, $J=11.3,3.6 \mathrm{~Hz}, 1 \mathrm{H}), 3.98(\mathrm{dt}, J=10.4,4.6 \mathrm{~Hz}, 1 \mathrm{H}), 3.71(\mathrm{~d}, J=$ $12.5 \mathrm{~Hz}, 1 \mathrm{H}), 3.39(\mathrm{~d}, J=12.5 \mathrm{~Hz}, 1 \mathrm{H}), 3.21(\mathrm{dt}, J=12.1,4.1 \mathrm{~Hz}, 1 \mathrm{H})$, $2.81(\mathrm{td}, J=12.1,4.0 \mathrm{~Hz}, 1 \mathrm{H}), 2.31(\mathrm{~s}, 9 \mathrm{H}) .{ }^{13} \mathrm{C}\left\{{ }^{1} \mathrm{H}\right\} \mathrm{NMR}\left(\mathrm{CDCl}_{3}, 75\right.$ $\mathrm{MHz}) \delta=141.7\left(\mathrm{C}_{\mathrm{q}}\right), 139.1\left(\mathrm{C}_{\mathrm{q}}\right), 138.6\left(\mathrm{C}_{\mathrm{q}}\right), 136.8\left(\mathrm{C}_{\mathrm{q}}\right), 136.0\left(\mathrm{C}_{\mathrm{q}}\right)$, $131.5\left(\mathrm{C}_{\mathrm{q}}\right), 129.9(\mathrm{CH}), 129.3(\mathrm{CH}), 128.4\left(\mathrm{C}_{\mathrm{q}}\right), 128.2(\mathrm{CH}), 128.1$ $(\mathrm{CH}), 120.9(\mathrm{CH}), 120.4(\mathrm{CH}), 120.0(\mathrm{CH}), 108.9(\mathrm{CH}), 100.2\left(\mathrm{C}_{\mathrm{q}}\right)$, $99.7(\mathrm{CH}), 67.6(\mathrm{CH}), 52.4\left(\mathrm{CH}_{2}\right), 47.0\left(\mathrm{CH}_{2}\right), 42.0\left(\mathrm{CH}_{2}\right), 21.1\left(\mathrm{CH}_{3}\right)$, $20.7\left(\mathrm{CH}_{3}\right)$. HRMS (ESI): $\mathrm{m} / \mathrm{z}$ : calc for $\mathrm{C}_{27} \mathrm{H}_{29} \mathrm{~N}_{2}{ }^{+} 381.2331[\mathrm{M}+\mathrm{H}]^{+}$, found 381.2335 
1-(4-bromophenyl)-2-(2,4,6-trimethylbenzyl)-1,2,3,4-tetrahydropyrazino $[1,2-a]$ indole $\mathbf{2 b}$

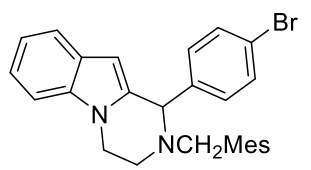

Compound $\mathbf{2 b}$ was synthesized following the General procedure 1 using tryptamine 5a $(44 \mathrm{mg}, 0.15 \mathrm{mmol}, 1.0$ equiv.), 4-bromobenzaldehyde $(55 \mathrm{mg}$, $0.30 \mathrm{mmol}, 2.0$ equiv.), Cat b $(5.3 \mathrm{mg}$, $0.0075 \mathrm{mmol})$, in DCM $(1.5 \mathrm{~mL})$. The desired product $\mathbf{2} \mathbf{b}$ was obtained after column chromatography on silica gel (gradient from 0 to $50 \%$ Heptane:EtOAc) as a green amorphous solid ( $35 \mathrm{mg}, 0.076$ mmol, $51 \%)$. IR (neat) $\mathbf{v}_{\max }=2970,1737,1451,1366,1217$, $1011 \mathrm{~cm}^{-1} .{ }^{1} \mathbf{H}$ NMR $\left(\mathrm{CDCl}_{3}, 500 \mathrm{MHz}\right) \delta=7.42-7.36(\mathrm{~m}, 3 \mathrm{H}), 7.23$ (d, $J=7.6 \mathrm{~Hz}, 1 \mathrm{H}), 7.18(\mathrm{~d}, J=8.1 \mathrm{~Hz}, 1 \mathrm{H}), 7.08(\mathrm{t}, J=7.5 \mathrm{~Hz}, 1 \mathrm{H})$, $7.00(\mathrm{t}, J=7.4 \mathrm{~Hz}, 1 \mathrm{H}), 6.75(\mathrm{~s}, 2 \mathrm{H}), 5.69(\mathrm{~s}, 1 \mathrm{H}), 4.55(\mathrm{~s}, 1 \mathrm{H}), 4.07$ $4.01(\mathrm{~m}, 1 \mathrm{H}), 3.90-3.81(\mathrm{~m}, 1 \mathrm{H}), 3.57(\mathrm{~d}, J=12.5 \mathrm{~Hz}, 1 \mathrm{H}), 3.28$ (d, J $=12.5 \mathrm{~Hz}, 1 \mathrm{H}), 3.08(\mathrm{~d}, J=12.0 \mathrm{~Hz}, 1 \mathrm{H}), 2.81(\mathrm{t}, J=9 \mathrm{~Hz}, 1 \mathrm{H}), 2.18$ $(\mathrm{s}, 3 \mathrm{H}), 2.17(\mathrm{~s}, 6 \mathrm{H}) .{ }^{13} \mathrm{C}\left\{{ }^{1} \mathrm{H}\right\} \mathrm{NMR}\left(\mathrm{CDCl}_{3}, 75 \mathrm{MHz}\right) \delta=140.9\left(\mathrm{C}_{\mathrm{q}}\right)$, $138.5\left(\mathrm{C}_{\mathrm{q}}\right), 138.2\left(\mathrm{C}_{\mathrm{q}}\right), 137.0\left(\mathrm{C}_{\mathrm{q}}\right), 136.0\left(\mathrm{C}_{\mathrm{q}}\right), 131.5(\mathrm{CH}), 131.4(\mathrm{CH})$, $131.2(\mathrm{Cq}), 129.3(\mathrm{CH}), 128.3\left(\mathrm{C}_{\mathrm{q}}\right), 122.0(\mathrm{Cq}), 121.2(\mathrm{CH}), 120.5$ $(\mathrm{CH}), 120.1(\mathrm{CH}), 110.7\left(\mathrm{C}_{\mathrm{q}}\right), 108.9\left(\mathrm{C}_{\mathrm{q}}\right), 99.9(\mathrm{CH}), 66.6(\mathrm{CH}), 52.4$ $\left(\mathrm{CH}_{2}\right), 46.8\left(\mathrm{CH}_{2}\right), 41.8\left(\mathrm{CH}_{2}\right), 21.1\left(\mathrm{CH}_{3}\right), 20.7\left(\mathrm{CH}_{3}\right)$. HRMS (ESI): $\mathrm{m} / \mathrm{z}$ : calc for $\mathrm{C}_{7} \mathrm{H}_{28} \mathrm{~N}_{2} \mathrm{Br}^{+} 459.1436[\mathrm{M}+\mathrm{H}]^{+}$, found 459.1421

1-(3-bromophenyl)-2-(2,4,6-trimethylbenzyl)-1,2,3,4-tetrahydropyrazino[1,2-a]indole $\mathbf{2 c}$

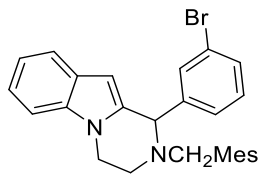

Compound 2c was synthesized following the General procedure 1 using tryptamine 5 a ( $44 \mathrm{mg}, 0.15 \mathrm{mmol}, 1.0$ equiv.), 3-bromobenzaldehyde $(55 \mathrm{mg}, 0.30 \mathrm{mmol}$, 2.0 equiv.), Cat b (5.3 $\mathrm{mg}, 0.0075 \mathrm{mmol})$, in DCM (1.5 mL). The desired product $\mathbf{2 c}$ was obtained after column chromatography on silica gel (gradient from 0 to $50 \% \mathrm{Hep}$ tane:EtOAc) as a green amorphous solid ( $46 \mathrm{mg}, 0.10 \mathrm{mmol}, 67 \%$ ). IR (neat) $v_{\max }=2922,2853,1739,1571,1452,1375,1319,1217$, 1149, $1070 \mathrm{~cm}^{-1} .{ }^{1} \mathrm{H} \mathrm{NMR}\left(\mathrm{CDCl}_{3}, 500 \mathrm{MHz}\right) \delta=7.61(\mathrm{~s}, 1 \mathrm{H}), 7.50$ (d, $J=7.7 \mathrm{~Hz}, 1 \mathrm{H}), 7.47(\mathrm{~d}, J=7.9 \mathrm{~Hz}, 1 \mathrm{H}), 7.37(\mathrm{~d}, J=7.7 \mathrm{~Hz}, 1 \mathrm{H})$, 7.29 (d, $J=8.1 \mathrm{~Hz}, 1 \mathrm{H}), 7.22(\mathrm{t}, J=7.9 \mathrm{~Hz}, 1 \mathrm{H}), 7.18(\mathrm{t}, J=7.9 \mathrm{~Hz}$, $1 \mathrm{H}), 7.10(\mathrm{t}, J=7.9 \mathrm{~Hz}, 1 \mathrm{H}), 6.85(\mathrm{~s}, 2 \mathrm{H}), 5.82(\mathrm{~s}, 1 \mathrm{H}), 4.64(\mathrm{~s}, 1 \mathrm{H})$, 4.15-4.10 (m, 1H), $3.94(\mathrm{td}, J=10.6,4.4 \mathrm{~Hz}, 1 \mathrm{H}), 3.66(\mathrm{~d}, J=12.5$ $\mathrm{Hz}, 1 \mathrm{H}), 3.39(\mathrm{~d}, J=12.5 \mathrm{~Hz}, 1 \mathrm{H}), 3.18(\mathrm{dt}, J=11.9,3.8 \mathrm{~Hz}, 1 \mathrm{H}), 2.79$ $(\mathrm{td}, J=11.0,4.0 \mathrm{~Hz}, 1 \mathrm{H}), 2.27(\mathrm{~s}, 9 \mathrm{H}) .{ }^{13} \mathrm{C}\left\{{ }^{1} \mathrm{H}\right\} \mathrm{NMR}\left(\mathrm{CDCl}_{3}, 75 \mathrm{MHz}\right)$ $\delta=144.2\left(C_{q}\right), 138.6\left(C_{q}\right), 138.0\left(C_{q}\right), 137.0\left(C_{q}\right), 136.0\left(C_{q}\right), 132.7$ $(\mathrm{CH}), 131.2(\mathrm{CH}), 131.1\left(\mathrm{C}_{\mathrm{q}}\right), 129.8(\mathrm{CH}), 129.4(\mathrm{CH}), 128.3(\mathrm{CH})$, $128.3\left(\mathrm{C}_{\mathrm{q}}\right), 122.4\left(\mathrm{C}_{\mathrm{q}}\right), 121.2(\mathrm{CH}), 120.5(\mathrm{CH}), 120.1(\mathrm{CH}), 109.0$ $(\mathrm{CH}), 100.2\left(\mathrm{C}_{\mathrm{q}}\right), 99.9(\mathrm{CH}), 66.7(\mathrm{CH}), 52.5\left(\mathrm{CH}_{2}\right), 46.8\left(\mathrm{CH}_{2}\right), 41.8$ $\left(\mathrm{CH}_{2}\right), 21.1\left(\mathrm{CH}_{3}\right), 20.6\left(\mathrm{CH}_{3}\right)$. HRMS (ESI): $\mathrm{m} / \mathrm{z}$ : calc for $\mathrm{C}_{27} \mathrm{H}_{28} \mathrm{~N}_{2} \mathrm{Br}^{+}$ $459.1436[\mathrm{M}+\mathrm{H}]^{+}$, found 459.1428

1-(2-bromophenyl)-2-(2,4,6-trimethylbenzyl)-1,2,3,4-tetrahydropyrazino[1,2-a]indole $\mathbf{2 d}$

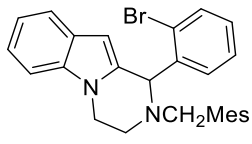

Compound $\mathbf{2 d}$ was synthesized following the General procedure 1 using tryptamine 5 a (44 mg, $0.15 \mathrm{mmol}, 1.0$ equiv.), 2-bromobenzaldehyde $(55 \mathrm{mg}, 0.30 \mathrm{mmol}, 2.0$ equiv.), Cat b (5.3 mg, $0.0075 \mathrm{mmol})$, in DCM (1.5 mL). The desired product $\mathbf{2 d}$ was obtained after column chromatography on silica gel (gradient from 0 to $50 \%$ Heptane:EtOAc) as a green amorphous solid ( $37 \mathrm{mg}, 0.083 \mathrm{mmol}, 55 \%$ ). IR (neat) $\boldsymbol{v}_{\max }=2922$, 2853, 1739, 1451, 1375, 1323, 1218, 1117, 1024cm-11H NMR $\left(\mathrm{CDCl}_{3}, 500 \mathrm{MHz}\right) \delta=7.54(\mathrm{~d}, J=7.9 \mathrm{~Hz}, 1 \mathrm{H}), 7.45(\mathrm{dd}, J=7.9,1.5$ $\mathrm{Hz}, 1 \mathrm{H}), 7.37(\mathrm{~d}, J=7.9 \mathrm{~Hz}, 1 \mathrm{H}), 7.21-7.14(\mathrm{~m}, 2 \mathrm{H}), 7.10(\mathrm{td}, J=5.0$, $1.5 \mathrm{~Hz}, 1 \mathrm{H}), 7.06(\mathrm{t}, J=5.0 \mathrm{~Hz}, 1 \mathrm{H}), 6.97(\mathrm{t}, J=4.9 \mathrm{~Hz}, 1 \mathrm{H}), 6.74(\mathrm{~s}$, $2 \mathrm{H}), 5.68(\mathrm{~s}, 1 \mathrm{H}), 5.25(\mathrm{~s}, 1 \mathrm{H}), 4.04(\mathrm{dt}, J=11.2,2.7 \mathrm{~Hz}, 1 \mathrm{H}), 3.87$ (dt, $J=11.2,4.2 \mathrm{~Hz}, 1 \mathrm{H}), 3.53(\mathrm{~d}, J=12.5 \mathrm{~Hz}, 1 \mathrm{H}), 3.42$ (d, $J=12.5$
$\mathrm{Hz}, 1 \mathrm{H}), 3.07(\mathrm{dq}, J=11.7,2.0 \mathrm{~Hz}, 1 \mathrm{H}), 2.74(\mathrm{td}, J=11.6,3.6 \mathrm{~Hz}$, 1H), $2.18(\mathrm{~s}, 3 \mathrm{H}), 2.16(6 \mathrm{H}) .{ }^{13} \mathrm{C}\left\{{ }^{1} \mathrm{H}\right\}$ NMR $\left(\mathrm{CDCl}_{3}, 75 \mathrm{MHz}\right) \delta=141.1$ $\left(\mathrm{C}_{\mathrm{q}}\right), 138.7\left(\mathrm{C}_{\mathrm{q}}\right), 138.4\left(\mathrm{C}_{\mathrm{q}}\right), 136.9\left(\mathrm{C}_{\mathrm{q}}\right), 135.7\left(\mathrm{C}_{\mathrm{q}}\right), 132.7(\mathrm{CH}), 132.6$ $(\mathrm{CH}), 131.2\left(\mathrm{C}_{\mathrm{q}}\right), 129.5(\mathrm{CH}), 129.3(\mathrm{CH}), 128.4\left(\mathrm{C}_{\mathrm{q}}\right), 127.6(\mathrm{CH})$, $125.4\left(\mathrm{C}_{\mathrm{q}}\right), 121.0(\mathrm{CH}), 120.4(\mathrm{CH}), 120.0(\mathrm{CH}), 108.8(\mathrm{CH}), 100.2$ $\left(\mathrm{C}_{\mathrm{q}}\right), 99.0(\mathrm{CH}), 66.3(\mathrm{CH}), 52.3\left(\mathrm{CH}_{2}\right), 47.5\left(\mathrm{CH}_{2}\right), 42.5\left(\mathrm{CH}_{2}\right), 21.1$ $\left(\mathrm{CH}_{3}\right), 20.8\left(\mathrm{CH}_{3}\right)$. HRMS (ESI): $\mathrm{m} / z$ : calc for $\mathrm{C}_{27} \mathrm{H}_{28} \mathrm{~N}_{2} \mathrm{Br}^{+} 459.1436$ $[\mathrm{M}+\mathrm{H}]^{+}$, found 459.1430

1-(4-(trifluoromethyl)phenyl)-2-(2,4,6-trimethylbenzyl)-1,2,3,4tetrahydropyrazino[1,2-a]indole $\mathbf{2 e}$

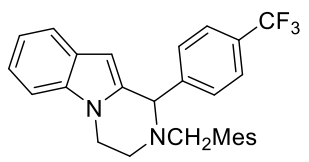

Compound $2 \mathrm{e}$ was synthesized following the General procedure 1 using tryptamine 5 a $(44 \mathrm{mg}, 0.15 \mathrm{mmol}, 1.0$ equiv.), 4-trifluorobenzaldehyde (52 $\mathrm{mg}, 0.30 \mathrm{mmol}, 2.0$ equiv.), Cat $\mathbf{b}$ ( 5.3 $\mathrm{mg}, 0.0075 \mathrm{mmol})$, in DCM $(1.5 \mathrm{~mL})$. The desired product $2 \mathrm{e}$ was obtained after column chromatography on silica gel (gradient from 0 to $50 \%$ Heptane:EtOAc) as a green amorphous solid (29 $\mathrm{mg}, 0.065 \mathrm{mmol}, 43 \%$ ). IR (neat) $\boldsymbol{v}_{\max }=2924,1739,1452,1366$, 1322, 1217, 1164, 1126, 1067, $1018 \mathrm{~cm}^{-1} .{ }^{1} \mathbf{H}$ NMR (CDCl, 500 MHz) $\delta=7.52(\mathrm{~d}, J=8.4 \mathrm{~Hz}, 2 \mathrm{H}), 7.46(\mathrm{~d}, J=7.9 \mathrm{~Hz}, 2 \mathrm{H}), 7.40$ (d, J $=7.9 \mathrm{~Hz}, 1 \mathrm{H}), 7.21(\mathrm{~d}, J=8.1 \mathrm{~Hz}, 1 \mathrm{H}), 7.10(\mathrm{t}, J=7.5 \mathrm{~Hz}, 1 \mathrm{H}), 7.01$ $(\mathrm{t}, J=7.5 \mathrm{~Hz}, 1 \mathrm{H}), 6.76(\mathrm{~s}, 2 \mathrm{H}), 5.70(\mathrm{~s}, 1 \mathrm{H}), 4.69(\mathrm{~s}, 1 \mathrm{H}), 4.07(\mathrm{dt}, J$ $=11.6,4.1 \mathrm{~Hz}, 1 \mathrm{H}), 3.92-3.85(\mathrm{~m}, 1 \mathrm{H}), 3.57(\mathrm{~d}, J=12.4 \mathrm{~Hz}, 1 \mathrm{H}), 3.33$ (d, $J=12.4 \mathrm{~Hz}, 1 \mathrm{H}$ ), 3.11 (dt, $J=12.2,4.2 \mathrm{~Hz}, 1 \mathrm{H}), 2.74$ (td, $J=10.8$, $3.8 \mathrm{~Hz}, 1 \mathrm{H}), 2.18(\mathrm{~s}, 3 \mathrm{H}), 2.17(\mathrm{~s}, 6 \mathrm{H}) .{ }^{13} \mathrm{C}\left\{{ }^{1} \mathrm{H}\right\} \mathrm{NMR}\left(\mathrm{CDCl}_{3}, 75 \mathrm{MHz}\right)$ $\delta=146.0\left(C_{q}\right), 138.5\left(C_{q}\right), 137.6\left(C_{q}\right), 137.1\left(C_{q}\right), 136.1\left(C_{q}\right), 131.1$ $\left(\mathrm{C}_{\mathrm{q}}\right), 130.0(\mathrm{CH}), 129.4(\mathrm{CH}), 128.3\left(\mathrm{C}_{\mathrm{q}}\right), 127.9\left(\mathrm{q}, \mathrm{C}-\mathrm{F},{ }^{1} \mathrm{~J}_{\mathrm{C}-\mathrm{F}}=267.1\right.$ $\left.\mathrm{Hz}, \mathrm{CF}_{3}\right), 125.3\left(\mathrm{q}, \mathrm{C}-\mathrm{F},{ }^{3} \mathrm{~J}_{\mathrm{C}-\mathrm{F}}=3.3 \mathrm{~Hz}, \mathrm{CH}\right), 121.3(\mathrm{CH}), 120.5(\mathrm{CH})$, $120.2(\mathrm{CH}), 109.0(\mathrm{CH}), 100.2\left(\mathrm{C}_{\mathrm{q}}\right), 100.0(\mathrm{CH}), 66.4(\mathrm{CH}), 52.4$ $\left(\mathrm{CH}_{2}\right), 46.7\left(\mathrm{CH}_{2}\right), 41.6\left(\mathrm{CH}_{2}\right), 21.1\left(\mathrm{CH}_{3}\right), 20.7\left(\mathrm{CH}_{3}\right) .{ }^{19} \mathrm{~F}$ NMR ( $\left.\mathrm{CDCl}_{3}, 282 \mathrm{MHz}\right) \delta=-62.4$. HRMS (ESI): $\mathrm{m} / \mathrm{z}$ : calc for $\mathrm{C}_{28} \mathrm{H}_{28} \mathrm{~N}_{2} \mathrm{~F}_{3}{ }^{+}$ $449.2205[\mathrm{M}+\mathrm{H}]^{+}$, found 449.2195

1-(3-(trifluoromethyl)phenyl)-2-(2,4,6-trimethylbenzyl)-1,2,3,4tetrahydropyrazino $[1,2-a]$ indole $\mathbf{2 f}$

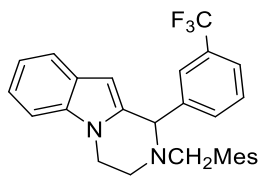

Compound $\mathbf{2 f}$ was synthesized following the General procedure 1 using tryptamine 5 a (44 mg, 0.15 mmol, 1.0 equiv.), 3 trifluorobenzaldehyde $(52 \mathrm{mg}, 0.30 \mathrm{mmol}$, 2.0 equiv.), Cat b ( $5.3 \mathrm{mg}, 0.0075 \mathrm{mmol})$, in

DCM $(1.5 \mathrm{~mL})$. The desired product $\mathbf{2} \mathrm{f}$ was obtained after column chromatography on silica gel (gradient from 0 to $50 \%$ Heptane:EtOAc) as a green amorphous solid ( $46 \mathrm{mg}, 0.10 \mathrm{mmol}, 67 \%$ ). IR (neat) $\boldsymbol{v}_{\max }=2921,1739,1451,1320,1163,1127,1072 \mathrm{~cm}^{-1} .{ }^{1} \mathbf{H}$ NMR $\left(\mathrm{CDCl}_{3}, 500 \mathrm{MHz}\right) \delta=7.76(\mathrm{~s}, 1 \mathrm{H}), 7.64(\mathrm{~d}, J=7.6 \mathrm{~Hz}, 1 \mathrm{H})$, $7.62(\mathrm{~d}, J=7.8 \mathrm{~Hz}, 1 \mathrm{H}), 7.50(\mathrm{~d}, J=8.4 \mathrm{~Hz}, 1 \mathrm{H}), 7.47(\mathrm{~d}, J=7.8 \mathrm{~Hz}$, $1 \mathrm{H}), 7.31(\mathrm{~d}, J=8.4 \mathrm{~Hz}, 1 \mathrm{H}), 7.20(\mathrm{t}, J=7.3 \mathrm{~Hz}, 1 \mathrm{H}), 7.11(\mathrm{t}, J=7.4$ $\mathrm{Hz}, 1 \mathrm{H}), 6.86(\mathrm{~s}, 2 \mathrm{H}), 5.78(\mathrm{~s}, 1 \mathrm{H}), 4.73(\mathrm{~s}, 1 \mathrm{H}), 4.16(\mathrm{dt}, J=10.6$, $3.7 \mathrm{~Hz}, 1 \mathrm{H}), 3.97(\mathrm{td}, J=10.7,4.3 \mathrm{~Hz}, 1 \mathrm{H}), 3.63(\mathrm{~d}, J=12.5 \mathrm{~Hz}, 1 \mathrm{H})$, $3.40(\mathrm{~d}, J=12.5 \mathrm{~Hz}, 1 \mathrm{H}), 3.20(\mathrm{dt}, J=12.3,4.1 \mathrm{~Hz}, 1 \mathrm{H}), 2.82(\mathrm{td}, J=$ $11,3.8 \mathrm{~Hz}, 1 \mathrm{H}), 2.28(\mathrm{~s}, 3 \mathrm{H}), 2.27(\mathrm{~s}, 6 \mathrm{H}) .{ }^{13} \mathrm{C}\left\{{ }^{1} \mathrm{H}\right\}$ NMR $\left(\mathrm{CDCl}_{3}, 75\right.$ $\mathrm{MHz}) \delta=143.0\left(\mathrm{C}_{\mathrm{q}}\right), 138.5\left(\mathrm{C}_{\mathrm{q}}\right), 137.9\left(\mathrm{C}_{\mathrm{q}}\right), 137.1\left(\mathrm{C}_{\mathrm{q}}\right), 136.1\left(\mathrm{C}_{\mathrm{q}}\right)$, $133.1(\mathrm{CH}), 131.0\left(\mathrm{C}_{\mathrm{q}}\right), 129.4(\mathrm{CH}), 128.7(\mathrm{CH}), 128.3\left(\mathrm{C}_{\mathrm{q}}\right), 128.0(\mathrm{q}$, $\left.\mathrm{C}-\mathrm{F},{ }^{1} J_{\mathrm{C}-\mathrm{F}}=274.4 \mathrm{~Hz}, \mathrm{CF}_{3}\right), 126.5\left(\mathrm{q}, \mathrm{C}-\mathrm{F},{ }^{3} \mathrm{~J}_{\mathrm{C}-\mathrm{F}}=3.5 \mathrm{~Hz}, \mathrm{CH}\right), 125.0$ (q, $\left.\mathrm{C}-\mathrm{F},{ }^{3} J_{\mathrm{C}-\mathrm{F}}=3.5 \mathrm{~Hz}, \mathrm{CH}\right), 121.3(\mathrm{CH}), 120.5(\mathrm{CH}), 120.2(\mathrm{CH}), 109.0$ (CH), $100.2\left(\mathrm{C}_{q}\right), 100.0(\mathrm{CH}), 66.9(\mathrm{CH}), 52.5\left(\mathrm{CH}_{2}\right), 47.0\left(\mathrm{CH}_{2}\right), 41.8$ $\left(\mathrm{CH}_{2}\right), 21.1\left(\mathrm{CH}_{3}\right), 20.6\left(\mathrm{CH}_{3}\right)$. HRMS (ESI): $\mathrm{m} / \mathrm{z}$ : calc for $\mathrm{C}_{28} \mathrm{H}_{28} \mathrm{~N}_{2} \mathrm{~F}_{3}{ }^{+}$ $449.2205[\mathrm{M}+\mathrm{H}]^{+}$, found 449.2217 . 
1-(3-methoxyphenyl)-2-(2,4,6-trimethylbenzyl)-1,2,3,4-tetrahydropyrazino[1,2-a]indole $\mathbf{2 g}$

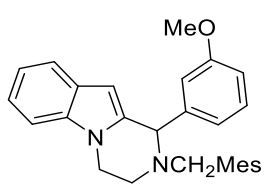

Compound $\mathbf{2 g}$ was synthesized following the General procedure 1 using tryptamine $5 \mathrm{a}(44 \mathrm{mg}, 0.15 \mathrm{mmol}, 1.0$ equiv.), 3-methoxybenzaldehyde (41 $\mathrm{mg}$, $0.30 \mathrm{mmol}, 2.0$ equiv. $)$, Cat $\mathbf{b}(5.3 \mathrm{mg}$ $0.0075 \mathrm{mmol})$, in DCM (1.5 mL). The desired product $\mathbf{2 g}$ was obtained after column chromatography on silica gel (gradient from 0 to $50 \%$ Heptane:EtOAc) as a green amorphous solid (36 mg, $0.088 \mathrm{mmol}, 59 \%$ ). IR (neat) $\boldsymbol{v}_{\max }=2952$, $1739,1599,1486,1452,1375,1321,1265,1148,1044 \mathrm{~cm}^{-1} .{ }^{1} \mathbf{H}$ NMR $\left(\mathrm{CDCl}_{3}, 500 \mathrm{MHz}\right) \delta=7.49(\mathrm{~d}, J=7.8 \mathrm{~Hz}, 1 \mathrm{H}), 7.30-7.25(\mathrm{~m}$, $2 \mathrm{H}), 7.17(\mathrm{td}, J=7.6,0.9 \mathrm{~Hz}, 1 \mathrm{H}), 7.08(\mathrm{td}, J=7.5,0.8 \mathrm{~Hz}, 1 \mathrm{H}), 7.05$ (d, $J=7.5 \mathrm{~Hz}, 1 \mathrm{H}$ ), 7.03-7.01 (m, $1 \mathrm{H}), 6.89$ (ddd, $J=8.2,2.6,0.5 \mathrm{~Hz}$, $1 \mathrm{H}), 6.84(\mathrm{~s}, 2 \mathrm{H}), 5.83(\mathrm{~s}, 1 \mathrm{H}), 4.64(\mathrm{~s}, 1 \mathrm{H}), 4.13(\mathrm{dt}, J=11.4,3.8 \mathrm{~Hz}$, $1 \mathrm{H}), 3.94$ (ddd, $J=4.5,10.2,11.2 \mathrm{~Hz}, 1 \mathrm{H}), 3.78(\mathrm{~s}, 3 \mathrm{H}), 3.71(\mathrm{~d}, J=$ $12.5 \mathrm{~Hz}, 1 \mathrm{H}), 3.36$ (d, $J=12.5 \mathrm{~Hz}, 1 \mathrm{H}), 3.18(\mathrm{dt}, J=12.1,4.0 \mathrm{~Hz}, 1 \mathrm{H})$, $2.78(\mathrm{~m}, 1 \mathrm{H}), 2.30(\mathrm{~s}, 6 \mathrm{H}), 2.28(\mathrm{~s}, 3 \mathrm{H}) .{ }^{13} \mathrm{C}\left\{{ }^{1} \mathrm{H}\right\}$ NMR $\left(\mathrm{CDCl}_{3}, 75\right.$ MHz) $\delta=159.7\left(C_{q}\right), 143.2\left(C_{q}\right), 138.8\left(C_{q}\right), 138.6\left(C_{q}\right), 136.8\left(C_{q}\right)$, $136.0\left(\mathrm{C}_{\mathrm{q}}\right), 131.5(\mathrm{Cq}), 129.3(\mathrm{CH}), 129.1(\mathrm{CH}), 128.4\left(\mathrm{C}_{\mathrm{q}}\right), 122.3$ $(\mathrm{CH}), 120.9(\mathrm{CH}), 120.4(\mathrm{CH}), 119.9(\mathrm{CH}), 114.9(\mathrm{CH}), 114,0(\mathrm{CH})$, $108.9(\mathrm{CH}), 100.2\left(\mathrm{C}_{\mathrm{q}}\right), 99.7(\mathrm{CH}), 67.5(\mathrm{CH}), 55.4\left(\mathrm{CH}_{2}\right), 47.0\left(\mathrm{CH}_{2}\right)$, $42.0\left(\mathrm{CH}_{2}\right), 21.1\left(\mathrm{CH}_{3}\right), 20.7\left(\mathrm{CH}_{3}\right) .{ }^{19} \mathrm{~F} \mathrm{NMR}\left(\mathrm{CDCl}_{3}, 282 \mathrm{MHz}\right) \delta=-$ 62.6 HRMS (ESI): $m / z$ : calc for $\mathrm{C}_{28} \mathrm{H}_{31} \mathrm{~N}_{2} \mathrm{O}^{+} 411.2436[\mathrm{M}+\mathrm{H}]^{+}$, found 411.2436

1-(quinolin-4-yl)-2-(2,4,6-trimethylbenzyl)-1,2,3,4-tetrahydropyrazino[1,2-a]indole $2 \mathrm{~h}$

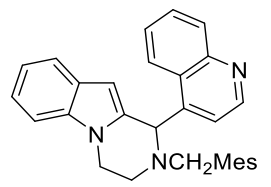

Compound $\mathbf{2 h}$ was synthesized following the General procedure 1 using tryptamine $5 a$ ( $44 \mathrm{mg}, 0.15 \mathrm{mmol}, 1.0$ equiv.), 4-quinolinaldehyde $(47 \mathrm{mg}, 0.30 \mathrm{mmol}, 2.0$ equiv.), Cat b (5.3 $\mathrm{mg}, 0.0075 \mathrm{mmol})$, in DCM $(1.5 \mathrm{~mL})$. The desired product $\mathbf{2} \mathbf{h}$ was obtained after column chromatography on silica gel (gradient from 0 to $100 \% \mathrm{Hep}$ tane:EtOAc) as a green amorphous solid (56 mg, $0.13 \mathrm{mmol}, 87 \%$ ). IR (neat) $\mathbf{v}_{\max }=2920,1739,1591,1452,1357,1218,1010 \mathrm{~cm}^{-1} .{ }^{1} \mathbf{H}$ NMR $\left(\mathrm{CDCl}_{3}, 500 \mathrm{MHz}\right) \delta=8.76(\mathrm{~d}, J=4.5 \mathrm{~Hz}, 1 \mathrm{hH}), 8.05$ (d, $J=8.3$ $\mathrm{Hz}, 1 \mathrm{H}), 7.76$ (d, J=8.5 Hz, 1H), 7.57 (td, $J=7.6,0.9 \mathrm{~Hz}, 1 \mathrm{H}), 7.39$ $(\mathrm{d}, J=7.8 \mathrm{~Hz}, 1 \mathrm{H}), 7.30(\mathrm{~d}, J=7.9 \mathrm{~Hz}, 1 \mathrm{H}), 7.23(\mathrm{~d}, J=7.5 \mathrm{~Hz}, 1 \mathrm{H})$, $7.22(\mathrm{~d}, J=5.2 \mathrm{~Hz}, 1 \mathrm{H}), 7.15(\mathrm{t}, J=7.6 \mathrm{~Hz}, 1 \mathrm{H}), 7.03(\mathrm{t}, J=7.5 \mathrm{~Hz}$, $1 \mathrm{H}), 6.79(\mathrm{~s}, 2 \mathrm{H}), 5.81(\mathrm{~s}, 1 \mathrm{H}), 5.34(\mathrm{~s}, 1 \mathrm{H}), 4.18(\mathrm{dt}, J=11.5,5.2 \mathrm{~Hz}$, $1 \mathrm{H}), 4.05(\mathrm{tt}, J=7.8,4.0 \mathrm{~Hz}, 1 \mathrm{H}), 3.66(\mathrm{~d}, J=12.7 \mathrm{~Hz}, 1 \mathrm{H}), 3.44(\mathrm{~d}$, $J=12.7 \mathrm{~Hz}, 1 \mathrm{H}), 3.26(\mathrm{dt}, J=12.7,5.0 \mathrm{~Hz}, 1 \mathrm{H}), 2.89-2.82(\mathrm{~m}, 1 \mathrm{H})$, $2.22(\mathrm{~s}, 3 \mathrm{H}), 2.11(\mathrm{~s}, 6 \mathrm{H}) .{ }^{13} \mathrm{C}\left\{{ }^{1} \mathrm{H}\right\}$ NMR $\left(\mathrm{CDCl}_{3}, 75 \mathrm{MHz}\right) \delta=150.0$ $(\mathrm{CH}), 149.1\left(\mathrm{C}_{\mathrm{q}}\right), 147.2\left(\mathrm{C}_{\mathrm{q}}\right), 138.7\left(\mathrm{C}_{\mathrm{q}}\right), 137.3\left(\mathrm{C}_{\mathrm{q}}\right), 136.2\left(\mathrm{C}_{\mathrm{q}}\right), 136.0$ $(\mathrm{Cq}), 130.7\left(\mathrm{C}_{\mathrm{q}}\right), 130.1(\mathrm{CH}), 129.4(\mathrm{CH}), 129.3(\mathrm{CH}), 128.3\left(\mathrm{C}_{\mathrm{q}}\right)$, $127.2\left(\mathrm{C}_{\mathrm{q}}\right), 126.2(\mathrm{CH}), 125.5(\mathrm{CH}), 122.7(\mathrm{CH}), 121.3(\mathrm{CH}), 120.6$ (CH), $120.3(\mathrm{CH}), 109.0(\mathrm{CH}), 101.2\left(\mathrm{C}_{\mathrm{q}}\right), 100.0(\mathrm{CH}), 62.7(\mathrm{CH}), 52.5$ $\left(\mathrm{CH}_{2}\right), 46.7\left(\mathrm{CH}_{2}\right), 40.7\left(\mathrm{CH}_{2}\right), 21.1\left(\mathrm{CH}_{3}\right), 20.7\left(\mathrm{CH}_{3}\right)$. HRMS (ESI): $\mathrm{m} / \mathrm{z}$ : calc for $\mathrm{C}_{30} \mathrm{H}_{30} \mathrm{~N}_{3}{ }^{+} 432.2440[\mathrm{M}+\mathrm{H}]^{+}$, found 432.2451 .

1-(isoquinolin-6-yl)-2-(2,4,6-trimethylbenzyl)-1,2,3,4-tetrahydropyrazino[1,2-a]indole $\mathbf{2} \mathbf{i}$

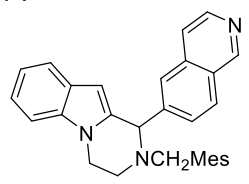

Compound $\mathbf{2} \mathbf{i}$ was synthesized following the General procedure 1 using tryptamine $\mathbf{5 a}$ (44 mg, $0.15 \mathrm{mmol}, 1.0$ equiv.), 6-quinolinaldehyde ( $47 \mathrm{mg}, 0.30 \mathrm{mmol}, 2.0$ equiv.), Cat b $(5.3 \mathrm{mg}, 0.0075 \mathrm{mmol})$, in DCM (1.5 $\mathrm{mL})$. The desired product $\mathbf{2} \mathbf{i}$ was obtained after column chromatography on silica gel (gradient from 0 to $100 \%$ Heptane:EtOAc) as a green amorphous solid (60 mg, $0.14 \mathrm{mmol}, 93 \%$ ). IR (neat) $\mathbf{v}_{\max }=2919,1738,1450$,
1357, 1312, 1265, 1116, $1010 \mathrm{~cm}^{-1} .{ }^{1} \mathrm{H} \mathrm{NMR}\left(\mathrm{CDCl}_{3}, 500 \mathrm{MHz}\right) \delta=$ $8.93(\mathrm{dd}, J=4.1,1.5 \mathrm{~Hz}, 1 \mathrm{H}$ ), $8.14(\mathrm{~d}, J=7.8 \mathrm{~Hz}, 1 \mathrm{H}$ ), 8.09 (d, $J=8.8$ $\mathrm{Hz}, 1 \mathrm{H}), 7.86(\mathrm{~d}, J=1.3 \mathrm{~Hz}, 1 \mathrm{H}), 7.80(\mathrm{dd}, J=8.7,1.9 \mathrm{~Hz}, 1 \mathrm{H}), 7.48$ (d, $J=7.9 \mathrm{~Hz}, 1 \mathrm{H}), 7.41(\mathrm{dd}, J=8.3,4.3 \mathrm{~Hz}, 1 \mathrm{H}), 7.31(\mathrm{~d}, J=8.0 \mathrm{~Hz}$, $1 \mathrm{H}), 7.19(\mathrm{t}, J=7.3 \mathrm{~Hz}, 1 \mathrm{H}), 7.10(\mathrm{t}, J=7.3 \mathrm{~Hz}, 1 \mathrm{H}), 6.84(\mathrm{~s}, 2 \mathrm{H})$, $5.80(\mathrm{~s}, 1 \mathrm{H}), 4.87(\mathrm{~s}, 1 \mathrm{H}), 4.17(\mathrm{dt}, J=11.5,3.9 \mathrm{~Hz}, 1 \mathrm{H}), 3.99(\mathrm{td}, J$ $=11.7,4.5 \mathrm{~Hz}, 1 \mathrm{H}), 3.71(\mathrm{~d}, J=12.6 \mathrm{~Hz}, 1 \mathrm{H}), 3.45(\mathrm{~d}, J=12.6 \mathrm{~Hz}$, $1 \mathrm{H}), 3.22(\mathrm{dt}, J=12.1,4.1 \mathrm{~Hz}, 1 \mathrm{H}), 2.85(\mathrm{td}, J=11.0,4.0 \mathrm{~Hz}, 1 \mathrm{H})$, $2.28(\mathrm{~s}, 6 \mathrm{H}), 2.27(\mathrm{~s}, 3 \mathrm{H}) .{ }^{13} \mathrm{C}\left\{{ }^{1} \mathrm{H}\right\}$ NMR $\left(\mathrm{CDCl}_{3}, 75 \mathrm{MHz}\right) \delta=150.6$ $(\mathrm{CH}), 148.5\left(\mathrm{C}_{\mathrm{q}}\right), 140.1\left(\mathrm{C}_{\mathrm{q}}\right), 138.5\left(\mathrm{C}_{\mathrm{q}}\right), 138.0\left(\mathrm{C}_{\mathrm{q}}\right), 136.9\left(\mathrm{C}_{\mathrm{q}}\right), 136.1$ $(\mathrm{CH}), 131.2(\mathrm{CH}), 131.1\left(\mathrm{C}_{\mathrm{q}}\right), 129.6(\mathrm{CH}), 129.3(\mathrm{CH}), 128.3(\mathrm{CH})$, $127.9\left(\mathrm{C}_{\mathrm{q}}\right), 121.4(\mathrm{CH}), 121.1(\mathrm{CH}), 120.5(\mathrm{CH}), 120.0(\mathrm{CH}), 108.9$ $(\mathrm{CH}), 100.1\left(\mathrm{C}_{\mathrm{q}}\right), 100.0(\mathrm{CH}), 66.9(\mathrm{CH}), 52.5\left(\mathrm{CH}_{2}\right), 46.8\left(\mathrm{CH}_{2}\right), 41.8$ $\left(\mathrm{CH}_{2}\right), 21.1\left(\mathrm{CH}_{3}\right), 20.7\left(\mathrm{CH}_{3}\right) \cdot$ HRMS (ESI): $\mathrm{m} / \mathrm{z}$ : calc for $\mathrm{C}_{30} \mathrm{H}_{30} \mathrm{~N}_{3}{ }^{+}$ $432.2440[\mathrm{M}+\mathrm{H}]^{+}$, found 432.2427

\section{C2-Iso Pictet-Spengler reaction}

Synthesis of 2-(1H-indol-2-yl)-N-(2,4,6-trimethylbenzyl)ethan-1amine 5b

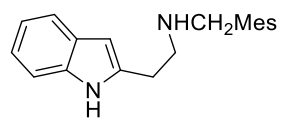

The synthesis of 2-(1H-indol-2-yl)ethan1-amine was performed following procedures from the literature. ${ }^{8 d, 23}$ To a solution of Indole-2-carboxylic acid (16.1 g, 1.00 equiv., $100 \mathrm{mmol})$ in THF $(75 \mathrm{~mL})$ was added $\mathrm{LiAlH}_{4}(7.9 \mathrm{~g}$, 2.07 equiv., $207 \mathrm{mmol}$ ) at $0 \cong \mathrm{C}$ in a $15 \mathrm{~min}$ period, the mixture was then allowed to warm to room temperature slowly and stirring at room temperature for about $3 \mathrm{~h}$. The reaction was quenched with $2 \mathrm{M} \mathrm{NaOH}$ under $0 \stackrel{\circ}{ } \mathrm{C}$ and extracted with EtOAc, dried over $\mathrm{MgSO}_{4}$ and evaporated under vacuum. The crude product was then purified by column chromatography to afford the corresponding $(1 \mathrm{H}-$ indol-2-yl)methanol in $84 \%$ yield $(12.4 \mathrm{~g}, 84 \mathrm{mmol})$. To a solution of (1H-indol-2-yl)methanol (12.4 g, 1 equiv., $84 \mathrm{mmol})$ in $\mathrm{MeCN}$ (360 mL) was added $\mathrm{MnO}_{2}$ (73 g, 10 equiv., $840 \mathrm{mmol}$ ) at room temperature and the mixture was stirred for $16 \mathrm{~h}$, then the mixture was filtrated with celite and concentrated under vacuum. The crude product was used directly without purification. To the solution of crude $1 \mathrm{H}$-indole-2-carbaldehyde ( $11.0 \mathrm{~g}, 1.00$ equiv., 76.1 $\mathrm{mmol}$ ) in $\mathrm{MeNO}_{2}(70 \mathrm{~mL})$ was added $\mathrm{AcONH}_{4}(2.3 \mathrm{~g}, 0.39 \mathrm{mmol}$, $29.7 \mathrm{mmol}$ ) at room temperature, the mixture was allowed heated to $100 \stackrel{\circ}{\circ}$ and following by TLC, 30 min later, the reaction was completed and concentrated under vacuum, the residue was then extracted with EtOAc and dried over $\mathrm{MgSO}_{4}$ and evaporated under vacuum, leading to 2-(2-nitrovinyl)- $1 H$-indole. It was then dissolved in THF ( $50 \mathrm{~mL})$ and a suspension of $\mathrm{LiAlH}_{4}(5.5 \mathrm{~g}, 1$ equiv., $29 \mathrm{mmol})$ in THF (50 mL) was added dropwise at 0 으, after completed, the resulting mixture was stirred at room temperature for $3.5 \mathrm{~h}$. The reaction solution was then quenched with $\mathrm{NH}_{4} \mathrm{Cl}$ and extracted with EtOAc, combine the organic layer and dry over $\mathrm{MgSO}_{4}$, concentrated under vacuum and purified by column chromatography to afford the corresponding 2-(1H-indol-2-yl)ethan-1amine (2.4 g, $15 \mathrm{mmol}, 52 \%) .{ }^{24}$

A mixture of 2 -( $1 \mathrm{H}$-indol-2-yl)ethan-1-amine $(481 \mathrm{mg}, 1.00$ equiv., $3.00 \mathrm{mmol}$ ) and mesitaldehyde (467 mg, 1.05 equiv., $3.15 \mathrm{mmol}$ ) in $\mathrm{MeOH}\left(5 \mathrm{~mL}\right.$ ) under $\mathrm{N}_{2}$ was stirred at room temperature for 16 hours, then $\mathrm{NaBH}_{4}$ was added under $0 \circ \mathrm{C}$, the mixture was allowed warm to room temperature and stirred at room temperature for another $1 \mathrm{~h}$ and water was added. After the phases were separated, the aqueous phase was extracted twice by ethyl acetate then the combined organic phases were dried over $\mathrm{MgSO}_{4}$ and evaporated under vacuum. and extraction with EtOAc, combine the organic layer and dry over $\mathrm{MgSO}_{4}$, concentrated under vacuum and purified by column chromatography to afford the corresponding $\quad 2-(1 H$-indol-2-yl)- $N-(2,4,6$-trimethylbenzyl)ethan-1- 
amine 5b (265 mg, $0.91 \mathrm{mmol}, 30 \%) .{ }^{1} \mathrm{H} \mathrm{NMR} \mathrm{(CDCl_{3 } , 5 0 0 ~ M H z ) ~} \delta$ $=9.71(\mathrm{bs}, 1 \mathrm{H}), 7.51(\mathrm{~d}, J=7.6 \mathrm{~Hz}, 1 \mathrm{H}), 7.24(\mathrm{~d}, J=7.9 \mathrm{~Hz}, 1 \mathrm{H}), 7.09$ $(\mathrm{t}, J=7.0 \mathrm{~Hz}, 1 \mathrm{H}), 7.04(\mathrm{t}, J=7.3 \mathrm{~Hz}, 1 \mathrm{H}), 6.89(\mathrm{~s}, 1 \mathrm{H}), 6.20(\mathrm{~s}, 1 \mathrm{H})$, $3.81(\mathrm{~s}, 2 \mathrm{H}), 3.08(\mathrm{t}, J=5.8 \mathrm{~Hz}, 2 \mathrm{H}), 2.93(\mathrm{t}, J=5.8 \mathrm{~Hz}, 2 \mathrm{H}), 2.39(\mathrm{~s}$, $6 \mathrm{H}), 2.28(\mathrm{~s}, 3 \mathrm{H}), 1.42(\mathrm{~m}, 1 \mathrm{H}) \cdot{ }^{13} \mathrm{C}\left\{{ }^{1} \mathrm{H}\right\} \mathrm{NMR}\left(\mathrm{CDCl}_{3}, 125 \mathrm{MHz}\right) \delta=$ $139.6\left(C_{q}\right), 137.1\left(C_{q}\right), 137.1\left(C_{q}\right), 136.0\left(C_{q}\right), 133.4\left(C_{q}\right), 129.4(C H)$, $128.6\left(\mathrm{C}_{\mathrm{q}}\right), 121.0(\mathrm{CH}), 119.9(\mathrm{CH}), 119.5(\mathrm{CH}), 110.7(\mathrm{CH}), 99.3$ $(\mathrm{CH}), 49.8\left(\mathrm{CH}_{2}\right), 47.9\left(\mathrm{CH}_{2}\right), 28.1\left(\mathrm{CH}_{2}\right), 21.1\left(\mathrm{CH}_{3}\right), 19.7\left(\mathrm{CH}_{3}\right)$. HRMS (ESI): $m / z$ : calc for $\mathrm{C}_{20} \mathrm{H}_{25} \mathrm{~N}_{2}{ }^{+}$293.2012, [M+H] $]^{+}$, found, 293.2009.

General procedure 2 for the synthesis of tetrahydro- $1 \mathrm{H}$ pyrido[4,3-b]indole 3: A mixture of 2-(1H-indol-2-yl)- $N-(2,4,6$-trimethylbenzyl)ethan-1-amine $5 \mathbf{b}$ ( 1 equiv), Cat b $(5 \mathrm{~mol} \%)$ and $3 \AA$ molecular sieves ( $150 \mathrm{mg}$ for $0.15 \mathrm{mmol}$ of $\mathbf{5 b}$, powdered) in dichloromethane ( $1.5 \mathrm{~mL}$ for $0.15 \mathrm{mmol}$ of $5 \mathrm{a}$ ) was stirred for $5 \mathrm{~min}$ at room temperature under an argon atmosphere. Subsequently, aldehyde ( 2.0 equiv) was added and the mixture stirred at $-20^{\circ} \mathrm{C}$ for $16 \mathrm{~h}$. Then it was filtered under celite and silica was added. After evaporation of the volatiles, the silica mixture was purified by chromatography under silica gel to give the desired product 3 . 1-(4-bromophenyl)-2-(2,4,6-trimethylbenzyl)-2,3,4,5-tetrahydro$1 H$-pyrido[4,3-b]indole $3 a$

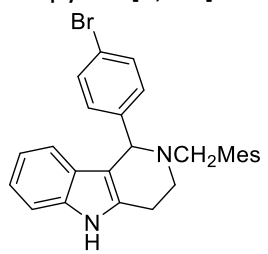

Compound 3a was prepared according to the general procedure $\mathbf{2}$ from $\mathbf{5 b}(0.05$ mmol, 1 equiv., $14.6 \mathrm{mg}$ ), 4-bromobenzaldehyde ( $0.1 \mathrm{mmol}, 2$ equiv., $18.5 \mathrm{mg}$ ), Cat b $(5 \mathrm{~mol} \%, 1.8 \mathrm{mg}), 3 \AA \circ \cdot \mathrm{MS}(50 \mathrm{mg})$ and anhydrous DCM under $\mathrm{N}_{2}$, yielding product 3a (12.7 $\mathrm{mg}, 0.028 \mathrm{mmol}, 56 \%)$ as a pale yellow solid. ${ }^{1} \mathbf{H}$ NMR $\left(\mathrm{CDCl}_{3}, \mathbf{3 0 0} \mathbf{~ M H z}\right) \delta=7.84$ (bs, $\left.1 \mathrm{H}\right), 7.37$ (d, $J$ $=8.3 \mathrm{~Hz}, 2 \mathrm{H}), 7.29(\mathrm{~d}, J=7.9 \mathrm{~Hz}, 1 \mathrm{H}), 7.17(\mathrm{~d}, J=8.5 \mathrm{~Hz}, 2 \mathrm{H}), 7.11-$ $7.05(\mathrm{~m}, 1 \mathrm{H}), 6.93-6.88(\mathrm{~m}, 1 \mathrm{H}), 6.83-6.79(\mathrm{~m}, 3 \mathrm{H}), 4.64(\mathrm{~s}, 1 \mathrm{H})$, $3.77(\mathrm{~d}, J=12.4 \mathrm{~Hz}, 1 \mathrm{H}), 3.48(\mathrm{~d}, J=12.4 \mathrm{~Hz}, 1 \mathrm{H}), 3.09-2.99(\mathrm{~m}$, $1 \mathrm{H}), 2.84-2.69(\mathrm{~m}, 3 \mathrm{H}), 2.27(\mathrm{~s}, 3 \mathrm{H}), 2.19(\mathrm{~s}, 6 \mathrm{H}) .{ }^{13} \mathrm{C}\left\{{ }^{1} \mathrm{H}\right\}$ NMR $\left(\mathrm{CDCl}_{3}, 75 \mathrm{MHz}\right) \delta=142.6\left(\mathrm{C}_{\mathrm{q}}\right), 138.6\left(\mathrm{C}_{\mathrm{q}}\right), 136.6\left(\mathrm{C}_{\mathrm{q}}\right), 136.1\left(\mathrm{C}_{\mathrm{q}}\right)$, $133.5\left(\mathrm{C}_{\mathrm{q}}\right), 132.5\left(\mathrm{C}_{\mathrm{q}}\right), 131.4(\mathrm{CH}), 131.1(\mathrm{CH}), 129.2(\mathrm{CH}), 126.9$ $\left(\mathrm{C}_{\mathrm{q}}\right), 121.4(\mathrm{CH}), 121.0\left(\mathrm{C}_{\mathrm{q}}\right), 119.7(\mathrm{CH}), 118.7(\mathrm{CH}), 111.0\left(\mathrm{C}_{\mathrm{q}}\right)$, $110.7(\mathrm{CH}), 62.6(\mathrm{CH}), 51.2\left(\mathrm{CH}_{2}\right), 45.8\left(\mathrm{CH}_{2}\right), 22.7\left(\mathrm{CH}_{2}\right), 21.1\left(\mathrm{CH}_{3}\right)$, $20.4\left(\mathrm{CH}_{3}\right)$. HRMS (ESI): $\mathrm{m} / \mathrm{z}$ : calc for $\mathrm{C}_{27} \mathrm{H}_{28} \mathrm{BrN}_{2}{ }^{+} 459.1430[\mathrm{M}+\mathrm{H}]^{+}$, found, 459.1437.

1-(quinolin-4-yl)-2-(2,4,6-trimethylbenzyl)-2,3,4,5-tetrahydro- $1 \mathrm{H}$ pyrido $[4,3-b]$ indole $\mathbf{3 b}$

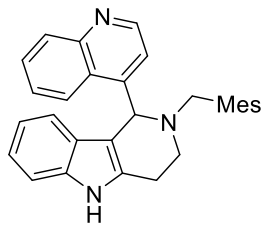

Compound $\mathbf{3 b}$ was prepared according to the general procedure $\mathbf{2}$ from $\mathbf{5 b}(0.05$ mmol, 1 equiv., $14.6 \mathrm{mg}$ ), quinoline-4carbaldehyde $(0.1 \mathrm{mmol}, 2$ equiv., 15.7 $\mathrm{mg})$, Cat b (5 mol \%, $1.8 \mathrm{mg}), 3 \AA \circ \cdot \mathrm{MS}(50$ $\mathrm{mg}$ ) and anhydrous DCM under $\mathrm{N}_{2}$, yielding product $\mathbf{3 b}(13.1 \mathrm{mg}, 0.03 \mathrm{mmol}, 61 \%)$ as a pale yellow solid. ${ }^{1} \mathrm{H}$ NMR $\left(\mathrm{CDCl}_{3}, 500 \mathrm{MHz}\right) \delta=8.68(\mathrm{~s}, 1 \mathrm{H}), 8.09(\mathrm{~d}, J=8.2 \mathrm{~Hz}, 1 \mathrm{H})$, $8.04(\mathrm{~s}, 1 \mathrm{H}), 7.62(\mathrm{t}, J=7.6 \mathrm{~Hz}, 1 \mathrm{H}), 7.55$ (bs, 1H), 7.36 (d, $J=8.1$ $\mathrm{Hz}, 1 \mathrm{H}), 7.21(\mathrm{t}, J=7.6 \mathrm{~Hz}, 1 \mathrm{H}), 7.11(\mathrm{t}, J=7.5 \mathrm{~Hz}, 1 \mathrm{H}$ ), 7.03 (bs, $1 \mathrm{H}), 6.91(\mathrm{~s}, 2 \mathrm{H}), 6.86(\mathrm{t}, J=7.5 \mathrm{~Hz}, 1 \mathrm{H}), 6.78(\mathrm{~d}, J=7.8 \mathrm{~Hz}, 1 \mathrm{H})$, $5.51(\mathrm{~s}, 1 \mathrm{H}), 3.99(\mathrm{~d}, J=12.4 \mathrm{~Hz}, 1 \mathrm{H}), 3.63(\mathrm{~d}, J=12.5 \mathrm{~Hz}, 1 \mathrm{H}), 3.19-$ $3.09(\mathrm{~m}, 2 \mathrm{H}), 2.99-2.90(\mathrm{~m}, 1 \mathrm{H}), 2.83-2.74(\mathrm{~m}, 1 \mathrm{H}), 2.34(\mathrm{~s}, 3 \mathrm{H})$, $2.14(\mathrm{~s}, 6 \mathrm{H}) .{ }^{13} \mathrm{C}\left\{{ }^{1} \mathrm{H}\right\} \mathrm{NMR}\left(\mathrm{CDCl}_{3}, 125 \mathrm{MHz}\right) \delta=150.0(\mathrm{CH}), 148.8$ $\left(C_{q}\right), 148.3\left(C_{q}\right), 139.1\left(C_{q}\right), 137.1\left(C_{q}\right), 136.1\left(C_{q}\right), 133.6\left(C_{q}\right), 131.9$ $\left(\mathrm{C}_{q}\right), 131.2\left(\mathrm{C}_{q}\right), 130.4(\mathrm{CH}), 129.8(\mathrm{CH}), 129.3(\mathrm{CH}), 128.9(\mathrm{CH})$, $128.0\left(\mathrm{C}_{\mathrm{q}}\right), 125.8(\mathrm{CH}), 125.1\left(\mathrm{C}_{\mathrm{q}}\right), 122.4(\mathrm{CH}), 121.7(\mathrm{CH}), 119.8$ $(\mathrm{CH}), 118.4(\mathrm{CH}), 110.8(\mathrm{CH}), 50.9\left(\mathrm{CH}_{2}\right), 46.8(\mathrm{CH}), 45.9\left(\mathrm{CH}_{2}\right), 21.2$ $\left(\mathrm{CH}_{2}\right), 20.5\left(\mathrm{CH}_{3}\right), 20.2\left(\mathrm{CH}_{3}\right)$. HRMS (ESI): $\mathrm{m} / \mathrm{z}$ : calc for $\mathrm{C}_{30} \mathrm{H}_{30} \mathrm{~N}_{3}{ }^{+}$ $432.2434[\mathrm{M}+\mathrm{H}]^{+}$, found, 432.2436.
1-(pyridin-4-yl)-2-(2,4,6-trimethylbenzyl)-2,3,4,5-tetrahydro-1Hpyrido[4,3-b]indole $3 c$

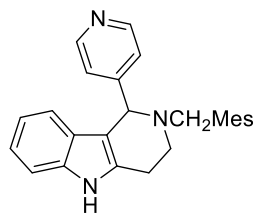

Compound $\mathbf{3 c}$ was prepared according to the general procedure 2 from $5 c(0.05$ mmol, 1 equiv., $14.6 \mathrm{mg}$ ), isonicotinaldehyde ( $0.1 \mathrm{mmol}, 2$ equiv., $10.7 \mathrm{mg})$, Cat b $(5$ $\mathrm{mol} \%, 1.8 \mathrm{mg}$ ), 3Å.MS (50 mg) and anhydrous DCM under $\mathrm{N}_{2}$, yielding product $3 \mathrm{C}$ (13.4 mg, $0.04 \mathrm{mmol}, 71 \%$ ) as a pale yellow solid. ${ }^{1} \mathbf{H}$ NMR $\left(\mathrm{CDCl}_{3}\right.$, $300 \mathrm{MHz}) \delta=8.47(\mathrm{~d}, J=3.2 \mathrm{~Hz}, 2 \mathrm{H}), 8.15(\mathrm{~s}, 1 \mathrm{H}), 7.34(\mathrm{~d}, J=8.1$ $\mathrm{Hz}, 1 \mathrm{H}), 7.23(\mathrm{~d}, J=5.5 \mathrm{~Hz}, 2 \mathrm{H}), 7.15-7.07(\mathrm{~m}, 1 \mathrm{H}), 6.98-6.87(\mathrm{~m}$, $2 \mathrm{H}), 6.85(\mathrm{~s}, 2 \mathrm{H}), 4.73(\mathrm{~s}, 1 \mathrm{H}), 3.82(\mathrm{~d}, J=12.4 \mathrm{~Hz}, 1 \mathrm{H}), 3.56(\mathrm{~d}, J=$ $12.5 \mathrm{~Hz}, 1 \mathrm{H}), 3.07-2.83(\mathrm{~m}, 3 \mathrm{H}), 2.77-2.66(\mathrm{~m}, 1 \mathrm{H}), 2.28(\mathrm{~s}, 3 \mathrm{H})$, $2.20(\mathrm{~s}, 6 \mathrm{H}) .{ }^{13} \mathrm{C}\left\{{ }^{1} \mathrm{H}\right\}$ NMR $\left(\mathrm{CDCl}_{3}, 75 \mathrm{MHz}\right) \delta=153.7\left(\mathrm{C}_{\mathrm{q}}\right), 148.9$ $(\mathrm{CH}), 138.5\left(\mathrm{C}_{\mathrm{q}}\right), 136.8\left(\mathrm{C}_{\mathrm{q}}\right), 136.0\left(\mathrm{C}_{\mathrm{q}}\right), 133.6\left(\mathrm{C}_{\mathrm{q}}\right), 132.1\left(\mathrm{C}_{\mathrm{q}}\right), 129.3$ $(\mathrm{CH}), 126.9\left(\mathrm{C}_{\mathrm{q}}\right), 124.8(\mathrm{CH}), 121.6(\mathrm{CH}), 119.9(\mathrm{CH}), 118.3(\mathrm{CH})$, $110.9(\mathrm{CH}), 109.2\left(\mathrm{C}_{\mathrm{q}}\right), 60.6(\mathrm{CH}), 51.0\left(\mathrm{CH}_{2}\right), 45.5\left(\mathrm{CH}_{2}\right), 21.7\left(\mathrm{CH}_{2}\right)$, $21.1\left(\mathrm{CH}_{3}\right), 20.2\left(\mathrm{CH}_{3}\right)$. HRMS (ESI): $\mathrm{m} / \mathrm{z}$ : calc for $\mathrm{C}_{26} \mathrm{H}_{28} \mathrm{~N}_{3}{ }^{+}$ 382.2278 $[\mathrm{M}+\mathrm{H}]^{+}$, found, 382.2272.

3-(2-(2,4,6-trimethylbenzyl)-2,3,4,5-tetrahydro-1H-pyrido[4,3b]indol-1-yl)benzaldehyde $\mathbf{3 d}$

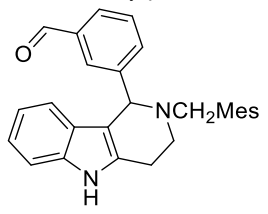

Compound 3d was prepared according to the general procedure 2 from $5 c(0.05$ $\mathrm{mmol}, 1$ equiv., $14.6 \mathrm{mg}$ ), isophthalaldehyde $(0.1 \mathrm{mmol}, 2$ equiv., $10.7 \mathrm{mg})$, Cat $\mathbf{b}$ (5 mol \%, $1.8 \mathrm{mg}$ ), $3 \AA \AA$ A $\mathrm{MS}$ (50 mg) and anhydrous DCM under $\mathrm{N}_{2}$, yielding product 3d (15.4 mg, $0.04 \mathrm{mmol}, 75 \%$ ) as a pale yellow solid. ${ }^{1} \mathbf{H}$ NMR $\left(\mathrm{CDCl}_{3}, 300 \mathrm{MHz}\right) \delta=9.95(\mathrm{~s}, 1 \mathrm{H}), 7.90$ (bs, $\left.1 \mathrm{H}\right), 7.84(\mathrm{t}, J=1.5 \mathrm{~Hz}$, $1 \mathrm{H}), 7.77\left(\mathrm{dt}, J_{1}=7.5 \mathrm{~Hz}, J_{2}=1.3 \mathrm{~Hz}, 1 \mathrm{H}\right), 7.58\left(\mathrm{dt}, J_{1}=7.7 \mathrm{~Hz}, J_{2}=\right.$ $1.3 \mathrm{~Hz}, 1 \mathrm{H}), 7.42(\mathrm{t}, J=7.5 \mathrm{~Hz}, 1 \mathrm{H}), 7.30\left(\mathrm{dt}, J_{1}=8.1 \mathrm{~Hz}, J_{2}=0.8 \mathrm{~Hz}\right.$, $1 \mathrm{H}), 7.11-7.04(\mathrm{~m}, 1 \mathrm{H}), 6.90-6.85(\mathrm{~m}, 1 \mathrm{H}), 6.83(\mathrm{~s}, 2 \mathrm{H}), 6.77(\mathrm{~d}, J=$ $7.9 \mathrm{~Hz}, 1 \mathrm{H}), 4.77(\mathrm{~s}, 1 \mathrm{H}), 3.76(\mathrm{~d}, J=12.4 \mathrm{~Hz}, 1 \mathrm{H}), 3.53(\mathrm{~d}, J=12.4$ $\mathrm{Hz}, 1 \mathrm{H}), 3.12-3.00(\mathrm{~m}, 1 \mathrm{H}), 2.85-2.73(\mathrm{~m}, 3 \mathrm{H}), 2.27(\mathrm{~s}, 3 \mathrm{H}), 2.18(\mathrm{~s}$, 6H). ${ }^{13} \mathrm{C}\left\{{ }^{1} \mathrm{H}\right\}$ NMR $\left(\mathrm{CDCl}_{3}, 75 \mathrm{MHz}\right) \delta=192.9(\mathrm{CH}), 144.9\left(\mathrm{C}_{\mathrm{q}}\right), 138.6$ $\left(C_{q}\right), 136.6\left(C_{q}\right), 136.3\left(C_{q}\right), 136.2\left(C_{q}\right), 136.0(C H), 133.6\left(C_{q}\right), 132.4$ $\left(\mathrm{C}_{\mathrm{q}}\right), 131.3(\mathrm{CH}), 129.2(\mathrm{CH}), 128.8(\mathrm{CH}), 128.6(\mathrm{CH}), 126.7\left(\mathrm{C}_{\mathrm{q}}\right)$, $121.4(\mathrm{CH}), 119.7(\mathrm{CH}), 118.5(\mathrm{CH}), 110.8(\mathrm{CH}), 63.0(\mathrm{CH}), 51.3$ $\left(\mathrm{CH}_{2}\right), 45.9\left(\mathrm{CH}_{2}\right), 22.9\left(\mathrm{CH}_{2}\right), 21.1\left(\mathrm{CH}_{3}\right), 20.4\left(\mathrm{CH}_{3}\right)$. HRMS (ESI): $\mathrm{m} / \mathrm{z}$ : calc for $\mathrm{C}_{28} \mathrm{H}_{29} \mathrm{~N}_{2} \mathrm{O}^{+} 409.2274[\mathrm{M}+\mathrm{H}]^{+}$, found, 409.2273 .

N-benzyl-1-(4-(2-(2,4,6-trimethylbenzyl)-2,3,4,5-tetrahydro-1H-

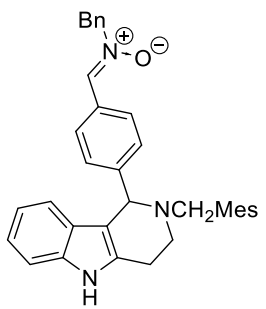
pyrido[4,3-b]indol-1-yl)phenyl)methanimine oxide $\mathbf{3 e}$ Compound $3 \mathrm{e}$ was prepared according to the general procedure 2 from $\mathbf{5 c}(0.05$ mmol, 1 equiv., $14.6 \mathrm{mg}),(Z)-N$-benzyl-1(4-formylphenyl)methanimine oxide $(0.1$ mmol, 2 equiv., $23.9 \mathrm{mg}$ ), Cat b $(5 \mathrm{~mol} \%$, $1.8 \mathrm{mg}), 3 \AA$ A $\cdot \mathrm{MS}(50 \mathrm{mg})$ and anhydrous DCM under $\mathrm{N}_{2}$, stirred at room temperature for 60 hours, yielding product $3 e(65 \%$ conversion, $12.1 \mathrm{mg}, 0.02 \mathrm{mmol}, 47 \%$ ) as a white solid.

Note: the background reaction performed under the same conditions in the absence of catalyst is $26 \%$ after $60 \mathrm{~h} .{ }^{1} \mathrm{H} \mathrm{NMR}\left(\mathrm{CDCl}_{3}\right.$, $500 \mathrm{MHz}) \delta=8.11(\mathrm{~d}, J=7.9 \mathrm{~Hz}, 2 \mathrm{H}), 7.88(\mathrm{~s}, 1 \mathrm{H}), 7.47(\mathrm{~d}, J=6.6$ $\mathrm{Hz}, 2 \mathrm{H}), 7.42-7.33(\mathrm{~m}, 6 \mathrm{H}), 7.25(\mathrm{~d}, J=7.9 \mathrm{~Hz}, 1 \mathrm{H}), 7.03(\mathrm{t}, J=7.6$ $\mathrm{Hz}, 1 \mathrm{H}), 6.82(\mathrm{t}, J=7.5 \mathrm{~Hz}, 1 \mathrm{H}), 6.80(\mathrm{~s}, 2 \mathrm{H}), 6.73(\mathrm{~d}, J=7.6 \mathrm{~Hz}, 1 \mathrm{H})$, $5.04(\mathrm{~s}, 2 \mathrm{H}), 4.67(\mathrm{~s}, 1 \mathrm{H}), 3.73(\mathrm{~d}, J=12.4 \mathrm{~Hz}, 1 \mathrm{H}), 3.47(\mathrm{~d}, J=12.5$ $\mathrm{Hz}, 1 \mathrm{H}), 3.06-2.98(\mathrm{~m}, 1 \mathrm{H}), 2.81-2.67(\mathrm{~m}, 3 \mathrm{H}), 2.25(\mathrm{~s}, 3 \mathrm{H}), 2.17(\mathrm{~s}$, $6 \mathrm{H}) .{ }^{13} \mathrm{C}\left\{{ }^{1} \mathrm{H}\right\} \mathrm{NMR}\left(\mathrm{CDCl}_{3}, 125 \mathrm{MHz}\right) \delta=146.4\left(\mathrm{C}_{\mathrm{q}}\right), 138.6\left(\mathrm{C}_{\mathrm{q}}\right), 136.5$ $\left(C_{q}\right), 136.1\left(C_{q}\right), 134.7(C H), 133.5\left(C_{q}\right), 133.4\left(C_{q}\right), 132.5\left(C_{q}\right), 129.9$ 
(CH), $129.5(\mathrm{CH}), 129.5\left(\mathrm{C}_{\mathrm{q}}\right) 129.2(\mathrm{CH}), 129.2(\mathrm{CH}), 129.1(\mathrm{CH})$, $128.5(\mathrm{CH}), 126.7\left(\mathrm{C}_{\mathrm{q}}\right), 121.2(\mathrm{CH}), 119.6(\mathrm{CH}), 118.7(\mathrm{CH}), 111.3$ $\left(\mathrm{C}_{\mathrm{q}}\right), 110.6(\mathrm{CH}), 71.3\left(\mathrm{CH}_{2}\right), 63.9(\mathrm{CH}), 51.5\left(\mathrm{CH}_{2}\right), 46.0\left(\mathrm{CH}_{2}\right), 23.2$ $\left(\mathrm{CH}_{2}\right), 21.1\left(\mathrm{CH}_{3}\right), 20.5\left(\mathrm{CH}_{3}\right)$. HRMS (ESI): $\mathrm{m} / \mathrm{z}$ : calc for $\mathrm{C}_{35} \mathrm{H}_{36} \mathrm{~N}_{3} \mathrm{O}^{+}$ $514.2853[\mathrm{M}+\mathrm{H}]^{+}$, found, 514.2847.

\section{C4-Iso Pictet-Spengler reaction}

\section{Synthesis of tryptamine $5 \mathrm{c}$.}

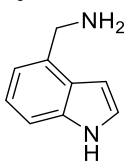

A mixture of $1 \mathrm{H}$-indole-4-carbonitrile (10.0 g, 70.3 mmol, 1.0 equiv.) in THF (100 mL) was added dropwise to a suspension of lithium aluminum hydride ( $5.70 \mathrm{~g}, 150 \mathrm{mmol}, 2.1$ equiv.) in $\mathrm{THF}$ at $0{ }^{\circ} \mathrm{C}$. The reaction was refluxed for 90 minutes then allowed to cool to rt. Then, aqueous saturated solution of Rochelle's salt was added and stirred until full destruction of lithium salts. The reaction was filtered over silica, concentrated under vacuum and triturated in methanol. After filtration over celite, the pure product S6 was obtained by filtrate's concentration under vacuum as a light brown solid $(9.10 \mathrm{~g}, 62.2 \mathrm{mmol}, 88 \%)$. The data correspond to those found in the literature. ${ }^{25}$

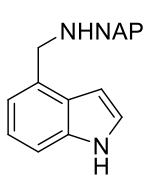

(1H-indol-4-yl)methanamine $(2.00 \mathrm{mg}, 13.6 \mathrm{mmol}$, 1.00 equiv.), and 2-naphthaldehyde ( $2.02 \mathrm{mg}, 13.0$ $\mathrm{mmol}, 0.95$ equiv.) were stirred in methanol (68 $\mathrm{mL}$ ) for 4 hours. Then, the reaction media was cooled to $0{ }^{\circ} \mathrm{C}$ before the addition of $\mathrm{NaBH}_{4}$ (565 $\mathrm{mg}, 15.0 \mathrm{mmol}, 1.1$ equiv. in one portion). The mixture was allowed to warm up to room temperature. After 1 hour stirring, the volatiles were removed and the crude was next diluted in ethyl acetate and water. After phases separation, the aqueous phase was extracted twice by ethyl acetate. The combined organic phases were dried over $\mathrm{MgSO}_{4}$ and evaporated under vacuum. The desired product $\mathbf{5 c}$ was obtained after column chromatography on silica gel (gradient from 0 to $15 \% \mathrm{DCM}: \mathrm{MeOH}$ ) as a brown oil (3.30 g, $11.4 \mathrm{mmol}, 84 \%$ ). IR (neat) $\mathbf{v}_{\max }=3411,3184$, 3052, 2922, 2849, 1437, 1345, 818, $753 \mathrm{~cm}^{-1} .{ }^{1} \mathrm{H}$ NMR (CDCl 3,500 MHz) $\delta=8.27(\mathrm{bs}, 1 \mathrm{H}), 7.87-7.76(\mathrm{~m}, 4 \mathrm{H}), 7.53(\mathrm{dd}, J=8.3,1.3 \mathrm{~Hz}$, $1 \mathrm{H}), 7.50-7.42(\mathrm{~m}, 2 \mathrm{H}), 7.33(\mathrm{~d}, J=8.1 \mathrm{~Hz}, 1 \mathrm{H}), 7.23-7.20(\mathrm{~m}, 1 \mathrm{H})$, $7.19(\mathrm{~d}, J=7.3 \mathrm{~Hz}, 1 \mathrm{H}), 7.14(\mathrm{~d}, J=7.0 \mathrm{~Hz}, 1 \mathrm{H}), 6.23(\mathrm{t}, J=2.1 \mathrm{~Hz}$, $1 \mathrm{H}), 4.16(\mathrm{~s}, 2 \mathrm{H}), 4.04(\mathrm{~s}, 2 \mathrm{H}), 2.08$ (bs, $2 \mathrm{H}) .{ }^{13} \mathrm{C}\left\{{ }^{1} \mathrm{H}\right\} \mathrm{NMR}\left(\mathrm{CDCl}_{3}\right.$, $75 \mathrm{MHz}) \delta=137.8\left(\mathrm{C}_{\mathrm{q}}\right), 136.1\left(\mathrm{C}_{\mathrm{q}}\right), 133.6\left(\mathrm{C}_{\mathrm{q}}\right), 132.7\left(\mathrm{C}_{\mathrm{q}}\right), 131.9\left(\mathrm{C}_{\mathrm{q}}\right)$, $128.2(\mathrm{CH}), 127.8(\mathrm{CH}), 127.7(\mathrm{CH}), 127.1\left(\mathrm{C}_{\mathrm{q}}\right), 126.8(\mathrm{CH}), 126.7$ $(\mathrm{CH}), 126.1(\mathrm{CH}), 125.6(\mathrm{CH}), 124.3(\mathrm{CH}), 122.0(\mathrm{CH}), 119.3(\mathrm{CH})$, $110.3(\mathrm{CH}), 100.5(\mathrm{CH}), 53.4\left(\mathrm{CH}_{2}\right), 51.1\left(\mathrm{CH}_{2}\right)$. HRMS (ESI) $\mathrm{m} / \mathrm{z}$ : calc for $\mathrm{C}_{20} \mathrm{H}_{18} \mathrm{~N}_{2}[\mathrm{M}+\mathrm{H}]^{+}$287.1548, found 287.1542.

\section{Scope of the 4-Iso Pictet-Spengler reaction}

General procedure 3 for the synthesis of tetrahydropyrrolo[4,3,2-de]isoquinolines 4: A mixture of tryptamine 5c (0.17 mmol), Cat b ( $2 \mathrm{~mol} \%, 0.0034 \mathrm{mmol}, 2.5 \mathrm{mg}$ ) and $3 \AA$ molecular sieves ( $82 \mathrm{mg}$ for $0.17 \mathrm{mmol}$ of $\mathbf{5 c}$, powdered) in dichloromethane $(2.4 \mathrm{~mL}$ for $0.17 \mathrm{mmol}$ of $\mathbf{5 c}$ ) was stirred for $5 \mathrm{~min}$ at room temperature under an argon atmosphere. Subsequently, aldehyde ( 2.0 equiv., $0.35 \mathrm{mmol}$ ) was added and the mixture stirred for $15 \mathrm{~h}$. The mixture was filtered under celite and silica was added. After evaporation of the volatiles, the silica mixture was purified by chromatography under silica gel to give the desired product 4. 3-(4-bromophenyl)-4-(naphthalen-2-ylmethyl)-1,3,4,5-tetrahydropyrrolo[4,3,2-de]isoquinoline $4 a$

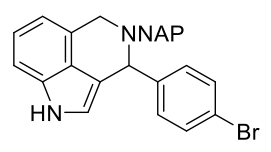

Compound 4a was synthesized following the General procedure 3 using tryptamine $5 c$ (50 mg, $0.17 \mathrm{mmol}, 1.0$ equiv.), 4-bromobenzaldehyde $(65 \mathrm{mg}, 0.35 \mathrm{mmol}$, 2.0 equiv.), Cat b $(2.5 \mathrm{mg}, 0.0034 \mathrm{mmol}, 2$ mol\%), M.S. $3 \AA ̊$ ( $82 \mathrm{mg})$, in DCM $(2.4 \mathrm{~mL})$. The desired product $4 \mathrm{a}$ was obtained after column chromatography on silica gel (gradient from 0 to $15 \%$ Heptane:EtOAc) as a white amorphous solid (58 $\mathrm{mg}, 0.13 \mathrm{mmol}, 75 \%)$.

$1 \mathrm{mmol}$ scale procedure: Compound 4a was synthesized following the General procedure 3 using tryptamine 5 c (300 mg, 1.05 mmol, 1.0 equiv.), 4-bromobenzaldehyde ( $387 \mathrm{mg}, 2.10 \mathrm{mmol}, 2.0$ equiv.), Cat b (15.5 mg, $0.021 \mathrm{mmol}, 2 \mathrm{~mol} \%)$, M.S. $3 \AA ̊$ (503 mg), in DCM $(15.0 \mathrm{~mL})$. The desired product was obtained after column chromatography on silica gel (gradient from 0 to $15 \%$ Heptane:EtOAc) as a white amorphous solid $(289 \mathrm{mg}, 0.64 \mathrm{mmol}, 61$ $\%)$. IR (neat) $v_{\max }=3406,3052,2959,2926,2838,1484,1264$, $1070,1010,817,752,735 \mathrm{~cm}^{-1} .{ }^{1} \mathbf{H}$ NMR $\left(\mathrm{CDCl}_{3}, 500 \mathrm{MHz}\right) \delta=8.00$ (bs, $1 \mathrm{H}), 7.85-7.77(\mathrm{~m}, 3 \mathrm{H}), 7,72(\mathrm{~s}, 1 \mathrm{H}), 7.59(\mathrm{~d}, J=8.4 \mathrm{~Hz}, 1 \mathrm{H})$, 7.50-7.44 (m, 2H), 7.43-7.40 (m, 2H), 7.24 (t, J=8.0 Hz, 1H), 7.25$7.21(\mathrm{~m}, 2 \mathrm{H}), 7.18(\mathrm{t}, J=7.0 \mathrm{~Hz}, 1 \mathrm{H}), 6.81(\mathrm{~d}, J=4.9 \mathrm{~Hz}, 1 \mathrm{H}), 6.75$ (s, $1 \mathrm{H}), 5.02(\mathrm{~s}, 1 \mathrm{H}), 4.03(\mathrm{~d}, J=15.8 \mathrm{~Hz}, 1 \mathrm{H}), 3.85(\mathrm{~d}, J=13.5 \mathrm{~Hz}$, $1 \mathrm{H}), 3.78(\mathrm{~d}, J=15.8 \mathrm{~Hz}, 1 \mathrm{H}), 3.69(\mathrm{~d}, J=13.5 \mathrm{~Hz}, 1 \mathrm{H}) .{ }^{13} \mathrm{C}\left\{{ }^{1} \mathrm{H}\right\}$ NMR $\left(\mathrm{CDCl}_{3}, 75 \mathrm{MHz}\right) \delta=141.8\left(\mathrm{C}_{\mathrm{q}}\right), 137.3\left(\mathrm{C}_{\mathrm{q}}\right), 133.5\left(\mathrm{C}_{\mathrm{q}}\right), 133.5\left(\mathrm{C}_{\mathrm{q}}\right)$, $132.9\left(\mathrm{C}_{\mathrm{q}}\right), 131.4(\mathrm{CH}), 130.5(\mathrm{CH}), 128.7\left(\mathrm{C}_{\mathrm{q}}\right), 128.1(\mathrm{CH}), 127.8$ $(\mathrm{CH}), 127.7(\mathrm{CH}), 127.4(\mathrm{CH}), 127.3(\mathrm{CH}), 126.0(\mathrm{CH}), 125.7(\mathrm{CH})$ $125.2\left(\mathrm{C}_{\mathrm{q}}\right), 123.2(\mathrm{CH}), 121.1\left(\mathrm{C}_{\mathrm{q}}\right), 119.3(\mathrm{CH}), 115.1(\mathrm{CH}), 113.3$ $\left(\mathrm{C}_{q}\right), 109.0(\mathrm{CH}), 60.9(\mathrm{CH}), 58.9\left(\mathrm{CH}_{2}\right), 49.4\left(\mathrm{CH}_{2}\right)$ HRMS (ESI): $\mathrm{m} / \mathrm{z}$ : calc for $\mathrm{C}_{27} \mathrm{H}_{22}{ }^{79} \mathrm{BrN}_{2}{ }^{+} 453.0966[\mathrm{M}+\mathrm{H}]^{+}$, found 453.0926, calc for $\mathrm{C}_{27} \mathrm{H}_{22}{ }^{81} \mathrm{BrN}_{2}{ }^{+} 455.0946[\mathrm{M}+\mathrm{H}]^{+}$, found 455.0925.

4-(naphthalen-2-ylmethyl)-3-phenyl-1,3,4,5-tetrahydropyrrolo[4,3,2-de]isoquinoline $4 \mathrm{~b}$

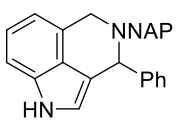

Compound $\mathbf{4 b}$ was synthesized following the General procedure 3 using tryptamine $5 c$ ( 50 $\mathrm{mg}, 0.17 \mathrm{mmol}, 1.0$ equiv.), benzaldehyde (37 $\mathrm{mg}, 0.35 \mathrm{mmol}, 2.0$ equiv.), Cat b $(2.5 \mathrm{mg}, 0.0034$ $\mathrm{mmol}, 2 \mathrm{~mol} \%)$, M.S. $3 \AA ̊(82 \mathrm{mg})$, in DCM $(2.4 \mathrm{~mL})$. The desired product $\mathbf{4 b}$ was obtained after column chromatography on silica gel (gradient from 0 to $15 \%$ Heptane:EtOAc) as a white amorphous solid (50 mg, $0.13 \mathrm{mmol}, 78 \%$ ). ${ }^{1} \mathbf{H}$ NMR (DMSO-d 6,500 MHz) $\delta=10.82(\mathrm{~s}, 1 \mathrm{H}), 7.91-7.83(\mathrm{~m}, 3 \mathrm{H}), 7.75(\mathrm{~s}, 1 \mathrm{H}), 7.56(\mathrm{~d}, J=$ $8.3 \mathrm{~Hz}, 2 \mathrm{H}), 7.50-7.44(\mathrm{~m}, 2 \mathrm{H}), 7.33-7.28(\mathrm{~m}, 4 \mathrm{H}), 7.27-7.19(\mathrm{~m}$, $2 \mathrm{H}), 7.03(\mathrm{t}, J=7.4 \mathrm{~Hz}, 1 \mathrm{H}), 6.92(\mathrm{~s}, 1 \mathrm{H}), 6.68(\mathrm{~d},=J=6.7 \mathrm{~Hz}, 1 \mathrm{H})$, $5.07(\mathrm{~s}, 1 \mathrm{H}), 3.85(\mathrm{~d}, J=5.6 \mathrm{~Hz}, 1 \mathrm{H}), 3.78-3.65(\mathrm{~m}, 3 \mathrm{H}) .{ }^{13} \mathrm{C}\left\{{ }^{1} \mathrm{H}\right\}$ NMR (DMSO-d $6,75 \mathrm{MHz}) \delta=142.5\left(\mathrm{C}_{\mathrm{q}}\right), 137.4\left(\mathrm{C}_{\mathrm{q}}\right), 133.2\left(\mathrm{C}_{\mathrm{q}}\right), 132.9$ $\left(\mathrm{C}_{\mathrm{q}}\right), 132.3\left(\mathrm{C}_{\mathrm{q}}\right), 128.2(\mathrm{CH}), 128.1(\mathrm{CH}), 127.8\left(\mathrm{C}_{\mathrm{q}}\right), 127.7(\mathrm{CH})$, $127.6(\mathrm{CH}), 127.5(\mathrm{CH}), 126.9(\mathrm{CH}), 126.8(\mathrm{CH}), 126.7(\mathrm{CH}), 126.0$ $(\mathrm{CH}), 125.5(\mathrm{CH}), 124.9\left(\mathrm{C}_{\mathrm{q}}\right), 121.9(\mathrm{CH}), 119.8(\mathrm{CH}), 113.6(\mathrm{CH})$, $112.2\left(\mathrm{C}_{\mathrm{q}}\right), 109.1(\mathrm{CH}), 61.0(\mathrm{CH}), 58.0\left(\mathrm{CH}_{2}\right), 48.5\left(\mathrm{CH}_{2}\right)$ HRMS (ESI): $\mathrm{m} / \mathrm{z}$ : calc for $\mathrm{C}_{27} \mathrm{H}_{23} \mathrm{~N}_{2}{ }^{+} 375.1861[\mathrm{M}+\mathrm{H}]^{+}$, found 375.1841 .

4-(naphthalen-2-ylmethyl)-3-(3-(trifluoromethyl)phenyl)-1,3,4,5tetrahydropyrrolo[4,3,2-de]isoquinoline 4c

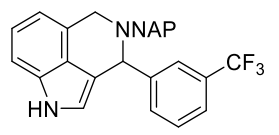

Compound $4 \mathrm{c}$ was synthesized following the General procedure 3 using tryptamine $5 \mathrm{c}(50 \mathrm{mg}, 0.17 \mathrm{mmol}, 1.0$ equiv.), 3-(trifluoromethyl)benzaldehyde (61 mg, $0.35 \mathrm{mmol}, 2.0$ equiv.), Cat b $(2.5 \mathrm{mg}, 0.0034 \mathrm{mmol} 2$ mol\%), M.S. $3 \AA ̊(82 \mathrm{mg})$, in DCM $(2.4 \mathrm{~mL})$. The desired product $4 \mathrm{c}$ was obtained after column chromatography on silica gel (gradient from 0 to $15 \%$ Heptane:EtOAc) as a white amorphous solid (58 
$\mathrm{mg}, 0.13 \mathrm{mmol}, 77 \%)$. IR (neat) $\mathbf{v}_{\max }=3419,3057,2927,1329$, 1164, 1123, 1072, $753 \mathrm{~cm}^{-1} .{ }^{1} \mathrm{H}_{\text {NMR }}\left(\mathrm{CDCl}_{3}, 500 \mathrm{MHz}\right) \delta=8.07$ (brs, $1 \mathrm{H}), 7.86-7.80(\mathrm{~m}, 2 \mathrm{H}), 7.80-7.76(\mathrm{~m}, 1 \mathrm{H}), 7.73(\mathrm{~s}, 1 \mathrm{H}), 7.69$ (s, 1H), $7.59(\mathrm{~d}, J=8.1 \mathrm{~Hz}, 1 \mathrm{H}), 7.55(\mathrm{~d}, J=8.1 \mathrm{~Hz}, 1 \mathrm{H}), 7.50(\mathrm{~d}, J=$ $7.8 \mathrm{~Hz}, 1 \mathrm{H}), 7.48-7.42(\mathrm{~m}, 1 \mathrm{H}), 7.40(\mathrm{t}, J=7.8 \mathrm{~Hz}, 1 \mathrm{H}), 7.29-7.24$ $(\mathrm{m}, 1 \mathrm{H}), 7.18(\mathrm{t}, J=7.5 \mathrm{~Hz}, 1 \mathrm{H}), 6.84-6.79(\mathrm{~m}, 2 \mathrm{H}), 5.10(\mathrm{~s}, 1 \mathrm{H}), 4.05$ $(\mathrm{d}, J=15.9 \mathrm{~Hz}, 1 \mathrm{H}), 3.86(\mathrm{~d}, J=13.5 \mathrm{~Hz}, 1 \mathrm{H}), 3.81(\mathrm{~d}, J=15.9 \mathrm{~Hz}$, $1 \mathrm{H}), 3.71(\mathrm{~d}, J=13.6 \mathrm{~Hz}, 1 \mathrm{H}) .{ }^{13} \mathrm{C}\left\{{ }^{1} \mathrm{H}\right\}$ NMR $\left(\mathrm{CDCl}_{3}, 75 \mathrm{MHz}\right) \delta=$ $144.0\left(C_{q}\right), 137.2\left(C_{q}\right), 133.6\left(C_{q}\right), 133.5\left(C_{q}\right), 133.0\left(C_{q}\right), 132.1(C H)$, $130.6\left(\mathrm{q}, \mathrm{C}-\mathrm{F},{ }^{2} \mathrm{~J}_{\mathrm{C}-\mathrm{F}}=32 \mathrm{~Hz}, \mathrm{C}_{\mathrm{q}}\right), 128.7(\mathrm{CH}), 128.6\left(\mathrm{C}_{\mathrm{q}}\right), 128.2(\mathrm{CH})$, $127.9(\mathrm{CH}), 127.8(\mathrm{CH}), 127.5(\mathrm{CH}), 127.3(\mathrm{CH}), 126.1(\mathrm{CH}), 125.7$ $(\mathrm{CH}), 125.5\left(\mathrm{q}, \mathrm{C}-\mathrm{F},{ }^{3} \mathrm{~J}_{\mathrm{C}-\mathrm{F}}=4 \mathrm{~Hz}, \mathrm{CH}\right), 125.1(\mathrm{C} q), 124.4\left(\mathrm{q}, \mathrm{C}-\mathrm{F},{ }^{1} \mathrm{~J}_{\mathrm{C}-\mathrm{F}}=\right.$ $272 \mathrm{~Hz}, \mathrm{Cq}), 124.1$ (q, $\left.J_{\mathrm{C}-\mathrm{F}}=4 \mathrm{~Hz}, \mathrm{CH}\right), 123.3(\mathrm{CH}), 119.6(\mathrm{CH}), 115.2$ $(\mathrm{CH}), 112.9\left(\mathrm{C}_{\mathrm{q}}\right), 61.0(\mathrm{CH}), 58.9\left(\mathrm{CH}_{2}\right), 49.5\left(\mathrm{CH}_{2}\right) .19 \mathrm{~F} \mathrm{NMR}\left(\mathrm{CDCl}_{3}\right.$, $282 \mathrm{MHz}) \delta=-62.3$. HRMS (ESI): $\mathrm{m} / \mathrm{z}$ : calc for $\mathrm{C}_{28} \mathrm{H}_{22} \mathrm{~F}_{3} \mathrm{~N}_{2}{ }^{+}$ $443.1735[\mathrm{M}+\mathrm{H}]^{+}$, found 443.1703 .

3-(2-fluorophenyl)-4-(naphthalen-2-ylmethyl)-1,3,4,5-tetrahydropyrrolo[4,3,2-de]isoquinoline 4d

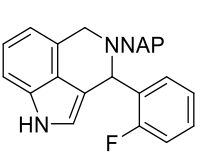

Compound 4d was synthesized following the General procedure 3 using tryptamine $\mathbf{5 c}$ (50 $\mathrm{mg}, 0.17 \mathrm{mmol}, 1.0$ equiv.), 2-fluorobenzaldehyde ( $43 \mathrm{mg}, 0.35 \mathrm{mmol}, 2.0$ equiv.), Cat b (6.5 $\mathrm{mg}, 0.0087 \mathrm{mmol} 5 \mathrm{~mol} \%)$, M.S. $3 \AA ̊(82 \mathrm{mg})$, in DCM (2.4 mL). The desired product $\mathbf{4 d}$ was obtained after column chromatography on silica gel (gradient from 0 to $15 \%$ Heptane:EtOAc) as a white amorphous solid ( $50 \mathrm{mg}, 0.13 \mathrm{mmol}, 73 \%$ ). IR (neat) $v_{\max }=3407,3055,2926,2851,1486,1452,1228,755$, $737 \mathrm{~cm}^{-1} .{ }^{1} \mathrm{H}$ NMR $\left(\mathrm{CDCl}_{3}, 500 \mathrm{MHz}\right) \delta=8.00$ (bs, $\left.1 \mathrm{H}\right), 7.87-7.79$ $(\mathrm{m}, 3 \mathrm{H}), 7,77(\mathrm{~s}, 1 \mathrm{H}), 7.59(\mathrm{~d}, J=8.3,1 \mathrm{H}), 7.51-7.44(\mathrm{~m}, 2 \mathrm{H}), 7.30-$ $7.23(\mathrm{~m}, 2 \mathrm{H}), 7.21(\mathrm{t}, J=7.5 \mathrm{~Hz}, 1 \mathrm{H}), 7.15(\mathrm{t}, J=9.2 \mathrm{~Hz}, 1 \mathrm{H}), 7.09(\mathrm{t}$, $J=7.2 \mathrm{~Hz}, 1 \mathrm{H}), 7.00(\mathrm{t}, J=7.4 \mathrm{~Hz}, 1 \mathrm{H}), 6.84(\mathrm{~d}, J=6.7 \mathrm{~Hz}, 1 \mathrm{H}), 6.74$ $(\mathrm{s}, 1 \mathrm{H}), 5.60(\mathrm{~s}, 1 \mathrm{H}), 4.08(\mathrm{~d}, J=15.8 \mathrm{~Hz}, 1 \mathrm{H}), 3.91(\mathrm{~d}, J=13.3 \mathrm{~Hz}$, $1 \mathrm{H}), 3.87-3.79(\mathrm{~m}, 2 \mathrm{H}) .{ }^{13} \mathrm{C}\left\{{ }^{1} \mathrm{H}\right\} \mathrm{NMR}\left(\mathrm{CDCl}_{3}, 75 \mathrm{MHz}\right) \delta=161.1(\mathrm{~d}$, $\left.C-F,{ }^{1} J_{C-F}=247 \mathrm{~Hz}, C q\right), 137.2\left(C_{q}\right), 133.5\left(C_{q}\right), 133.4\left(C_{q}\right), 132.9\left(C_{q}\right)$, 130.4 (d, C-F, $\left.{ }^{3} J_{C-F}=3.8 \mathrm{~Hz}, \mathrm{CH}\right), 129.7$ (d, C-F, $\left.{ }^{2} \mathrm{~J}_{\mathrm{C}-\mathrm{F}}=15.1 \mathrm{~Hz}, \mathrm{C}_{\mathrm{q}}\right)$, $128.9\left(\mathrm{~d}, \mathrm{C}-\mathrm{F},{ }^{3} \mathrm{~J}_{\mathrm{C}-\mathrm{F}}=8.8 \mathrm{~Hz}, \mathrm{CH}\right), 128.0(\mathrm{CH}), 127.9(\mathrm{CH}), 127.8(\mathrm{CH})$, $127.6(\mathrm{CH}), 127.5(\mathrm{CH}), 125.9(\mathrm{CH}), 125.7\left(\mathrm{C}_{\mathrm{q}}\right), 125.6(\mathrm{CH}), 123.9(\mathrm{~d}$, $\left.\mathrm{C}-\mathrm{F},{ }^{4} J_{\mathrm{C}-\mathrm{F}}=2.7 \mathrm{~Hz}, \mathrm{CH}\right), 123.2(\mathrm{CH}), 118.8(\mathrm{CH}), 115.5\left(\mathrm{~d}, \mathrm{C}-\mathrm{F},{ }^{2} J_{\mathrm{C}-\mathrm{F}}=\right.$ $22.5 \mathrm{~Hz}, \mathrm{CH}), 115.0(\mathrm{CH}), 113.5\left(\mathrm{C}_{\mathrm{q}}\right), 109.0(\mathrm{CH}), 59.0\left(\mathrm{CH}_{2}\right), 55.1$ (CH), $49.1\left(\mathrm{CH}_{2}\right) .{ }^{19} \mathrm{~F} \mathrm{NMR}\left(\mathrm{CDCl}_{3}, 282 \mathrm{MHz}\right) \delta=-118.0$. HRMS (ESI): $\mathrm{m} / \mathrm{z}$ : calc for $\mathrm{C}_{27} \mathrm{H}_{22} \mathrm{FN}_{2}{ }^{+} 393.1767[\mathrm{M}+\mathrm{H}]^{+}$, found 393.1756.

3-(4-methoxyphenyl)-4-(naphthalen-2-ylmethyl)-1,3,4,5-tetrahydropyrrolo[4,3,2-de]isoquinoline $4 \mathrm{e}$

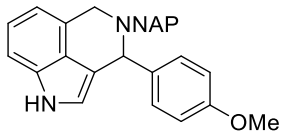

Compound $\mathbf{4 e}$ was synthesized following the General procedure 3 using tryptamine $5 \mathrm{c}(54 \mathrm{mg}, 0.19 \mathrm{mmol}, 1.0$ equiv.), 4-methoxybenzaldehyde $(51 \mathrm{mg}$, $0.38 \mathrm{mmol}, 2.0$ equiv.), Cat b (6.9 $\mathrm{mg}$,

$0.0094 \mathrm{mmol}, 5 \mathrm{~mol} \%)$, M.S. $3 \AA$ (91 mg), in DCM $(2.7 \mathrm{~mL})$. The desired product $4 \mathbf{e}$ was obtained after column chromatography on silica gel (gradient from 0 to $15 \%$ Heptane:EtOAc) as a white amorphous solid ( $29 \mathrm{mg}, 0.07 \mathrm{mmol}, 38 \%$ ). IR (neat) $\mathbf{v}_{\max }=3402$, 3055, 2930, 2836, 1607, 1508, 1451, 1248, 1172, 1031, 819, 753 $\mathrm{cm}^{-1} .{ }^{1} \mathrm{H} \mathrm{NMR}\left(\mathrm{CDCl}_{3}, 500 \mathrm{MHz}\right) \delta=8.01(\mathrm{~s}, 1 \mathrm{H}), 7.86-7.78(\mathrm{~m}, 3 \mathrm{H})$, $7.75(\mathrm{~s}, 1 \mathrm{H}), 7.60(\mathrm{~d}, J=8.3 \mathrm{~Hz}, 1 \mathrm{H}), 7.49-7.43(\mathrm{~m}, 2 \mathrm{H}), 7.30-7.23$ $(\mathrm{m}, 3 \mathrm{H}), 7.18(\mathrm{t}, J=7.3 \mathrm{~Hz}, 1 \mathrm{H}), 6.86(\mathrm{~d}, J=8.8 \mathrm{~Hz}, 2 \mathrm{H}), 6.81(\mathrm{~d}, J=$ $7.0 \mathrm{~Hz}, 1 \mathrm{H}), 6.77(\mathrm{~s}, 1 \mathrm{H}), 5.05(\mathrm{~s}, 1 \mathrm{H}), 4.08(\mathrm{~d}, J=15.7 \mathrm{~Hz}, 1 \mathrm{H}), 3.89$ (d, $J=13.6 \mathrm{~Hz}, 1 \mathrm{H}), 3.83-3.77(\mathrm{~m}, 1 \mathrm{H}), 3.80(\mathrm{~s}, 3 \mathrm{H}), 3.70(\mathrm{~d}, J=14.0$ $\mathrm{Hz}, 1 \mathrm{H}){ }^{13} \mathrm{C}\left\{{ }^{1} \mathrm{H}\right\}$ NMR $\left(\mathrm{CDCl}_{3}, 75 \mathrm{MHz}\right) \delta=206.3\left(\mathrm{C}_{\mathrm{q}}\right), 158.9\left(\mathrm{C}_{\mathrm{q}}\right)$, $137.7\left(C_{q}\right), 134.8\left(C_{q}\right), 133.6\left(C_{q}\right), 133.5\left(C_{q}\right), 132.9\left(C_{q}\right), 129.9(C H)$, $129.1\left(\mathrm{C}_{\mathrm{q}}\right), 128.0(\mathrm{CH}), 127.9(\mathrm{CH}), 127.8(\mathrm{CH}), 127.5(\mathrm{CH}), 127.4$ $(\mathrm{CH}), 126.0(\mathrm{CH}), 125.6(\mathrm{CH}), 125.5\left(\mathrm{C}_{\mathrm{q}}\right), 123.1(\mathrm{CH}), 119.2(\mathrm{CH})$,
$115.0(\mathrm{CH}), 113.7(\mathrm{CH}), 108.9(\mathrm{CH}), 61.2(\mathrm{CH}), 58.7\left(\mathrm{CH}_{2}\right), 55.4$ $\left(\mathrm{CH}_{3}\right), 49.6\left(\mathrm{CH}_{2}\right)$ HRMS (ESI): $\mathrm{m} / z$ : calc for $\mathrm{C}_{28} \mathrm{H}_{25} \mathrm{~N}_{2} \mathrm{O}^{+} 405.1967$ $[\mathrm{M}+\mathrm{H}]^{+}$, found 405.1947 .

3-(4-(naphthalen-2-ylmethyl)-1,3,4,5-tetrahydropyrrolo[4,3,2de] isoquinolin-3-yl)benzaldehyde $\mathbf{4 f}$

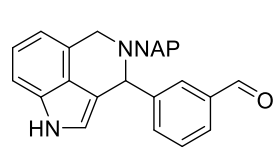

Compound $\mathbf{4 f}$ was synthesized following the General procedure 3 using tryptamine $5 c$ (76 mg, $0.27 \mathrm{mmol}, 1.0$ equiv.), isophthalaldehyde $(71 \mathrm{mg}, 0.53 \mathrm{mmol}, 2.0$ equiv.), Cat b (3.9 mg, $0.0053 \mathrm{mmol}, 2$ mol\%), M.S. $3 \AA ̊(130 \mathrm{mg})$, in DCM (3.8 mL). The desired product $4 \mathrm{f}$ was obtained after column chromatography on silica gel (gradient from 0 to $15 \%$ Heptane:EtOAc) as a white amorphous solid (50 $\mathrm{mg}, 0.12 \mathrm{mmol}, 46 \%$ ). IR (neat) $\mathbf{v}_{\max }=3406,3054,2925,3838$, 1693, 1600, 1584, 1445, 1342, 1146, 819, 754, $736 \mathrm{~cm}^{-1} .{ }^{1} \mathrm{H}$ NMR $\left(\mathrm{CDCl}_{3}, 300 \mathrm{MHz}\right) \delta=9.97(\mathrm{~s}, 1 \mathrm{H}), 8.12(\mathrm{bs}, 1 \mathrm{H}), 7.89-7.82(\mathrm{~m}, 3 \mathrm{H})$, 7.81-7.76 (m, 2H), $7.73(\mathrm{~s}, 1 \mathrm{H}), 7.69(\mathrm{~d}, J=7.5 \mathrm{~Hz}, 1 \mathrm{H}), 7.61$ (dd, $J$ $=8.3,1.8 \mathrm{~Hz}, 1 \mathrm{H}), 7.50-7.42(\mathrm{~m}, 3 \mathrm{H}), 7.29(\mathrm{~d}, J=7.7 \mathrm{~Hz}, 1 \mathrm{H}), 7.20$ $(\mathrm{t}, J=7.5 \mathrm{~Hz}, 1 \mathrm{H}), 6.83-6.81(\mathrm{~m}, 2 \mathrm{H}), 5.16(\mathrm{~s}, \mathrm{H}), 4.05(\mathrm{~d}, J=15.7$ $\mathrm{Hz}, 1 \mathrm{H}), 3.87(\mathrm{~d}, J=13.4 \mathrm{~Hz}, 1 \mathrm{H}), 3.82(\mathrm{~d}, J=16.0 \mathrm{~Hz}, 1 \mathrm{H}), 3.75(\mathrm{~d}$, $J=13.3 \mathrm{~Hz}, 1 \mathrm{H}) .{ }^{13} \mathrm{C}\left\{{ }^{1} \mathrm{H}\right\} \mathrm{NMR}\left(\mathrm{CDCl}_{3}, 75 \mathrm{MHz}\right) \delta=192.7(\mathrm{CH}), 144.2$ $(\mathrm{Cq}), 137.2(\mathrm{Cq}), 136.6(\mathrm{Cq}), 135.0(\mathrm{CH}), 133.7(\mathrm{Cq}), 133.5(\mathrm{Cq})$, $133.0(\mathrm{Cq}), 130.4(\mathrm{CH}), 129.1(\mathrm{CH}), 128.5(\mathrm{CH}), 128.2(\mathrm{CH}), 127.8$ $(\mathrm{CH}), 127.6(\mathrm{CH}), 127.3(\mathrm{CH}), 126.1(\mathrm{CH}), 125.7(\mathrm{CH}), 125.1(\mathrm{Cq})$, $123.3(\mathrm{CH}), 119.7(\mathrm{CH}), 115.2(\mathrm{CH}), 112.7(\mathrm{Cq}), 109.2(\mathrm{CH}), 60.8$ (CH), $58.9\left(\mathrm{CH}_{2}\right), 49.3\left(\mathrm{CH}_{2}\right)$. HRMS (ESI): $\mathrm{m} / \mathrm{z}$ : calcd for $\mathrm{C}_{28} \mathrm{H}_{23} \mathrm{~N}_{2} \mathrm{O}^{+}$ 403.1810 $[\mathrm{M}+\mathrm{H}]^{+}$, found 403.1797.

4-(naphthalen-2-ylmethyl)-3-(4-(4,4,5,5-tetramethyl-1,3,2-dioxaborolan-2-yl)phenyl)-1,3,4,5-tetrahydropyrrolo[4,3,2-de]isoquinoline $\mathbf{4 g}$

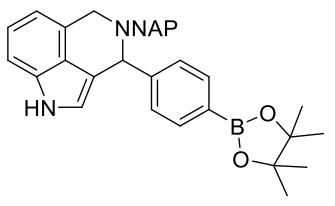

Compound $\mathbf{4 g}$ was synthesized following the General procedure 3 using tryptamine $5 \mathrm{c}(50 \mathrm{mg}, 0.17$ mmol, 1.0 equiv. $), 4-(4,4,5,5$-tetramethyl-1,3,2-dioxaborolan-2yl)benzaldehyde ( $80 \mathrm{mg}, 0.35 \mathrm{mmol}$, 2.0 equiv.), Cat b ( $2.6 \mathrm{mg}, 0.0034 \mathrm{mmol}, 2 \mathrm{~mol} \%)$, M.S. $3 \AA$ ( $82 \mathrm{mg}$ ), in DCM $(2.5 \mathrm{~mL})$. The desired product $\mathbf{4 g}$ was obtained after column chromatography on silica gel (gradient from 0 to $15 \%$ Heptane:EtOAc) as a white amorphous solid ( $46 \mathrm{mg}, 0.09 \mathrm{mmol}, 53 \%$ ). IR (neat) $v_{\max }=3362,3053,2977,2932,1604,1449,1397,1359$, $1322,1143,1087,858,750 \mathrm{~cm}^{-1}$. ${ }^{1} \mathbf{H}$ NMR $\left(\mathrm{CDCl}_{3}, 300 \mathrm{MHz}\right) \delta=$ 7.99 (brs, $1 \mathrm{H}), 7.86-7.73(\mathrm{~m}, 6 \mathrm{H}), 7.57$ (dd, $J=1.5 ; 8.4 \mathrm{~Hz}, 1 \mathrm{H}), 7.48$ $7.39(\mathrm{~m}, 4 \mathrm{H}), 7.24(\mathrm{~d}, J=9.3 \mathrm{~Hz}, 1 \mathrm{H}), 7.16(\mathrm{dd}, J=6.8 ; 8.3 \mathrm{~Hz}, 1 \mathrm{H})$, $6.78(\mathrm{~d}, J=6.9 \mathrm{~Hz}, 1 \mathrm{H}), 6.70(\mathrm{~d}, J=1.2 \mathrm{~Hz}, 1 \mathrm{H}), 5.06(\mathrm{~s}, 1 \mathrm{H}), 4.08$ $(d, J=15.6 \mathrm{~Hz}, 1 \mathrm{H}), 3.92(\mathrm{~d}, J=13.4 \mathrm{~Hz}, 1 \mathrm{H}), 3.79(\mathrm{~d}, J=15.6 \mathrm{~Hz}$, $1 \mathrm{H}), 3.66(\mathrm{~d}, J=13.4 \mathrm{~Hz}, 1 \mathrm{H}), 1.34(\mathrm{~s}, 12 \mathrm{H}) .{ }^{13} \mathrm{C}\left\{{ }^{1} \mathrm{H}\right\}$ NMR $\left(\mathrm{CDCl}_{3}, 75\right.$ MHz) $\delta=146.1\left(\mathrm{C}_{\mathrm{q}}\right), 137,6\left(\mathrm{C}_{\mathrm{q}}\right), 134.9(\mathrm{CH}), 133.5\left(\mathrm{C}_{\mathrm{q}}\right), 132.9\left(\mathrm{C}_{\mathrm{q}}\right)$, $129.5\left(\mathrm{C}_{\mathrm{q}}\right), 129.1\left(\mathrm{C}_{\mathrm{q}}\right), 128.3(2 \mathrm{CH}), 128.0(\mathrm{CH}), 127.8(\mathrm{CH}), 127.8$ $(\mathrm{CH}), 127.5(\mathrm{CH}), 127.4(\mathrm{CH}), 126.0\left(\mathrm{C}_{\mathrm{q}}\right), 126.0(\mathrm{CH}), 125.6(\mathrm{CH})$, $125.4\left(\mathrm{C}_{\mathrm{q}}\right), 123.1(\mathrm{CH}), 119.2(\mathrm{CH}), 114.9(\mathrm{CH}), 114.8\left(\mathrm{C}_{\mathrm{q}}\right), 108.9$ $(\mathrm{CH}), 83.9\left(\mathrm{C}_{\mathrm{q}}\right), 62.4(\mathrm{CH}), 59.0\left(\mathrm{CH}_{2}\right), 50.1\left(\mathrm{CH}_{2}\right), 25.0\left(\mathrm{CH}_{3}\right) .{ }^{11} \mathrm{~B}$ NMR ( $\left.\mathrm{CDCl}_{3}, 160 \mathrm{MHz}\right) \delta=22.45$. HRMS (ESI): $\mathrm{m} / \mathrm{z}$ : calcd for $\mathrm{C}_{33} \mathrm{H}_{34} \mathrm{BN}_{2} \mathrm{O}_{2}{ }^{+}$501.2713 [M+H] $]^{+}$, found 501.2689.

4-(naphthalen-2-ylmethyl)-3-(quinolin-4-yl)-1,3,4,5-tetrahydropyrrolo[4,3,2-de]isoquinoline $\mathbf{4 h}$

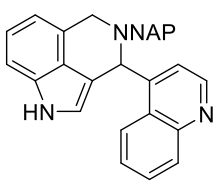

Compound $\mathbf{4 h}$ was synthesized following the General procedure $\mathbf{3}$ using tryptamine $\mathbf{5 c}$ (50 $\mathrm{mg}, 0.17 \mathrm{mmol}, 1.0$ equiv.), quinoline-4carbaldehyde ( $55 \mathrm{mg}, 0.35 \mathrm{mmol}, 2.0$ equiv.), Cat b $(2.6 \mathrm{mg}, 0.0034 \mathrm{mmol}, 2 \mathrm{~mol} \%)$, M.S. 3 
$\AA$ ( $82 \mathrm{mg})$, in DCM $(2.5 \mathrm{~mL})$. The desired product $4 \mathrm{~h}$ was obtained after column chromatography on silica gel (gradient from 0 to 15 \% Heptane:EtOAc) as a white amorphous solid (53 mg, $0.12 \mathrm{mmol}$, $71 \%)$. IR (neat) $v_{\max }=3054,2839,1590,1508,1445,1345,818$, $757 \mathrm{~cm}^{-1} .{ }^{1} \mathrm{H} \mathrm{NMR}\left(\mathrm{CDCl}_{3}, 500 \mathrm{MHz}\right) \delta=8.76(\mathrm{~d}, J=4.4 \mathrm{~Hz}, 1 \mathrm{H}), 8.52$ (brs, $1 \mathrm{H}$ ), 8.30 (d, $J=8.5 \mathrm{~Hz}, 1 \mathrm{H}), 8.18(\mathrm{~d}, J=8.4 \mathrm{~Hz}, 1 \mathrm{H}), 7.88-7.78$ $(\mathrm{m}, 3 \mathrm{H}), 7.75-7.70(\mathrm{~m}, 2 \mathrm{H}), 7.52(\mathrm{~d}, J=8.4 \mathrm{~Hz}, 1 \mathrm{H}), 7.51-7.44(\mathrm{~m}$, $3 \mathrm{H}), 7.31(\mathrm{~d}, J=8.1 \mathrm{~Hz}, 1 \mathrm{H}), 7.23(\mathrm{t}, J=7.5 \mathrm{~Hz}, 1 \mathrm{H}), 7.11(\mathrm{~d}, J=4.3$ $\mathrm{Hz}, 1 \mathrm{H}), 6.84(\mathrm{~d}, J=7.0 \mathrm{~Hz}, 1 \mathrm{H}), 6.75(\mathrm{~s}, 1 \mathrm{H}), 5.79(\mathrm{~s}, 1 \mathrm{H}), 4.05$ (d, J $=16.0 \mathrm{~Hz}, 1 \mathrm{H}), 4.02(\mathrm{~d}, J=14.2 \mathrm{~Hz}, 1 \mathrm{H}), 3.80(\mathrm{~d}, J=15 . .9 \mathrm{~Hz}, 1 \mathrm{H})$, $3.73(\mathrm{~d}, J=14.2 \mathrm{~Hz}, 1 \mathrm{H}),{ }^{13} \mathrm{C}\left\{{ }^{1} \mathrm{H}\right\} \mathrm{NMR}\left(\mathrm{CDCl}_{3}, 75 \mathrm{MHz}\right) \delta=150.1$ $(\mathrm{CH}), 148.9\left(\mathrm{C}_{\mathrm{q}}\right), 148.5\left(\mathrm{C}_{\mathrm{q}}\right), 136.8\left(\mathrm{C}_{\mathrm{q}}\right), 133.6\left(\mathrm{C}_{\mathrm{q}}\right), 133.4\left(\mathrm{C}_{\mathrm{q}}\right), 133.0$ $\left(\mathrm{C}_{\mathrm{q}}\right), 129.9(\mathrm{CH}), 129.2(\mathrm{CH}), 128.2\left(\mathrm{C}_{\mathrm{q}}\right), 128.1(\mathrm{CH}), 127.9(\mathrm{CH})$, $127.8(\mathrm{CH}), 127.8(\mathrm{CH}), 127.7(\mathrm{CH}), 127.3\left(\mathrm{C}_{\mathrm{q}}\right), 126.3(\mathrm{CH}), 126.1$ $(\mathrm{CH}), 125.8(\mathrm{CH}), 125.4(\mathrm{CH}), 125.4\left(\mathrm{C}_{\mathrm{q}}\right), 123.4(\mathrm{CH}), 121.4(\mathrm{CH})$, $119.6(\mathrm{CH}), 115.3(\mathrm{CH}), 112.0\left(\mathrm{C}_{\mathrm{q}}\right), 109.3(\mathrm{CH}), 59.4\left(\mathrm{CH}_{2}\right), 59.2$ (CH), $49.5\left(\mathrm{CH}_{2}\right)$. HRMS (ESI): $\mathrm{m} / \mathrm{z}$ : calc for $\mathrm{C}_{30} \mathrm{H}_{24} \mathrm{~N}_{3}{ }^{+} 426.1970$ $[\mathrm{M}+\mathrm{H}]^{+}$, found 426.1932 .

4-(naphthalen-2-ylmethyl)-3-phenethyl-1,3,4,5-tetrahydropyrrolo[4,3,2-de]isoquinoline 4i

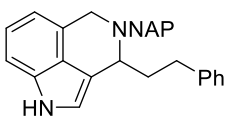

Compound $4 \mathbf{i}$ was synthesized following the General procedure 3 using tryptamine $5 c$ (50mg, $0.17 \mathrm{mmol}, 1.0$ equiv.), 3-phenylpropanal (47 mg, $0.35 \mathrm{mmol}, 2.0$ equiv.), Cat b (6.5 mg, $0.0087 \mathrm{mmol}, 5 \mathrm{~mol} \%)$, M.S. $3 \AA ̊$ ( $82 \mathrm{mg}$ ), in DCM (2.5 mL). The desired product $\mathbf{4} \mathbf{i}$ was obtained after column chromatography on silica gel (gradient from 0 to $15 \%$ Heptane:EtOAc) as an oil (29 mg, $0.12 \mathrm{mmol}, 75 \%$ ). IR (neat) $\mathbf{v}_{\max }=3057,2928,2852$, $1602,1495,1452,820,751,699 \mathrm{~cm}^{-1} .{ }^{1} \mathrm{H} \mathrm{NMR}\left(\mathrm{CDCl}_{3}, 300 \mathrm{MHz}\right) \delta$ $=7.98(\mathrm{brs}, 1 \mathrm{H}), 7.92-7.80(\mathrm{~m}, 3 \mathrm{H}), 7.72(\mathrm{~s}, 1 \mathrm{H}), 7.62(\mathrm{dd}, J=1.9$, 8.5 Hz, $1 \mathrm{H}), 7.56-7.45(\mathrm{~m}, 2 \mathrm{H}), 7-35-7.27(\mathrm{~m}, 2 \mathrm{H}), 7.25-7.15(\mathrm{~m}$, $5 \mathrm{H}), 6.95(\mathrm{~d}, J=2.1 \mathrm{~Hz}, 1 \mathrm{H}), 6.88(\mathrm{~d}, J=7.0 \mathrm{~Hz}, 1 \mathrm{H}), 4.39$ (d, $J=16.4$ $\mathrm{Hz}, 1 \mathrm{H}), 4.10(\mathrm{t}, J=7.5 \mathrm{~Hz}, 1 \mathrm{H}), 3.94(\mathrm{~d}, J=16.6 \mathrm{~Hz}, 1 \mathrm{H}), 3.82(\mathrm{~d}, J$ $=13.6 \mathrm{~Hz}, 1 \mathrm{H}), 3.71(\mathrm{~d}, J=13.6 \mathrm{~Hz}, 1 \mathrm{H}), 3.01-2.82(\mathrm{~m}, 2 \mathrm{H}), 2.39-$ $2.17(\mathrm{~m}, 1 \mathrm{H}), 2.07-1.90(\mathrm{~m}, 1 \mathrm{H}) .{ }^{13} \mathrm{C}\left\{{ }^{1} \mathrm{H}\right\} \mathrm{NMR}\left(\mathrm{CDCl}_{3}, 75 \mathrm{MHz}\right) \delta=$ $142.5\left(C_{q}\right), 133.7\left(C_{q}\right), 133.4\left(C_{q}\right), 132.8\left(C_{q}\right), 128.5(C H), 128.4\left(C_{q}\right)$, $128.4\left(\mathrm{C}_{\mathrm{q}}\right), 128.3(\mathrm{CH}), 127.8(\mathrm{CH}), 127.8(\mathrm{CH}), 127.6(\mathrm{CH}), 127.5$ $(\mathrm{CH}), 127.4(\mathrm{CH}), 125.8(\mathrm{CH}), 125.6(\mathrm{CH}), 125.4(\mathrm{CH}), 123.0(\mathrm{CH})$, $118.3\left(\mathrm{C}_{\mathrm{q}}\right), 118.2(\mathrm{CH}), 115.2(\mathrm{CH}), 114.1\left(\mathrm{C}_{\mathrm{q}}\right), 108.8(\mathrm{CH}), 58.1$ $\left(\mathrm{CH}_{2}\right), 56.8(\mathrm{CH}), 47.6\left(\mathrm{CH}_{2}\right), 36.7\left(\mathrm{CH}_{2}\right), 32.5\left(\mathrm{CH}_{2}\right)$. HRMS (ESI): $\mathrm{m} / \mathrm{z}$ : calc for $\mathrm{C}_{29} \mathrm{H}_{27} \mathrm{~N}_{2}+403.2174[\mathrm{M}+\mathrm{H}]^{+}$, found 403.2174 .

3-isobutyl-4-(naphthalen-2-ylmethyl)-1,3,4,5-tetrahydropyrrolo[4,3,2-de]isoquinoline $\mathbf{4 j}$

Compound $\mathbf{4} \mathbf{j}$ was synthesized following the Gen-<smiles>CC(C)CC1c2c[nH]c3cccc(c23)CN1[P+]</smileseral procedure 3 using tryptamine $5 \mathrm{c}(35 \mathrm{mg}, 0.12$ mmol, 1.0 equiv.), 3-methylbutanal $(21 \mathrm{mg}, 0.24$ mmol, 2.0 equiv.), Cat b (1.8 mg, $0.0024 \mathrm{mmol}, 2$ mol\%), M.S. $3 \AA$ A $(58 \mathrm{mg})$, in DCM (1.8 mL). The desired product 4 j was obtained after column chromatography on silica gel (gradient from 0 to $15 \%$ Heptane:EtOAc) as an oil (30 mg, $0.08 \mathrm{mmol}, 69$ \%). IR (neat) $v_{\max }=3414,3054,2952,2928,2866,1444,821,751$ $\mathrm{cm}^{-1} .{ }^{1} \mathrm{H}$ NMR $\left(\mathrm{CDCl}_{3}, \mathbf{5 0 0} \mathrm{MHz}\right) \delta=7.93$ (brs, $\left.1 \mathrm{H}\right)$, 7.86-7.77 (m, $3 \mathrm{H}), 7.67(\mathrm{~s}, 1 \mathrm{H}), 7.56(\mathrm{~d}, J=7.2 \mathrm{~Hz}, 1 \mathrm{H}), 7.48-7.43(\mathrm{~m}, 2 \mathrm{H}), 7.26$ $(\mathrm{d}, J=8.1 \mathrm{~Hz}, 1 \mathrm{H}), 7.20(\mathrm{dd}, J=1.0,8.0 \mathrm{~Hz}, 1 \mathrm{H}), 6.89(\mathrm{~d}, J=1.4 \mathrm{~Hz}$, $1 \mathrm{H}), 6.84(\mathrm{~d}, J=6.9 \mathrm{~Hz}, 1 \mathrm{H}), 4.31(\mathrm{~d}, J=16.4 \mathrm{~Hz}, 1 \mathrm{H}), 4.14(\mathrm{t}, J=7.5$ $\mathrm{Hz}, 1 \mathrm{H}), 3.86(\mathrm{~d}, J=16.4 \mathrm{~Hz}, 1 \mathrm{H}), 3.77(\mathrm{~d}, J=13.4 \mathrm{~Hz}, 1 \mathrm{H}), 3.66$ (d, $J=13.5 \mathrm{~Hz}, 1 \mathrm{H}), 1.98(\mathrm{sept}, J=6.8 \mathrm{~Hz}, 1 \mathrm{H}), 1.85-1.76(\mathrm{~m}, 1 \mathrm{H}), 1.56-$ $1.48(\mathrm{~m}, 1 \mathrm{H}), 0.98(\mathrm{~d}, J=6.7 \mathrm{~Hz}, 1 \mathrm{H}), 0.91(\mathrm{~d}, J=6.6 \mathrm{~Hz}, 1 \mathrm{H}) .{ }^{13} \mathrm{C}\left\{{ }^{1} \mathrm{H}\right\}$ NMR $\left(\mathrm{CDCl}_{3}, 75 \mathrm{MHz}\right) \delta=138.0\left(\mathrm{C}_{\mathrm{q}}\right), 133.8\left(\mathrm{C}_{\mathrm{q}}\right), 133.5\left(\mathrm{C}_{\mathrm{q}}\right), 132.9$ $\left(\mathrm{C}_{\mathrm{q}}\right), 128.4\left(\mathrm{C}_{\mathrm{q}}\right), 127.8(\mathrm{CH}), 127.8(\mathrm{CH}), 127.6(\mathrm{CH}), 127.4(\mathrm{CH})$, $125.9(\mathrm{CH}), 125.5(\mathrm{CH}), 124.9\left(\mathrm{C}_{\mathrm{q}}\right), 123.1(\mathrm{CH}), 118.1(\mathrm{CH}), 115.2$ $(\mathrm{CH}), 114.6\left(\mathrm{C}_{\mathrm{q}}\right), 108.8(\mathrm{CH}), 58.3\left(\mathrm{CH}_{2}\right), 55.4(\mathrm{CH}), 47.5\left(\mathrm{CH}_{2}\right), 44.3$
$\left(\mathrm{CH}_{2}\right), 24.5(\mathrm{CH}), 23.0\left(\mathrm{CH}_{3}\right), 22.9\left(\mathrm{CH}_{3}\right)$. HRMS (ESI): $\mathrm{m} / \mathrm{z}$ : calc for $\mathrm{C}_{25} \mathrm{H}_{27} \mathrm{~N}_{2}+355.2174[\mathrm{M}+\mathrm{H}]^{+}$, found 355.2143.

3-(but-3-en-1-yl)-4-(naphthalen-2-ylmethyl)-1,3,4,5-tetrahydropyrrolo[4,3,2-de]isoquinoline $\mathbf{4 k}$

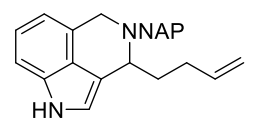

Compound $\mathbf{4 k}$ was synthesized following the General procedure 3 using tryptamine 5c (50mg, $0.17 \mathrm{mmol}, 1.0$ equiv.), hex-5enal ( $29 \mathrm{mg}, 0.35 \mathrm{mmol}, 2.0$ equiv.), Cat $\mathbf{b}$ (2.6 mg, $0.0035 \mathrm{mmol}, 2 \mathrm{~mol} \%$ ), M.S. $3 \AA$ ( $82 \mathrm{mg}$ ), in DCM (2.5 mL). The desired product $\mathbf{4 k}$ was obtained after column chromatography on silica gel (gradient from 0 to $15 \%$ Heptane:EtOAc) as an oil (32 mg, $0.09 \mathrm{mmol}, 53 \%$ ). IR (neat) $\boldsymbol{v}_{\max }=3411,3054,2974$, 2929, 2848, 1443, 1343, 1092, 909, 816, 750, $738 \mathrm{~cm}^{-1} .{ }^{1} \mathbf{H}$ NMR $\left(\mathrm{CDCl}_{3}, 500 \mathrm{MHz}\right) \delta=7.94$ (brs, $\left.1 \mathrm{H}\right), 7.87-7.77(\mathrm{~m}, 3 \mathrm{H}), 7.67(\mathrm{~s}, 1 \mathrm{H})$, $7.57(\mathrm{~d}, J=8.2 \mathrm{~Hz}, 1 \mathrm{H}), 7.50-7.43(\mathrm{~m}, 2 \mathrm{H}), 7.26(\mathrm{~d}, J=8.1 \mathrm{~Hz}, 1 \mathrm{H})$, $7.20(\mathrm{dd}, J=0.9 ; 7.0 \mathrm{~Hz}, 1 \mathrm{H}), 6.90(\mathrm{~d}, J=1.2 \mathrm{~Hz}, 1 \mathrm{H}), 6.84(\mathrm{~d}, J=$ $6.8 \mathrm{~Hz}, 1 \mathrm{H}$ ), 5.87 (tdd, $J=6.7,10.2,17.1 \mathrm{~Hz}, 1 \mathrm{H}$ ), 5.05 (dd, $J=2.1$, $17.2 \mathrm{~Hz}, 1 \mathrm{H}), 4.95(\mathrm{~d}, J=9.9 \mathrm{~Hz}, 1 \mathrm{H}), 4.33(\mathrm{~d}, J=16.3 \mathrm{~Hz}, 1 \mathrm{H}), 4.04$ $(\mathrm{t}, J=7.6 \mathrm{~Hz}, 1 \mathrm{H}), 3.87(\mathrm{~d}, J=16.5 \mathrm{~Hz}, 1 \mathrm{H}), 3.77(\mathrm{~d}, J=13.5 \mathrm{~Hz}, 1 \mathrm{H})$, $3.68(\mathrm{~d}, J=14.0 \mathrm{~Hz}, 1 \mathrm{H}), 2.38-2.24(\mathrm{~m}, 2 \mathrm{H}), 2.07-1.98(\mathrm{~m}, 1 \mathrm{H}), 1.78-$ $1.69(\mathrm{~m}, 1 \mathrm{H}) .{ }^{13} \mathrm{C}\left\{{ }^{1} \mathrm{H}\right\}$ NMR $\left(\mathrm{CDCl}_{3}, 75 \mathrm{MHz}\right) \delta=138.9(\mathrm{CH}), 137.8$ $\left(\mathrm{C}_{\mathrm{q}}\right), 133.8\left(\mathrm{C}_{\mathrm{q}}\right), 133.5\left(\mathrm{C}_{\mathrm{q}}\right), 132.9\left(\mathrm{C}_{\mathrm{q}}\right), 128.3\left(\mathrm{C}_{\mathrm{q}}\right), 127.9(\mathrm{CH}), 127.9$ $(\mathrm{CH}), 127.8(\mathrm{CH}), 127.6(\mathrm{CH}), 127.4(\mathrm{CH}), 125.9(\mathrm{CH}), 125.5(\mathrm{CH})$, $124.8\left(\mathrm{C}_{\mathrm{q}}\right), 123.1(\mathrm{CH}), 118.4(\mathrm{CH}), 115.3(\mathrm{CH}), 114.7\left(\mathrm{CH}_{2}\right), 114.2$ $\left(\mathrm{C}_{\mathrm{q}}\right), 108.9(\mathrm{CH}), 58.4\left(\mathrm{CH}_{2}\right), 57.0(\mathrm{CH}), 47.6\left(\mathrm{CH}_{2}\right), 34.2\left(\mathrm{CH}_{2}\right), 30.5$ $\left(\mathrm{CH}_{2}\right)$. HRMS (ESI): $\mathrm{m} / \mathrm{z}$ : calc for $\mathrm{C}_{25} \mathrm{H}_{25} \mathrm{~N}_{2}{ }^{+} 353.2017[\mathrm{M}+\mathrm{H}]^{+}$, found 353.2031.

\section{$\mathrm{C} 4 / \mathrm{N}$-Iso Pictet-Spengler reaction cascades} Synthesis of tryptamine $\mathbf{5 d}$.

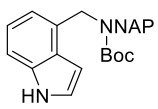

$\mathrm{Et}_{3} \mathrm{~N}$ (3.6 mL, $25.9 \mathrm{mmol}, 5.0$ equiv.) was added to a mixture of tryptamine $5 \mathrm{c}(1.50 \mathrm{~g}, 5.18 \mathrm{mmol}, 1.0$ equiv.) and $\mathrm{Boc}_{2} \mathrm{O}(1.70 \mathrm{~g}, 7.78 \mathrm{mmol}, 1.5$ equiv.) in dichloromethane $(52 \mathrm{~mL})$. After $4 \mathrm{~h}$, TLC showed full completion. $\mathrm{H}_{2} \mathrm{O}$ was added to the mixture, the layers were separated and the aqueous layer was extracted three times with DCM. The organic layer was dried over MgSO4, concentrated under vacuum and the crude product was purified by chromatography under silica gel to give the desired product tert-butyl $((1 \mathrm{H}-$ indol-4-yl)methyl)(naphthalen-2-ylmethyl)carbamate. (gradient from 0 to $20 \%$ Heptane:EtOAc) as an oil (1.38 g, $3.57 \mathrm{mmol}, 69 \%)$. The product appears as rotamers by ${ }^{1} \mathrm{H}$ NMR and ${ }^{13} \mathrm{C}$ NMR. IR (neat) $v_{\max }=3316,2976,2927,1666,1412,1365,1247,1161$, $1115,752 \mathrm{~cm}^{-1} .{ }^{1} \mathbf{H}$ NMR $\left(\mathrm{CDCl}_{3}, 500 \mathrm{MHz}\right) \delta=8.30$ (brs, 1H), 7.88$7.74(\mathrm{~m}, 3 \mathrm{H}), 7.61(\mathrm{~s}, 1 \mathrm{H}), 7.52-7.33(\mathrm{~m}, 4 \mathrm{H}), 7.21(\mathrm{t}, J=2.8 \mathrm{~Hz}, 1 \mathrm{H})$, $7.18(\mathrm{t}, J=7.7 \mathrm{~Hz}, 1 \mathrm{H}), 6.98(\mathrm{~d}, J=7.2 \mathrm{~Hz}, 1 \mathrm{H}), 6.72-6.50(\mathrm{~m}, 1 \mathrm{H})$, 4.86-4.67 (m, 2H), 4.63-4.43 (m, 2H), $1.55(\mathrm{~s}, 9 \mathrm{H}) .{ }^{13} \mathrm{C}\left\{{ }^{1} \mathrm{H}\right\}$ NMR $\left(\mathrm{CDCl}_{3}, 75 \mathrm{MHz}\right) \delta=136.2\left(\mathrm{C}_{\mathrm{q}}\right), 135.9\left(\mathrm{C}_{\mathrm{q}}\right), 133.5\left(\mathrm{C}_{\mathrm{q}}\right), 132.9\left(\mathrm{C}_{\mathrm{q}}\right)$, $129.7\left(\mathrm{C}_{\mathrm{q}}\right), 128.4(\mathrm{CH}), 127.9(\mathrm{CH}), 127.8(\mathrm{CH}), 126.8\left(\mathrm{C}_{\mathrm{q}}\right), 126.4$ and $126.2(\mathrm{CH}), 126.2(\mathrm{CH}), 126.1$ and $125.8(\mathrm{CH}), 125.8(\mathrm{CH}), 124.2$ $(\mathrm{CH}), 122.0(\mathrm{CH}), 120.1$ and $118.8(\mathrm{CH}) 119.9\left(\mathrm{C}_{q}\right), 110.7$ and 110.4 (CH), 101.6 and $100.9(\mathrm{CH}), 80.2\left(\mathrm{C}_{\mathrm{q}}\right), 49.0$ and $49.0\left(\mathrm{CH}_{2}\right), 47.6$ $\left(\mathrm{CH}_{2}\right), 28.7\left(\mathrm{CH}_{3}\right)$. HRMS (ESI): $\mathrm{m} / \mathrm{z}$ : calc for $\mathrm{C}_{21} \mathrm{H}_{19} \mathrm{~N}_{2} \mathrm{O}_{2}{ }^{+} 331.1447$ $[\mathrm{M}-\mathrm{tBu} \cdot+2 \mathrm{H}]^{+}$, found $331.1443, \mathrm{~m} / \mathrm{z}$ : calcd for $\mathrm{C}_{27} \mathrm{H}_{29} \mathrm{~N}_{3} \mathrm{Na} \mathrm{O}_{2}{ }^{+}$ $450.2157\left[\mathrm{M}+\mathrm{Na}+\mathrm{CH}_{3} \mathrm{CN}\right]^{+}$, found 450.2157 .

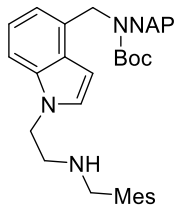

Acetonitrile $(11.9 \mathrm{~mL})$ was added on tert-butyl ((1H-indol-4-yl)methyl)(naphthalen-2-ylmethyl)carbamate ( $1.38 \mathrm{~g}, 3.57 \mathrm{mmol}, 1.0$ equiv.), 2chloroethan-1-amine hydrochloride $(476 \mathrm{mg}$, $4.11 \mathrm{mmol}, 1.15$ equiv.), sodium hydroxide (314 $\mathrm{mg}, 7.86 \mathrm{mmol}, 2.2$ equiv.) and tetrabutylammo- 
nium hydrogensulfate ( $49 \mathrm{mg}, 0.14 \mathrm{mmol}, 0.04$ equiv.). The mixture was stirred at reflux for $24 \mathrm{~h}$, then allowed to cool to rt, filtered over celite (wash with DCM), dried over $\mathrm{K}_{2} \mathrm{CO}_{3}$ and concentrated udder vacuum. This methodology routinely leads to 30 to $100 \%$ conversions. The crude product was dissolved in $\mathrm{MeOH}$ (18 $\mathrm{mL}$ ), and 2,4,6-trimethylbenzaldehyde (502 mg, $3.39 \mathrm{mmol}, 0.95$ equiv.) was added to the reaction mixture. After $16 \mathrm{~h}$ the reaction media was cooled to $0{ }^{\circ} \mathrm{C}$ before adding $\mathrm{NaBH}_{4}(148 \mathrm{mg}, 3.93$ mmol, 1.1 equiv.), then the mixture was allowed to reach room temperature. After 1 hour stirring, the volatiles were removed and the crude was next diluted in ethyl acetate and water. After the phases were separated, the aqueous phase was extracted twice by ethyl acetate then the combined organic phases were dried over $\mathrm{MgSO}_{4}$ and evaporated under vacuum. The crude mixture was then purified over column chromatography on silica gel to give the desired product tert-butyl (naphthalen-2-ylmethyl)((1-(2((2,4,6-trimethylbenzyl)amino)ethyl)- $1 \mathrm{H}$-indol-4-yl)methyl)carbamate. (gradient from 20 to $50 \%$ Heptane:EtOAc) as an oil (1.41 g, $2.51 \mathrm{mmol}, 15$ to $70 \%$ over 2 steps). The product appears as rotamers by ${ }^{1} \mathrm{H}$ NMR and ${ }^{13} \mathrm{C}$ NMR. IR (neat) $\mathbf{v}_{\max }=2973,2922,2859$, 1685, 1413, 1364, 1241, 1161, 1109, 748, $735 \mathrm{~cm}^{-1} .{ }^{1} \mathbf{H}$ NMR $\left(\mathrm{CDCl}_{3}, 500 \mathrm{MHz}\right) \delta=7.90-7.74(\mathrm{~m}, 3 \mathrm{H}), 7.61(\mathrm{~s}, 1 \mathrm{H}), 7.54-7.30(\mathrm{~m}$, $4 \mathrm{H}), 7.19(\mathrm{t}, J=7.8 \mathrm{~Hz}, 1 \mathrm{H}), 7.14(\mathrm{~d}, J=3 \mathrm{~Hz}, 1 \mathrm{H}), 6.98(\mathrm{~d}, J=7.2$ $\mathrm{Hz}, 1 \mathrm{H}), 6.82(\mathrm{~s}, 2 \mathrm{H}), 6.65-6.40(\mathrm{~m}, 1 \mathrm{H}), 4.91-4.66(\mathrm{~m}, 2 \mathrm{H}), 4.65-$ $4.43(\mathrm{~m}, 2 \mathrm{H}), 4.27(\mathrm{t}, J=6.2 \mathrm{~Hz}, 2 \mathrm{H}), 3.74(\mathrm{~s}, 2 \mathrm{H}), 3.13(\mathrm{t}, J=6.2 \mathrm{~Hz}$, 1H), $2.27(\mathrm{~s}, 6 \mathrm{H}), 2.24(\mathrm{~s}, 3 \mathrm{H}), 1.56(\mathrm{~s}, 9 \mathrm{H}) .{ }^{13} \mathrm{C}\left\{{ }^{1} \mathrm{H}\right\}$ NMR $\left(\mathrm{CDCl}_{3}, 75\right.$ $\mathrm{MHz}) \delta=137.0\left(\mathrm{C}_{\mathrm{q}}\right), 136.7\left(\mathrm{C}_{\mathrm{q}}\right), 136.4\left(\mathrm{C}_{\mathrm{q}}\right), 135.9\left(\mathrm{C}_{\mathrm{q}}\right), 133.5\left(\mathrm{C}_{\mathrm{q}}\right)$, $133.3\left(\mathrm{C}_{\mathrm{q}}\right), 132.8\left(\mathrm{C}_{\mathrm{q}}\right), 129.9\left(\mathrm{C}_{\mathrm{q}}\right), 129.1(2 \mathrm{CH}), 128.3(\mathrm{CH}), 128.1$ $(\mathrm{CH}), 127.8(\mathrm{CH}), 127.8(\mathrm{CH}), 126.8\left(\mathrm{C}_{\mathrm{q}}\right), 126.5$ and $126.2(\mathrm{CH})$, $126.2(\mathrm{CH}), 126.0$ and $125.8(\mathrm{CH}), 125.7(\mathrm{CH}), 121.5(\mathrm{CH}), 199.9$ $\left(\mathrm{C}_{\mathrm{q}}\right), 119.7$ and $118.4(\mathrm{CH}), 109.0$ and $108.8(\mathrm{CH}), 100.3$ and 99.6 $(\mathrm{CH}), 80.1\left(\mathrm{C}_{\mathrm{q}}\right), 49.5\left(\mathrm{CH}_{2}\right), 49.0\left(\mathrm{CH}_{2}\right), 47.6\left(\mathrm{CH}_{2}\right), 47.6\left(\mathrm{CH}_{2}\right), 46.9$ $\left(\mathrm{CH}_{2}\right), 28.7\left(3 \mathrm{CH}_{3}\right), 21.0\left(\mathrm{CH}_{3}\right), 19.6\left(\mathrm{CH}_{3}\right)$. HRMS (ESI): $\mathrm{m} / \mathrm{z}$ : calc for $\mathrm{C}_{37} \mathrm{H}_{44} \mathrm{~N}_{3} \mathrm{O}_{2}+562.3433[\mathrm{M}+\mathrm{H}]^{+}$, found 562.3376 .

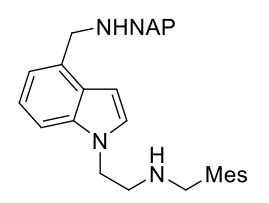

$\mathrm{HCl}(4 \mathrm{M}$ in dioxane, $5.4 \mathrm{~mL}, 21.5 \mathrm{mmol}$, 10 equiv.) was added on $\mathbf{S 8}(1.21 \mathrm{~g}, 2.15$ mmol, 1.0 equiv.). The reaction mixture was then stirred for $20 \mathrm{~h}$. The product precipitate was filtered, washed with 1,4-dioxane, and dried under vacuum. The crude product was dissolved in EtOAc then washed with a $1 \mathrm{M}$ aqueous $\mathrm{NaOH}$ solution. After the phases were separated, the aqueous phase was extracted twice by EtOAc then the combined organic phases were dried over $\mathrm{MgSO}_{4}$ and evaporated under vacuum. to give the desired product $5 \mathrm{~d}$. (as an oil $(650 \mathrm{mg}, 1.41 \mathrm{mmol}$, $65 \%) .{ }^{1} \mathrm{H} \mathrm{NMR}\left(\mathrm{CDCl}_{3}, 500 \mathrm{MHz}\right) \delta=7.85-7.79(\mathrm{~m}, 4 \mathrm{H}), 7.52(\mathrm{~d}, J$ $=9.0 \mathrm{~Hz}, 1 \mathrm{H}), 7.49-7.42(\mathrm{~m}, 2 \mathrm{H}), 7.30(\mathrm{~d}, J=8.1 \mathrm{~Hz}, 1 \mathrm{H}), 7.19(\mathrm{t}, J=$ $7.4 \mathrm{~Hz}, 2 \mathrm{H}), 7.16-7.10(\mathrm{~m}, 2 \mathrm{H}), 6.79(\mathrm{~s}, 1 \mathrm{H}), 6.54(\mathrm{~d}, J=3.2 \mathrm{~Hz}, 1 \mathrm{H})$, $4.26(\mathrm{t}, J=6.2 \mathrm{~Hz}, 2 \mathrm{H}), 4.14(\mathrm{~s}, 2 \mathrm{H}), 4.03(\mathrm{~s}, 2 \mathrm{H}), 3.72(\mathrm{~s}, 2 \mathrm{H}), 3.12$ $(\mathrm{t}, J=6.4 \mathrm{~Hz}, 2 \mathrm{H}), 2.25(\mathrm{~s}, 6 \mathrm{H}), 2.23(\mathrm{~s}, 3 \mathrm{H}), 1.84-1.34(\mathrm{bs}, 2 \mathrm{H})$. ${ }^{13} \mathrm{C}\left\{{ }^{1} \mathrm{H}\right\} \mathrm{NMR}\left(\mathrm{CDCl}_{3}, 75 \mathrm{MHz}\right) \delta=138.1\left(\mathrm{C}_{\mathrm{q}}\right), 137.0\left(\mathrm{C}_{\mathrm{q}}\right), 136.6\left(\mathrm{C}_{\mathrm{q}}\right)$, $136.3\left(C_{q}\right), 133.6\left(C_{q}\right), 133.3\left(C_{q}\right), 132.8\left(C_{q}\right), 132.5\left(C_{q}\right), 129.1(2$ $\mathrm{CH}), 128.1(\mathrm{CH}), 128.0(\mathrm{CH}), 127.9\left(\mathrm{C}_{\mathrm{q}}\right), 127.8(\mathrm{CH}), 127.8(\mathrm{CH})$, $126.9(\mathrm{CH}), 126.7(\mathrm{CH}), 126.0(\mathrm{CH}), 125.6(\mathrm{CH}), 121.7(\mathrm{CH}), 119.0$ (CH), $108.6(\mathrm{CH}), 99.5(\mathrm{CH}), 53.5\left(\mathrm{CH}_{2}\right), 51.2\left(\mathrm{CH}_{2}\right), 49.5\left(\mathrm{CH}_{2}\right), 47.6$ $\left(\mathrm{CH}_{2}\right), 46.9\left(\mathrm{CH}_{2}\right), 21.0\left(\mathrm{CH}_{3}\right), 19.6\left(\mathrm{CH}_{3}\right)$. HRMS (ESI): $\mathrm{m} / \mathrm{z}$ : calc for $\mathrm{C}_{32} \mathrm{H}_{36} \mathrm{~N}_{3}{ }^{+} 462.2909[\mathrm{M}+\mathrm{H}]^{+}$, found 462.2893 .

\section{Scope of the $\mathrm{C} 4 / \mathrm{N}$-Iso Pictet-Spengler reaction cascade with the} same aldehyde

General procedure 4: A mixture of tryptamine $5 \mathrm{~d}(0.17 \mathrm{mmol})$, Cat b (10 mol \%, $0.017 \mathrm{mmol}$ ) and $3 \AA$ molecular sieves ( $82 \mathrm{mg}$ for $0.17 \mathrm{mmol}$ of $\mathbf{5 d}$, powdered) in dichloromethane $(2.4 \mathrm{~mL}$ for 0.17 $\mathrm{mmol}$ of $\mathbf{5 d}$ ) was stirred for $5 \mathrm{~min}$ at room temperature under an argon atmosphere. Subsequently, aldehyde (3.0 equiv., 0.17 $\mathrm{mmol}$ ) was added and the mixture stirred at reflux for $24 \mathrm{~h}$. The mixture was filtered under celite and silica was added. After evaporation of the volatiles, the silica mixture was purified by chromatography under silica gel to give the desired product $\mathbf{9}$.

2-(naphthalen-2-ylmethyl)-1,11-diphenyl-10-(2,4,6-trimethylbenzyl)-2,3,8,9,10,11-hexahydro-1H-pyrazino[1',2':1,5]pyrrolo[4,3,2de] isoquinoline $9 a$

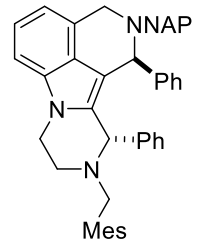

Anti 9a

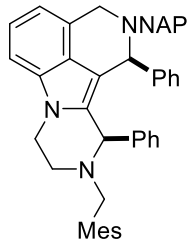

Syn $9 a$
Compound 9a was synthesized following the General procedure 4 using tryptamine $\mathbf{5 d}$ (100 mg, $0.22 \mathrm{mmol}, \quad 1.0$ equiv.), benzaldehyde (69 $\mathrm{mg}$, $0.65 \mathrm{mmol}, 3.0$ equiv.), Cat $\mathbf{b}$ (16.0 mg, $0.0217 \mathrm{mmol}, 10$ mol\%), M.S. $3 \AA ̊(104 \mathrm{mg})$, in DCM $(3.1 \mathrm{~mL})$ at $40^{\circ} \mathrm{C}$. Desired products 9 a were obtained after column chromatography on silica gel (gradient from 0 to $10 \%$ Heptane:EtOAc) as white amorphous solids (95 mg, $0.15 \mathrm{mmol}, 68$ $\%$, d.r 2:1 anti/syn). Anti 9a : IR (neat) $\mathbf{v}_{\max }=3055,3027,2960$, 2921, 2852, 1450, 1262, 1076, 1028, 1016, 711; $697 \mathrm{~cm}^{-1} .{ }^{1} \mathrm{H}$ NMR $\left(\mathrm{CDCl}_{3}, 500 \mathrm{MHz}\right) \delta=7.86-7.82(\mathrm{~m}, 1 \mathrm{H}), 7.80-7.74(\mathrm{~m}, 2 \mathrm{H}), 7.57(\mathrm{~s}$, $1 \mathrm{H})$, 7.51-7.41 (m, 3H), 7.25-7.21 (m, 3H), 7.21-7.11 (m, 4H), 7.01$6.91(\mathrm{~m}, 4 \mathrm{H}), 6.82-6.75(\mathrm{~m}, 4 \mathrm{H}), 4.30(\mathrm{~s}, 1 \mathrm{H}), 4.18(\mathrm{td}, J=3.0,11.5$ $\mathrm{Hz}, 1 \mathrm{H}), 4.06(\mathrm{dt}, J=4.0,10.8 \mathrm{~Hz}, 1 \mathrm{H}), 3.85(\mathrm{~d}, J=16.0 \mathrm{~Hz}, 1 \mathrm{H}), 3.70$ (s, 1H), $3.61(\mathrm{~d}, J=16.0 \mathrm{~Hz}, 1 \mathrm{H}), 3.56(\mathrm{~d}, J=14.0 \mathrm{~Hz}, 1 \mathrm{H}), 3.52(\mathrm{~d}, J$ $=12.7 \mathrm{~Hz}, 1 \mathrm{H}), 3.47(\mathrm{~d}, J=13.9 \mathrm{~Hz}, 1 \mathrm{H}), 3.21(\mathrm{~d}, J=12.6 \mathrm{~Hz}, 1 \mathrm{H})$, $3.12(\mathrm{td}, J=3.2,11.8 \mathrm{~Hz}, 1 \mathrm{H}), 2.74(\mathrm{dt}, J=43.5,11.3 \mathrm{~Hz}, 1 \mathrm{H}), 2.25$ $(\mathrm{s}, 3 \mathrm{H}), 2.19(\mathrm{~s}, 6 \mathrm{H}) .{ }^{13} \mathrm{C}\left\{{ }^{1} \mathrm{H}\right\}$ NMR $\left(\mathrm{CDCl}_{3}, 125 \mathrm{MHz}\right) \delta=140.9\left(\mathrm{C}_{\mathrm{q}}\right)$, $138.8\left(C_{q}\right), 137.5\left(C_{q}\right), 136.8\left(C_{q}\right), 135.6\left(C_{q}\right), 132.6\left(C_{q}\right), 132.0\left(C_{q}\right)$, $131.9\left(\mathrm{C}_{\mathrm{q}}\right), 131.8\left(\mathrm{C}_{\mathrm{q}}\right), 130.4\left(\mathrm{C}_{\mathrm{q}}\right), 129.1\left(\mathrm{C}_{\mathrm{q}}\right), 128.8(\mathrm{CH}), 128.1(\mathrm{CH})$, $127.5\left(\mathrm{C}_{\mathrm{q}}\right), 127.0(\mathrm{CH}), 126.9(\mathrm{CH}), 126.8(\mathrm{CH}), 126.7(\mathrm{CH}), 126.4$ $(\mathrm{CH}), 126.2(\mathrm{CH}), 125.8(\mathrm{CH}), 124.8(\mathrm{CH}), 124.4(\mathrm{CH}), 120.8(\mathrm{CH})$, 114.2 $(\mathrm{CH}), 106.5\left(\mathrm{C}_{\mathrm{q}}\right), 105.6(\mathrm{CH}), 67.3(\mathrm{CH}), 59.1(\mathrm{CH}), 57.8\left(\mathrm{CH}_{2}\right)$, $51.2\left(\mathrm{CH}_{2}\right), 46.4\left(\mathrm{CH}_{2}\right), 46.1\left(\mathrm{CH}_{2}\right), 42.0\left(\mathrm{CH}_{2}\right), 20.0\left(\mathrm{CH}_{3}\right), 19.6$ $\left(\mathrm{CH}_{3}\right)$. HRMS (ESI): $\mathrm{m} / \mathrm{z}$ : calc for $\mathrm{C}_{46} \mathrm{H}_{44} \mathrm{~N}_{3}{ }^{+} 638.3535[\mathrm{M}+\mathrm{H}]^{+}$, found 638.3552. Syn 9a : IR (neat) $\mathbf{v}_{\max }=3056,3027,2921,2853,1451$, $1291,1265,736,696 \mathrm{~cm}^{-1} .{ }^{1} \mathbf{H}$ NMR (CDCl, $\left.500 \mathrm{MHz}\right) \delta=7.91-7.81$ $(\mathrm{m}, 3 \mathrm{H}), 7.68(\mathrm{~s}, 1 \mathrm{H}), 7.59-7.47(\mathrm{~m}, 3 \mathrm{H}), 7.23-7.15(\mathrm{~m}, 2 \mathrm{H}), 6.98(\mathrm{t}$, $J=7.2 \mathrm{~Hz}, 1 \mathrm{H}), 6.94-6.88(\mathrm{~m}, 2 \mathrm{H}), 6.85-6.74(\mathrm{~m}, 5 \mathrm{H}), 6.62(\mathrm{t}, J=7.4$ $\mathrm{Hz}, 1 \mathrm{H}), 6.54(\mathrm{~d}, J=7.4 \mathrm{~Hz}, 1 \mathrm{H}), 4.56(\mathrm{~s}, 1 \mathrm{H}), 4.51(\mathrm{~s}, 1 \mathrm{H}), 4.19(\mathrm{td}$, $J=4.2,11.2 \mathrm{~Hz}, 1 \mathrm{H}$ ), 4.08 (ddd, J=4.5, 9.5, $11.3 \mathrm{~Hz}, 1 \mathrm{H}), 3.88$ (d, J $=16.3 \mathrm{~Hz}, 1 \mathrm{H}), 3.83-3.73(\mathrm{~m}, 1 \mathrm{H}), 3.71-3.61(\mathrm{~m}, 1 \mathrm{H}), 3.50(\mathrm{~d}, J=$ $130 \mathrm{~Hz}, 1 \mathrm{H}), 3.49$ (d, J=12.4 Hz, 1H), $3.27(\mathrm{~d}, J=12.2 \mathrm{~Hz}, 1 \mathrm{H}), 3.16$ (td, $J=4.3,12.0 \mathrm{~Hz}, 1 \mathrm{H}), 2.77$ (ddd, $J=3.5,9.4,12.1 \mathrm{~Hz}, 1 \mathrm{H}), 2.25$ $(\mathrm{s}, 3 \mathrm{H}), 2.20(\mathrm{~s}, 6 \mathrm{H}) .{ }^{13} \mathrm{C}\left\{{ }^{1} \mathrm{H}\right\}$ NMR $\left(\mathrm{CDCl}_{3}, 125 \mathrm{MHz}\right) \delta=140.1\left(\mathrm{C}_{\mathrm{q}}\right)$, $139.3\left(C_{q}\right), 138.5\left(C_{q}\right), 136.7\left(C_{q}\right), 133.6\left(C_{q}\right), 133.4\left(C_{q}\right), 133.0\left(C_{q}\right)$, $131.5\left(\mathrm{C}_{\mathrm{q}}\right), 131.5\left(\mathrm{C}_{\mathrm{q}}\right), 130.1\left(\mathrm{C}_{\mathrm{q}}\right), 129.5(\mathrm{CH}), 129.1(\mathrm{CH}), 128.8$ $(\mathrm{CH}), 128.0(\mathrm{CH}), 127.9(\mathrm{CH}), 127.8(\mathrm{CH}), 127.6(\mathrm{CH}), 127.5\left(\mathrm{C}_{\mathrm{q}}\right)$, $127.4(\mathrm{CH}), 127.2(\mathrm{CH}), 126.3(\mathrm{CH}), 126.0(\mathrm{CH}), 125.7\left(\mathrm{C}_{\mathrm{q}}\right), 125.7$ $(\mathrm{CH}), 121.9(\mathrm{CH}), 115.4(\mathrm{CH}), 106.8\left(\mathrm{C}_{\mathrm{q}}\right), 106.7(\mathrm{CH}), 66.7(\mathrm{CH}), 60.1$ $\left(\mathrm{CH}_{2}\right), 59.1(\mathrm{CH}), 52.0\left(\mathrm{CH}_{2}\right), 47.8\left(\mathrm{CH}_{2}\right), 46.5\left(\mathrm{CH}_{2}\right), 42.4\left(\mathrm{CH}_{2}\right), 21.0$ $\left(\mathrm{CH}_{3}\right), 20.5\left(\mathrm{CH}_{3}\right)$. HRMS (ESI): $\mathrm{m} / \mathrm{z}$ : calc for $\mathrm{C}_{46} \mathrm{H}_{44} \mathrm{~N}_{3}{ }^{+} 638.3535$ $[\mathrm{M}+\mathrm{H}]^{+}$, found 638.3568 .

2-(naphthalen-2-ylmethyl)-1,11-bis(3-(trifluoromethyl)phenyl)10-(2,4,6-trimethylbenzyl)-2,3,8,9,10,11-hexahydro-1 $\mathrm{H}$-pyrazino[1',2':1,5]pyrrolo[4,3,2-de]isoquinoline $9 \mathrm{~b}$ 


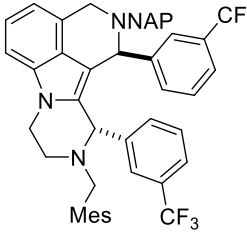

Anti 9b

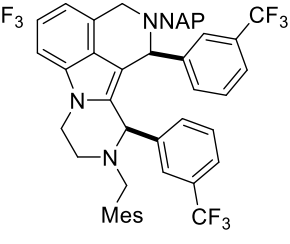

Syn 9b
Compound $9 \mathrm{~b}$ was synthesized following the General procedure 4 using tryptamine 5d $(100 \mathrm{mg}$, $0.22 \mathrm{mmol}, \quad 1.0$ equiv.), 3-(trifluoromethyl)benzalde-

hyde (113.15 mg, $0.65 \mathrm{mmol}, 3.0$ equiv.), Cat b (16.0 mg, 0.0217 mmol, $10 \mathrm{~mol} \%)$, M.S. $3 \AA$ (104 mg), in DCM $(3.1 \mathrm{~mL})$ at $40^{\circ} \mathrm{C}$. Desired products $9 \mathbf{b}$ were obtained after column chromatography on silica gel (gradient from 0 to $10 \%$ Heptane:EtOAc) as white amorphous solids ( $98 \mathrm{mg}, 0.12 \mathrm{mmol}, 58 \%$, d.r 1:4 anti/syn). Anti 9b : IR (neat) $\mathbf{v}_{\max }=3334,2970,2924,1327,1163,1120,1094,1072$, 746, $702 \mathrm{~cm}^{-1} .{ }^{1} \mathbf{H}$ NMR $\left(\mathrm{CDCl}_{3}, 500 \mathrm{MHz}\right) \delta=7.87-7.84(\mathrm{~m}, 1 \mathrm{H})$, $7.77(\mathrm{~d}, J=8.4 \mathrm{~Hz}, 2 \mathrm{H}), 7.55-7.46(\mathrm{~m}, 4 \mathrm{H}), 7.43-7.33(\mathrm{~m}, 4 \mathrm{H}), 7.25$ (s, $1 \mathrm{H}), 7.22-7.17(\mathrm{~m}, 2 \mathrm{H}), 7.09(\mathrm{~d}, J=7.6 \mathrm{~Hz}, 1 \mathrm{H}), 6.99-6.93(\mathrm{~m}$, $2 \mathrm{H}), 6.83-6.78(\mathrm{~m}, 2 \mathrm{H}), 4.33(\mathrm{~s}, 1 \mathrm{H}), 4.21(\mathrm{td}, J=2.8,11.3 \mathrm{~Hz}, 1 \mathrm{H})$, $4.08(\mathrm{dt}, J=4.1,11.2 \mathrm{~Hz}, 1 \mathrm{H}), 3.81(\mathrm{~d}, J=16.5 \mathrm{~Hz}, 1 \mathrm{H}), 3.66(\mathrm{~d}, J=$ $8.2 \mathrm{~Hz}, 1 \mathrm{H}), 3.63(\mathrm{~s}, 1 \mathrm{H}), 3.53(\mathrm{~d}, J=13.9 \mathrm{~Hz}, 1 \mathrm{H}), 3.48(\mathrm{~d}, J=14.0$ $\mathrm{Hz}, 1 \mathrm{H}$ ), 3.42 (d, $J=12.2 \mathrm{~Hz}, 1 \mathrm{H}$ ), 3.23 (d, $J=12.3 \mathrm{~Hz}, 1 \mathrm{H}$ ), 3.13 (td, $J=4.2,11.8 \mathrm{~Hz}, 1 \mathrm{H}), 2.76(\mathrm{dt}, J=3.8,11.5 \mathrm{~Hz}, 1 \mathrm{H}), 2.24(\mathrm{~s}, 3 \mathrm{H})$, $2.16(\mathrm{~s}, 6 \mathrm{H}) .{ }^{13} \mathrm{C}\left\{{ }^{1} \mathrm{H}\right\}$ NMR $\left(\mathrm{CDCl}_{3}, 75 \mathrm{MHz}\right) \delta=142.6\left(\mathrm{C}_{\mathrm{q}}\right), 141.0$ $\left(C_{q}\right), 138.4\left(C_{q}\right), 137.0\left(C_{q}\right), 137.0\left(C_{q}\right), 1335\left(C_{q}\right), 133.1\left(C_{q}\right), 133.0$ $\left(\mathrm{C}_{\mathrm{q}}\right), 132.2(\mathrm{CH}), 132.2(\mathrm{CH}), 131.8\left(\mathrm{C}_{\mathrm{q}}\right), 130.8\left(\mathrm{C}_{\mathrm{q}}\right), 130.7\left(\mathrm{C}_{\mathrm{q}}\right), 130.3$ $\left(\mathrm{C}_{\mathrm{q}}\right), 129.3(\mathrm{CH}), 128.8(\mathrm{CH}), 128.6(\mathrm{CH}), 128.3\left(\mathrm{C}_{\mathrm{q}}\right), 128.3(\mathrm{q}, \mathrm{C}-\mathrm{F}$, $\left.{ }^{1} J_{\mathrm{C}-\mathrm{F}}=252.5 \mathrm{~Hz}, \mathrm{Cq}\right), 128.1(\mathrm{CH}), 128.0\left(\mathrm{q}, \mathrm{C}-\mathrm{F},{ }^{1} J_{\mathrm{C}-\mathrm{F}}=248.3 \mathrm{~Hz}, \mathrm{Cq}\right)$, $127.9(\mathrm{CH}), 127.7(\mathrm{CH}), 127.1(\mathrm{CH}), 127.0(\mathrm{CH}), 126.5\left(\mathrm{~d},{ }^{3} J_{\mathrm{C}-\mathrm{F}}=3.3\right.$ $\mathrm{Hz}, \mathrm{CH}), 126.0(\mathrm{CH}), 125.7\left(\mathrm{~d}, \mathrm{C}-\mathrm{F},{ }^{3} \mathrm{~J}_{\mathrm{C}-\mathrm{F}}=3.9 \mathrm{~Hz}, \mathrm{CH}\right), 125.6(\mathrm{CH})$, $125.4\left(\mathrm{C}_{\mathrm{q}}\right), 125.0\left(\mathrm{~d}, \mathrm{C}-\mathrm{F},{ }^{3} \mathrm{~J}_{\mathrm{C}-\mathrm{F}}=3.3 \mathrm{~Hz}, \mathrm{CH}\right), 123.9\left(\mathrm{~d}, \mathrm{C}-\mathrm{F},{ }^{3} \mathrm{~J}_{\mathrm{C}-\mathrm{F}}=3.2\right.$ $\mathrm{Hz}, \mathrm{CH}), 122.3(\mathrm{CH}), 115.5(\mathrm{CH}), 106.9(\mathrm{CH}), 106.8\left(\mathrm{C}_{\mathrm{q}}\right), 67.8(\mathrm{CH})$, $59.3(\mathrm{CH}), 58.7\left(\mathrm{CH}_{2}\right), 52.4\left(\mathrm{CH}_{2}\right), 47.6\left(\mathrm{CH}_{2}\right), 47.1\left(\mathrm{CH}_{2}\right), 42.9\left(\mathrm{CH}_{2}\right)$, $21.0\left(\mathrm{CH}_{3}\right), 20.5\left(\mathrm{CH}_{3}\right) .{ }^{19 F} \mathrm{NMR}\left(\mathrm{CDCl}_{3}, 282 \mathrm{MHz}\right) \delta=-62.4,-62.8$. HRMS (ESI): $m / z$ : calc for $\mathrm{C}_{48} \mathrm{H}_{42} \mathrm{~F}_{6} \mathrm{~N}_{3}{ }^{+} 774.3283[\mathrm{M}+\mathrm{H}]^{+}$, found 774.3231. Syn 9b : IR (neat) $\mathbf{v}_{\max }=3340,2973,2924,1327,1163$, $1119,1092,1071,1047,736,700 \mathrm{~cm}^{-1} .{ }^{1} \mathrm{H}$ NMR $\left(\mathrm{CDCl}_{3}, 500 \mathrm{MHz}\right)$ $\delta=7.93-7.83(\mathrm{~m}, 3 \mathrm{H}), 7.69(\mathrm{~s}, 1 \mathrm{H}), 7.58-7.50(\mathrm{~m}, 3 \mathrm{H}), 7.28-7.19(\mathrm{~m}$, $3 \mathrm{H}), 7.14(\mathrm{~s}, 3 \mathrm{H}), 7.08(\mathrm{~d}, J=7.7 \mathrm{~Hz}, 1 \mathrm{H}), 7.05-6.98(\mathrm{~m}, 2 \mathrm{H}), 6.87-$ $6.82(\mathrm{~m}, \mathrm{H}), 6.79(\mathrm{t}, J=7.7 \mathrm{~Hz}, 1 \mathrm{H}), 6.69(\mathrm{~d}, J=7.7 \mathrm{~Hz}, 1 \mathrm{H}), 4.65(\mathrm{~s}$, $1 \mathrm{H}), 4.60(\mathrm{~s}, 1 \mathrm{H}), 4.23(\mathrm{td}, J=4.3,11.2 \mathrm{~Hz}, 1 \mathrm{H}), 4.15(\mathrm{dt}, J=3.2$, $10.0 \mathrm{~Hz}, 1 \mathrm{H}), 3.80(\mathrm{~d}, J=16.1 \mathrm{~Hz}, 1 \mathrm{H}), 3.76(\mathrm{~d}, J=13.0 \mathrm{~Hz}, 1 \mathrm{H}), 3.67$ (d, $J=14.1 \mathrm{~Hz}, 1 \mathrm{H}), 3.53(\mathrm{~d}, J=13.2 \mathrm{~Hz}, 1 \mathrm{H}), 3.43(\mathrm{~d}, J=12.5 \mathrm{~Hz}$, $1 \mathrm{H}), 3.32(\mathrm{~d}, J=12.4 \mathrm{~Hz}, 1 \mathrm{H}), 3.17(\mathrm{td}, J=4.0,12.1 \mathrm{~Hz}, 1 \mathrm{H}), 2.82$ (ddd, $J=3.7 ; 10.2 ; 12.6 \mathrm{~Hz}, 1 \mathrm{H}$ ), $2.28(\mathrm{~s}, 3 \mathrm{H}), 2.20(\mathrm{~s}, 6 \mathrm{H}) .{ }^{13} \mathrm{C}\left\{{ }^{1} \mathrm{H}\right\}$ NMR $\left(\mathrm{CDCl}_{3}, 75 \mathrm{MHz}\right) \delta=141.3\left(\mathrm{C}_{\mathrm{q}}\right), 141.0\left(\mathrm{C}_{\mathrm{q}}\right), 138.4\left(\mathrm{C}_{\mathrm{q}}\right), 137.0$ $\left(C_{q}\right), 136.9\left(C_{q}\right), 1336\left(C_{q}\right), 133.3\left(C_{q}\right), 133.1\left(C_{q}\right), 132.7(C H), 131.6$ $(\mathrm{CH}), 130.8\left(\mathrm{C}_{\mathrm{q}}\right), 130.5\left(\mathrm{C}_{\mathrm{q}}\right), 129.8\left(\mathrm{C}_{\mathrm{q}}\right), 129.4\left(\mathrm{C}_{\mathrm{q}}\right), 129.3(2 \mathrm{CH})$, $128.4\left(\mathrm{C}_{\mathrm{q}}\right), 128.3\left(\mathrm{q}, \mathrm{C}-\mathrm{F},{ }^{1} \mathrm{~J}_{\mathrm{C}-\mathrm{F}}=260.0 \mathrm{~Hz}, \mathrm{Cq}\right), 128.3(\mathrm{CH}), 128.2$ (CH), $127.9(2 \mathrm{CH}), 127.8(\mathrm{CH}), 127.8(\mathrm{CH}), 127.5(\mathrm{CH}), 127.4(\mathrm{q}, \mathrm{C}-$ $\left.\mathrm{F},{ }^{1} J_{\mathrm{C}-\mathrm{F}}=276.5 \mathrm{~Hz}, \mathrm{Cq}\right), 126.6(\mathrm{CH}), 126.1\left(\mathrm{q}, \mathrm{C}-\mathrm{F},{ }^{3} J_{\mathrm{C}-\mathrm{F}}=3.3 \mathrm{~Hz}, \mathrm{CH}\right)$, $125.8(\mathrm{CH}), 125.6\left(\mathrm{C}_{\mathrm{q}}\right), 125.2\left(\mathrm{q}, \mathrm{C}-\mathrm{F},{ }^{3} \mathrm{~J}_{\mathrm{C}-\mathrm{F}}=3.8 \mathrm{~Hz}, \mathrm{CH}\right), 124.5$ (q, C$\left.\mathrm{F}, 3 \mathrm{~J}_{\mathrm{C}-\mathrm{F}}=3.3 \mathrm{~Hz}, \mathrm{CH}\right), 123.6\left(\mathrm{q}, \mathrm{C}-\mathrm{F},{ }^{3} \mathrm{~J}_{\mathrm{C}-\mathrm{F}}=3.3 \mathrm{~Hz}, \mathrm{CH}\right), 122.4(\mathrm{CH})$, $115.7(\mathrm{CH}), 107.0(\mathrm{CH}), 106.4\left(\mathrm{C}_{\mathrm{q}}\right), 66.6(\mathrm{CH}), 59.6(\mathrm{CH}), 59.1\left(\mathrm{CH}_{2}\right)$, $52.3\left(\mathrm{CH}_{2}\right), 47.7\left(\mathrm{CH}_{2}\right), 46.6\left(\mathrm{CH}_{2}\right), 42.5\left(\mathrm{CH}_{2}\right), 21.0\left(\mathrm{CH}_{3}\right), 20.5$ $\left(\mathrm{CH}_{3}\right) .{ }^{19 F} \mathrm{NMR}\left(\mathrm{CDCl}_{3}, 282 \mathrm{MHz}\right) \delta=-62.5,-62.9$. HRMS (ESI): $\mathrm{m} / \mathrm{z}$ : calc for $\mathrm{C}_{48} \mathrm{H}_{42} \mathrm{~F}_{6} \mathrm{~N}_{3}+774.3283[\mathrm{M}+\mathrm{H}]^{+}$, found 774.3218 . 2-(4-(naphthalen-2-ylmethyl)-3-(quinolin-4-yl)-4,5-dihydropyrrolo[4,3,2-de] isoquinolin-1(3H)-yl)- N-(2,4,6-trimethylbenzyl)ethan-1-amine $9 c$

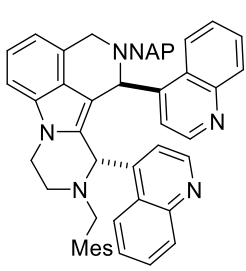

Compound 9c was synthesized following the General procedure 4 using tryptamine 5d (75 mg, $0.16 \mathrm{mmol}, 1.0$ equiv.), quinoline-4-carbaldehyde (77 mg, $0.49 \mathrm{mmol}, 3.0$ equiv.), Cat b (12.0 mg, $0.0162 \mathrm{mmol}, 10$ mol\%), M.S. $3 \AA$ ( $78 \mathrm{mg})$, in DCM $(2.3 \mathrm{~mL})$ at $40^{\circ} \mathrm{C}$. The desired product $9 \mathrm{c}$ was obtained after column chromatography on silica gel (gradient from 40 to $60 \%$ Heptane:EtOAc) as a single diastereoisomer (84 mg, $0.14 \mathrm{mmol}, 88 \%$ ). IR (neat) $\boldsymbol{v}_{\max }=3055,2926,1688$, $1508,1455,1365,1247,1164,756 \mathrm{~cm}^{-1} .{ }^{1} \mathrm{H} \mathrm{NMR}\left(\mathrm{CDCl}_{3}, 500 \mathrm{MHz}\right)$ $\delta=8.70(\mathrm{~s}, 1 \mathrm{H}), 8.49(\mathrm{bs}, 1 \mathrm{H}), 8.13(\mathrm{~d}, J=8.6 \mathrm{~Hz}, 1 \mathrm{H}), 8.08(\mathrm{bs}, 1 \mathrm{H})$, 7.84-7.79 (m, 1H), 7.74-7.64 (m, 3H), 7.56-7.42 (m, 3H), 7.40-7.21 $(\mathrm{m}, 6 \mathrm{H}), 7.21-6.97(\mathrm{~m}, 3 \mathrm{H}), 6.80(\mathrm{~s}, 2 \mathrm{H}), 6.77(\mathrm{~d}, J=7.3 \mathrm{~Hz}, 1 \mathrm{H})$, $6.63(\mathrm{~d}, J=4.4 \mathrm{~Hz}, 1 \mathrm{H}), 4.94(\mathrm{~s}, 1 \mathrm{H}), 4.46-4.32(\mathrm{~m}, 2 \mathrm{H}), 3.98(\mathrm{~s}, 1 \mathrm{H})$, 3.64-3.49 (m, 2H), $3.42(\mathrm{~d}, J=16.7 \mathrm{~Hz}, 1 \mathrm{H}), 3.38-3.31(2 \mathrm{H}), 3.31-$ $3.20(\mathrm{~m}, 1 \mathrm{H}), 3.02-2.73(\mathrm{~m}, 2 \mathrm{H}), 2.27(\mathrm{~s}, 3 \mathrm{H}), 2.11(\mathrm{~s}, 6 \mathrm{H}) .{ }^{13} \mathrm{C}\left\{{ }^{1} \mathrm{H}\right\}$ NMR $\left(\mathrm{CDCl}_{3}, 75 \mathrm{MHz}\right) \delta=149.8(\mathrm{CH}), 149.7(\mathrm{CH}), 148.8\left(\mathrm{C}_{\mathrm{q}}\right), 148.6$ $\left(C_{q}\right), 147.2\left(C_{q}\right), 144.9\left(C_{q}\right), 138.4\left(C_{q}\right), 137.1\left(C_{q}\right), 136.9\left(C_{q}\right), 133.3$ $\left(C_{q}\right), 133.1\left(C_{q}\right), 132.8\left(C_{q}\right), 131.1\left(C_{q}\right), 130.2\left(C_{q}\right), 130.1(C H), 129.7$ $(\mathrm{CH}), 129.4(\mathrm{CH}), 129.2(\mathrm{CH}), 129.1(\mathrm{CH}), 128.8\left(\mathrm{C}_{\mathrm{q}}\right), 127.9(\mathrm{CH})$, 127,8 (CH), $127.7(\mathrm{CH}), 127.5\left(\mathrm{C}_{\mathrm{q}}\right), 127.3(\mathrm{CH}), 127.1(\mathrm{CH}), 127.0$ $\left(\mathrm{C}_{\mathrm{q}}\right), 126.8\left(\mathrm{C}_{\mathrm{q}}\right), 126.6(\mathrm{CH}), 125.9(\mathrm{CH}), 125.9(\mathrm{CH}), 125.6(\mathrm{CH})$, $124.4(\mathrm{CH}), 123.0(\mathrm{CH}), 122.6(\mathrm{CH}), 120.9(\mathrm{CH}), 116.2(\mathrm{CH}), 107.1$ $(\mathrm{CH}), 104.7\left(\mathrm{C}_{\mathrm{q}}\right), 59.0\left(\mathrm{CH}_{2}\right), 57.9(\mathrm{CH}), 53.0\left(\mathrm{CH}_{2}\right), 47.9\left(\mathrm{CH}_{2}\right), 45.4$ $\left(\mathrm{CH}_{2}\right), 43.1\left(\mathrm{CH}_{2}\right), 21.0\left(\mathrm{CH}_{3}\right), 20.6\left(\mathrm{CH}_{3}\right)$. Note that one of the benzylic $\mathrm{CH}$ does not resonate well in the $13 \mathrm{C}$ NMR spectrum, it is estimated around 67 ppm. HRMS (ESI): $\mathrm{m} / \mathrm{z}$ : calc for $\mathrm{C}_{52} \mathrm{H}_{46} \mathrm{~N}_{5}{ }^{+}$ $740.3753[\mathrm{M}+\mathrm{H}]^{+}$, found 740.3784 .

2-(3-(4-bromophenyl)-4-(naphthalen-2-ylmethyl)-4,5-dihydropyrrolo[4,3,2-de]isoquinolin-1(3H)-yl)-N-(2,4,6-trimethylbenzyl)ethan-1-amine 10a

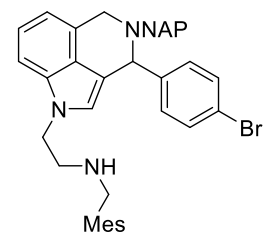

A mixture of tryptamine $\mathbf{5 d}(84 \mathrm{mg}, 0.18$ mmol, 1.0 equiv.), Cat b (13.4 mg, 0.0182 $\mathrm{mmol}, 10 \mathrm{~mol} \%)$ and M.S. $3 \AA \AA$ ( $87 \mathrm{mg})$, in DCM $(2.6 \mathrm{~mL})$ was stirred for $5 \mathrm{~min}$ at room temperature under an argon atmosphere. Subsequently, 4-bromobenzaldehyde ( $34 \mathrm{mg}, 0.18 \mathrm{mmol}, 1.0$ equiv.), was added and the mixture stirred at reflux for $24 \mathrm{~h}$. The mixture was filtered under celite and silica was added. After evaporation of the volatiles, the silica residue was purified by chromatography under silica gel gradient from 0 to $50 \%$ Heptane:EtOAc) to give product $10 \mathrm{a}$ as an oil ( $81 \mathrm{mg}, 0.13 \mathrm{mmol}, 71 \%$ ). IR (neat) $\boldsymbol{v}_{\max }=3315,3051$, 2922, 2852, 1459, 1263, 1010, $734 \mathrm{~cm}^{-1} .{ }^{1} \mathrm{H}$ NMR ( $\mathrm{CDCl}_{3}, 300 \mathrm{MHz}$ ) $\delta=7.89-7.76(\mathrm{~m}, 3 \mathrm{H}), 7.71(\mathrm{~s}, 1 \mathrm{H}), 7.58(\mathrm{~d}, J=9.0 \mathrm{~Hz}, 1 \mathrm{H}), 7.52-$ $7.45(\mathrm{~m}, 2 \mathrm{H}), 7,44-7.37(\mathrm{~m}, 2 \mathrm{H}), 7.26-7.14(\mathrm{~m}, 4 \mathrm{H}), 6.83(\mathrm{~s}, 2 \mathrm{H})$, $6.79(\mathrm{~d}, J=6.6 \mathrm{~Hz}, 1 \mathrm{H}), 6.71(\mathrm{~s}, 1 \mathrm{H}), 4.98(\mathrm{~s}, 1 \mathrm{H}), 4.27(\mathrm{t}, J=6.1 \mathrm{~Hz}$, $2 \mathrm{H}), 4.05(\mathrm{~d}, J=15.8 \mathrm{~Hz}, 1 \mathrm{H}), 3.86(\mathrm{~d}, J=13.4 \mathrm{~Hz}, 1 \mathrm{H}), 3.79(\mathrm{~d}, J=$ $16.2 \mathrm{~Hz}, 1 \mathrm{H}), 3.74(\mathrm{~s}, 2 \mathrm{H}), 3.69(\mathrm{~d}, J=13.6 \mathrm{~Hz}, 1 \mathrm{H}), 3.16(\mathrm{t}, J=5.8$ $\mathrm{Hz}, 1 \mathrm{H}), 2.30-2.20(\mathrm{~m}, 9 \mathrm{H}) .{ }^{13} \mathrm{C}\left\{{ }^{1} \mathrm{H}\right\} \mathrm{NMR}\left(\mathrm{CDCl}_{3}, 75 \mathrm{MHz}\right) \delta=142.0$ $\left(C_{q}\right), 137.4\left(C_{q}\right), 136.9\left(C_{q}\right), 136.7\left(C_{q}\right), 134.1\left(C_{q}\right), 133.5\left(C_{q}\right), 133.4$ $\left(C_{q}\right), 133.0\left(C_{q}\right), 131.2(\mathrm{CH}), 130.5(\mathrm{CH}), 129.2(\mathrm{CH}), 129.0\left(\mathrm{C}_{\mathrm{q}}\right)$, $128.1(\mathrm{CH}), 127.8(\mathrm{CH}), 127.8(\mathrm{CH}), 127.4(\mathrm{CH}), 127.4(\mathrm{CH}), 126.0$ $(\mathrm{CH}), 125.7\left(\mathrm{C}_{\mathrm{q}}\right), 125.7(\mathrm{CH}), 123.3(\mathrm{CH}), 122.8(\mathrm{CH}), 121.0\left(\mathrm{C}_{\mathrm{q}}\right)$, 114.7 (CH), $112.5\left(\mathrm{C}_{\mathrm{q}}\right), 107.6(\mathrm{CH}), 61.0\left(\mathrm{CH}_{2}\right), 58.9(\mathrm{CH}), 49.9\left(\mathrm{CH}_{2}\right)$, $49.6\left(\mathrm{CH}_{2}\right), 47.7\left(\mathrm{CH}_{2}\right), 47.1\left(\mathrm{CH}_{2}\right), 21.0\left(\mathrm{CH}_{3}\right), 19.6\left(\mathrm{CH}_{3}\right)$ HRMS (ESI): $\mathrm{m} / \mathrm{z}$ : calc for $\mathrm{C}_{39} \mathrm{H}_{39}{ }^{79} \mathrm{BrN}_{3}{ }^{+} 628.2327[\mathrm{M}+\mathrm{H}]^{+}$, found 628.2301, calc for $\mathrm{C}_{39} \mathrm{H}_{39}{ }^{81} \mathrm{BrN}_{3}{ }^{+} 630.2327[\mathrm{M}+\mathrm{H}]^{+}$, found 630.2307 . 
Scope of the C4/N-Iso Pictet-Spengler reaction cascade with two different aldehydes

General procedure 5: A mixture of tryptamine $5 \mathbf{d}(0.17$ mmol), Cat b (10 mol \%, $0.017 \mathrm{mmol}$,$) and 3 \AA$ molecular sieves ( $82 \mathrm{mg}$ for $0.17 \mathrm{mmol}$ of $\mathbf{5 d}$, powdered) in dichloromethane (2.4 $\mathrm{mL}$ for $0.17 \mathrm{mmol}$ of $\mathbf{5 d}$ ) was stirred for $5 \mathrm{~min}$ at room temperature under an argon atmosphere. Subsequently, aldehyde a (1.0 equiv., $0.17 \mathrm{mmol}$ ) was added and the mixture stirred for $15 \mathrm{~h}$. Then aldehyde $\mathbf{b}$ ( 2.0 equiv., $0.35 \mathrm{mmol}$ ) was added and the mixture stirred at reflux for $24 \mathrm{~h}$. The mixture was filtered under celite and silica was added. After evaporation of the volatiles, the silica mixture was purified by chromatography under silica gel to give the desired product 11.

1-(4-bromophenyl)-2-(naphthalen-2-ylmethyl)-11-phenyl-10(2,4,6-trimethylbenzyl)-2,3,8,9,10,11-hexahydro- $1 \mathrm{H}$-pyrazino[1',2':1,5]pyrrolo[4,3,2-de] isoquinoline 11a

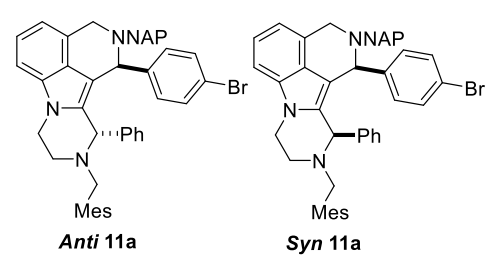

Sequential procedure: $A$ mixture of tryptamine 10a $(65 \mathrm{mg}, 0.14 \mathrm{mmol}$, 1.0 equiv.), Cat $\mathbf{b}(10.4$ $\mathrm{mg}, 0.014 \mathrm{mmol}, 10 \mathrm{~mol}$ $\%)$ and $3 \AA$ molecular sieves $(68 \mathrm{mg})$ in dichloromethane $(2.0 \mathrm{~mL})$

was stirred for $5 \mathrm{~min}$ at room temperature under an argon atmosphere. Subsequently, benzaldehyde $(30 \mathrm{mg}, 0.28 \mathrm{mmol}, 2.0$ equiv.) was added and the mixture stirred at $40^{\circ} \mathrm{C}$ for $24 \mathrm{~h}$. The mixture was filtered under celite and silica was added. The desired products 11a were obtained after column chromatography on silica gel (gradient from 0 to $10 \%$ Heptane:EtOAc) as an oil $(70 \mathrm{mg}$, $0.10 \mathrm{mmol}, 70 \%$, d.r 1:3 anti/syn). One-pot procedure: Compound 11a was synthesized following the General procedure 5 using tryptamine $\mathbf{5 d}$ ( $31 \mathrm{mg}, 0.07 \mathrm{mmol}, 1.0$ equiv.), 4-bromobenzaldehyde (12 mg, $0.07 \mathrm{mmol}, 1.0$ equiv.), Cat b $(5.0 \mathrm{mg}, 0.0067$ mmol, $10 \mathrm{~mol} \%)$, then benzaldehyde (14 mg, $0.13 \mathrm{mmol}, 2.0$ equiv.), M.S. $3 \AA$ ( $32 \mathrm{mg})$, in DCM $(1.0 \mathrm{~mL})$. The desired products 11a were obtained after column chromatography on silica gel (gradient from 0 to $10 \%$ Heptane:EtOAc) as an oil (29 mg, 0.05 mmol, $72 \%$, d.r 1:2 anti/syn). Anti 11a: IR (neat) $\mathbf{v}_{\max }=3054$, 2922, 2852, 1484, 1451, 1289, 1263, 1010, 736, $698 \mathrm{~cm}^{-1} .{ }^{1} \mathbf{H}$ NMR $\left(\mathrm{CDCl}_{3}, \mathbf{5 0 0 ~ M H z}\right) \delta=7.86-7.82(\mathrm{~m}, 1 \mathrm{H}), 7.79-7.74(\mathrm{~m}, 2 \mathrm{H}), 7.53(\mathrm{~s}$, $1 \mathrm{H}), 7.50-7.44(\mathrm{~m}, 2 \mathrm{H}), 7.41(\mathrm{~d}, J=8.6 \mathrm{~Hz}, 1 \mathrm{H}), 7.36(\mathrm{~d}, J=7.8 \mathrm{~Hz}$, $2 \mathrm{H}), 7.21-7.12(\mathrm{~m}, 4 \mathrm{H}), 6.96(\mathrm{t}, J=7.5 \mathrm{~Hz}, 2 \mathrm{H}), 6.83-6.78(\mathrm{~m}, 5 \mathrm{H})$, $6.76(\mathrm{~d}, J=6.7 \mathrm{~Hz}, 1 \mathrm{H}), 4.27(\mathrm{~s}, 1 \mathrm{H}), 4.18(\mathrm{td}, J=3.0,11.1 \mathrm{~Hz}, 1 \mathrm{H})$, $4.06(\mathrm{dt}, J=4.2,11.2 \mathrm{~Hz}, 1 \mathrm{H}), 3.78(\mathrm{~d}, J=16.2 \mathrm{~Hz}, 1 \mathrm{H}), 3.59(\mathrm{~d}, J=$ $15.9 \mathrm{~Hz}, 1 \mathrm{H}), 3.59(\mathrm{~s}, 1 \mathrm{H}), 3.55-3.50(\mathrm{~m}, 2 \mathrm{H}), 3.44(\mathrm{~d}, J=13.8 \mathrm{~Hz}$, $1 \mathrm{H}), 3.20(\mathrm{~d}, J=12.5 \mathrm{~Hz}, 1 \mathrm{H}), 3.12(\mathrm{td}, J=2.9,11.6 \mathrm{~Hz}, 1 \mathrm{H}), 2.73$ (dt, $J=3.6,11.3 \mathrm{~Hz}, 1 \mathrm{H}), 2.25(\mathrm{~s}, 3 \mathrm{H}), 2.19(\mathrm{~s}, 6 \mathrm{H}) .{ }^{13} \mathrm{C}\left\{{ }^{1} \mathrm{H}\right\}$ NMR $\left(\mathrm{CDCl}_{3}, 125 \mathrm{MHz}\right) \delta=141.2\left(\mathrm{C}_{\mathrm{q}}\right), 139.7\left(\mathrm{C}_{\mathrm{q}}\right), 138.5\left(\mathrm{C}_{\mathrm{q}}\right), 137.5\left(\mathrm{C}_{\mathrm{q}}\right)$, $136.7\left(C_{q}\right), 133.6\left(C_{q}\right), 133.0\left(C_{q}\right), 133.0\left(C_{q}\right), 131.3\left(C_{q}\right), 131.0(2$ $\mathrm{CH}), 130.9(\mathrm{CH}), 129.8(\mathrm{CH}), 129.3\left(\mathrm{C}_{\mathrm{q}}\right), 129.2(\mathrm{CH}), 128.2\left(\mathrm{C}_{\mathrm{q}}\right)$, $128.0(\mathrm{CH}), 127.9(\mathrm{CH}), 127.7(\mathrm{CH}), 127.2(\mathrm{CH}), 127.2(\mathrm{CH}), 127.1$ $\left(\mathrm{C}_{\mathrm{q}}\right), 125.9(\mathrm{CH}), 125.5(\mathrm{CH}), 121.9(\mathrm{CH}), 120.7\left(\mathrm{C}_{\mathrm{q}}\right), 115.3(\mathrm{CH})$, $106.8\left(\mathrm{C}_{\mathrm{q}}\right), 106.7(\mathrm{CH}), 68.4(\mathrm{CH}), 59.5(\mathrm{CH}), 58.8\left(\mathrm{CH}_{2}\right), 52.3\left(\mathrm{CH}_{2}\right)$, $47.3\left(\mathrm{CH}_{2}\right), 43.1\left(2 \mathrm{CH}_{2}\right), 21.0\left(\mathrm{CH}_{3}\right), 20.6\left(\mathrm{CH}_{3}\right)$. HRMS (ESI): $\mathrm{m} / \mathrm{z}$ : calc. for $\mathrm{C}_{46} \mathrm{H}_{43}{ }^{79} \mathrm{BrN}_{3}{ }^{+} 716.2640[\mathrm{M}+\mathrm{H}]^{+}$, found 716.2622 , calc. for $\mathrm{C}_{39} \mathrm{H}_{39}{ }^{81} \mathrm{BrN}_{3}{ }^{+} 718.2620[\mathrm{M}+\mathrm{H}]^{+}$, found 718.2652. Syn 11a: IR (neat) $v_{\max }=3054,2958,2924,2871,1485,1451,1364,1290$, 1010, 750, $698 \mathrm{~cm}^{-1} .{ }^{1} \mathrm{H}$ NMR $\left(\mathrm{CDCl}_{3}, 500 \mathrm{MHz}\right) \delta=7.88-7.82(\mathrm{~m}$, $3 \mathrm{H}), 7.66(\mathrm{~s}, 1 \mathrm{H}), 7.53-7.46(\mathrm{~m}, 3 \mathrm{H}), 7.22-7.14(\mathrm{~m}, 2 \mathrm{H}), 7.00(\mathrm{~d}, J=$ $8.3 \mathrm{~Hz}, 2 \mathrm{H}), 6.86-6.77(\mathrm{~m}, 6 \mathrm{H}), 6.68(\mathrm{t}, J=7.5,2 \mathrm{H}), 6.40(\mathrm{~d}, J=8.5$ $\mathrm{Hz}, 2 \mathrm{H}), 4.55(\mathrm{~s}, 1 \mathrm{H}), 4.46(\mathrm{~s}, 1 \mathrm{H}), 4.21-4.15(\mathrm{~m}, 1 \mathrm{H}), 4.08(\mathrm{dt}, J=$
4.2, $10.4 \mathrm{~Hz}, 1 \mathrm{H}), 3.81$ (d, $J=16.3 \mathrm{~Hz}, 1 \mathrm{H}), 3.73(\mathrm{~d}, J=13.0 \mathrm{~Hz}, 1 \mathrm{H})$, $3.65(\mathrm{~d}, J=16.1 \mathrm{~Hz}, 1 \mathrm{H}), 3.48(\mathrm{~d}, J=13.0 \mathrm{~Hz}, 1 \mathrm{H}), 3.48(\mathrm{~d}, J=12.6$ $\mathrm{Hz}, 1 \mathrm{H}$ ), 3.27 (d, $J=12.4 \mathrm{~Hz}, 2 \mathrm{H}$ ), 3.20-3.11 (m, 1H), 2.76 (ddd, $J=$ 3.5, 9.4, $12.0 \mathrm{~Hz}, 1 \mathrm{H}), 2.25(\mathrm{~s}, 3 \mathrm{H}), 2.19(\mathrm{~s}, 6 \mathrm{H}) .{ }^{13} \mathrm{C}\left\{{ }^{1} \mathrm{H}\right\}$ NMR $\left(\mathrm{CDCl}_{3}\right.$, $125 \mathrm{MHz}) \delta=139.5\left(\mathrm{C}_{\mathrm{q}}\right), 139.5\left(\mathrm{C}_{\mathrm{q}}\right), 138.5\left(\mathrm{C}_{\mathrm{q}}\right), 137.3\left(\mathrm{C}_{\mathrm{q}}\right) 136.7$ $\left(C_{q}\right), 133.6\left(C_{q}\right), 133.4\left(C_{q}\right), 133.0\left(C_{q}\right), 131.7\left(C_{q}\right), 131.4\left(C_{q}\right), 130.5$ $(\mathrm{CH}), 130.3(\mathrm{CH}), 129.6(\mathrm{CH}), 129.1(\mathrm{CH}), 128.4\left(\mathrm{C}_{\mathrm{q}}\right), 128.1(\mathrm{CH})$, $127.8(\mathrm{CH}), 127.8(\mathrm{CH}), 127.7(\mathrm{CH}), 127.7(\mathrm{CH}), 127.6(\mathrm{CH}), 126.1$ $(\mathrm{CH}), 125.7(\mathrm{CH}), 125.7\left(\mathrm{C}_{\mathrm{q}}\right), 122.0\left(\mathrm{C}_{\mathrm{q}}\right), 122.0(\mathrm{CH}), 120.2\left(\mathrm{C}_{\mathrm{q}}\right)$, $115.4(\mathrm{CH}), 106.8(\mathrm{CH}), 108,7\left(\mathrm{C}_{\mathrm{q}}\right), 66.9(\mathrm{CH}), 59.5\left(\mathrm{CH}_{2}\right), 59.1(\mathrm{CH})$, $52.1\left(\mathrm{CH}_{2}\right), 47.8\left(\mathrm{CH}_{2}\right), 46.5\left(\mathrm{CH}_{2}\right), 42.6\left(\mathrm{CH}_{2}\right), 21.0\left(\mathrm{CH}_{3}\right), 20.5$ $\left(\mathrm{CH}_{3}\right)$. HRMS (ESI): $\mathrm{m} / \mathrm{z}$ : calc. for $\mathrm{C}_{46} \mathrm{H}_{43}{ }^{79} \mathrm{BrN}_{3}{ }^{+} 716.2640[\mathrm{M}+\mathrm{H}]^{+}$, found 716.2679, calc. for $\mathrm{C}_{39} \mathrm{H}_{39}{ }^{81} \mathrm{BrN}_{3}{ }^{+} 718.2620[\mathrm{M}+\mathrm{H}]^{+}$, found 718.2668 .

2-(naphthalen-2-ylmethyl)-11-phenyl-1-(3-(trifluoromethyl)phenyl)-10-(2,4,6-trimethylbenzyl)-2,3,8,9,10,11-hexahydro- $1 \mathrm{H}$-pyrazino[1',2':1,5]pyrrolo[4,3,2-de]isoquinoline $\mathbf{1 1 b}$

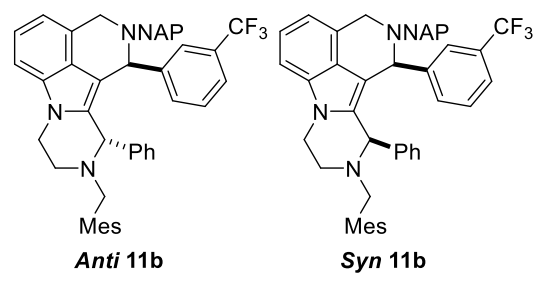

Compound 11b was synthesized following the General procedure 5 using tryptamine $\mathbf{5 d}(100 \mathrm{mg}$, $0.22 \mathrm{mmol}, \quad 1.0$ equiv.), 3-(trifluoromethyl)benzalde-

hyde ( $38 \mathrm{mg}, 0.22 \mathrm{mmol}, 1.0$ equiv.), Cat b $(16.0 \mathrm{mg}, 0.0217 \mathrm{mmol}$, $10 \mathrm{~mol} \%)$, M.S. $3 \AA ̊$ (104 mg), then benzaldehyde (46 mg, 0.43 mmol, 2.0 equiv.), in DCM $(3.0 \mathrm{~mL})$. The desired products $\mathbf{1 1} \mathbf{b}$ were obtained after column chromatography on silica gel (gradient from 0 to $10 \%$ Heptane:EtOAc) as an oil (98 mg, $0.12 \mathrm{mmol}$, $58 \%$, d.r 1:4 anti/syn). Anti 11b: IR (neat) $\mathbf{v}_{\max }=3057,2926,2852$, 1451, 1329, 1163, 1124, 747, $704 \mathrm{~cm}^{-1} .{ }^{1} \mathrm{H}$ NMR (CDCl $\left.3,500 \mathrm{MHz}\right)$ $\delta=7.88-7.84(\mathrm{~m}, 1 \mathrm{H}), 7.78(\mathrm{~d}, J=8.0 \mathrm{~Hz}, 2 \mathrm{H}), 7.56(\mathrm{~s}, 1 \mathrm{H}), 7.52-$ $7.45(\mathrm{~m}, 3 \mathrm{H}), 7.42(\mathrm{~d}, J=8.7 \mathrm{~Hz}, 1 \mathrm{H}), 7.33(\mathrm{~d}, J=7.8 \mathrm{~Hz}, 1 \mathrm{H}), 7.22$ $(\mathrm{s}, 1 \mathrm{H}), 7.24-7.08(\mathrm{~m}, 5 \mathrm{H}), 6.92(\mathrm{t}, J=7.4 \mathrm{~Hz}, 2 \mathrm{H}), 6.81-6.77(\mathrm{~m}, 3 \mathrm{H})$, $6.72(\mathrm{t}, J=7.3 \mathrm{~Hz}, 1 \mathrm{H}), 4.25(\mathrm{~s}, 1 \mathrm{H}), 4.19(\mathrm{td}, J=2.8,11.1 \mathrm{~Hz}, 1 \mathrm{H})$, $4.05(\mathrm{dt}, J=4.3,11.5 \mathrm{~Hz}, 1 \mathrm{H}), 3.82(\mathrm{~d}, J=16.1 \mathrm{~Hz}, 1 \mathrm{H}), 3.68-3.61$ $(\mathrm{m}, 2 \mathrm{H}), 3.56-3.46(\mathrm{~m}, 3 \mathrm{H}), 3.19(\mathrm{~d}, J=12.5 \mathrm{~Hz}, 1 \mathrm{H}), 3.15-3.10(\mathrm{~m}$, $1 \mathrm{H}), 2.73(\mathrm{dt}, J=3.5,11.5 \mathrm{~Hz}, 1 \mathrm{H}), 2.25(\mathrm{~s}, 3 \mathrm{H}), 2.19(\mathrm{~s}, 6 \mathrm{H}) .{ }^{13} \mathrm{C}\left\{{ }^{1} \mathrm{H}\right\}$ $\operatorname{NMR}\left(\mathrm{CDCl}_{3}, 125 \mathrm{MHz}\right) \delta=143.1\left(\mathrm{C}_{\mathrm{q}}\right), 139.5\left(\mathrm{C}_{\mathrm{q}}\right), 138.5\left(\mathrm{C}_{\mathrm{q}}\right), 137.3$ $\left(C_{q}\right), 136.7\left(C_{q}\right), 133.6\left(C_{q}\right), 133.1\left(C_{q}\right), 133.0\left(C_{q}\right), 133.0\left(C_{q}\right), 132.2$ $\left(\mathrm{C}_{\mathrm{q}}\right), 131.3\left(\mathrm{C}_{\mathrm{q}}\right), 130.2(\mathrm{CH}), 129.8(\mathrm{CH}), 129.2\left(\mathrm{q}, \mathrm{C}-\mathrm{F},{ }^{1} \mathrm{~J}_{\mathrm{C}-\mathrm{F}}=251.9\right.$ $\mathrm{Hz}, \mathrm{Cq}), 129.2(\mathrm{CH}), 128.3(\mathrm{CH}), 128.2\left(\mathrm{C}_{\mathrm{q}}\right), 128.1(\mathrm{CH}), 128.1(\mathrm{CH})$, $127.9(\mathrm{CH}), 127.9(\mathrm{CH}), 127.7(\mathrm{CH}), 127.2(\mathrm{CH}), 127.2(\mathrm{CH}), 126.1$ (q, C-F, $\left.{ }^{3} J_{\mathrm{C}-\mathrm{F}}=3.7 \mathrm{~Hz}, \mathrm{CH}\right), 125.9(\mathrm{CH}), 125.5(\mathrm{CH}), 125.5\left(\mathrm{C}_{\mathrm{q}}\right), 123.6$ $\left(q, C-F,{ }^{3} J_{C-F}=3.7 \mathrm{~Hz}, \mathrm{CH}\right), 122.0(\mathrm{CH}), 115.4(\mathrm{CH}), 106.8(\mathrm{CH}), 106.8$ $\left(\mathrm{C}_{\mathrm{q}}\right), 68.6(\mathrm{CH}), 59.4(\mathrm{CH}), 58.8\left(\mathrm{CH}_{2}\right), 52.3\left(\mathrm{CH}_{2}\right), 47.3\left(\mathrm{CH}_{2}\right), 47.3$ $\left(\mathrm{CH}_{2}\right), 43.1\left(\mathrm{CH}_{2}\right), 21.0\left(\mathrm{CH}_{3}\right), 20.6\left(\mathrm{CH}_{3}\right) .{ }^{19 F} \mathrm{NMR}\left(\mathrm{CDCl}_{3}, 282 \mathrm{MHz}\right)$ $\delta=-62.3$. HRMS (ESI): $\mathrm{m} / \mathrm{z}$ : calc for $\mathrm{C}_{47} \mathrm{H}_{43} \mathrm{~F}_{3} \mathrm{~N}_{3}{ }^{+} 706.3409[\mathrm{M}+\mathrm{H}]^{+}$, found 706.3380. Syn 11b: IR (neat) $\mathbf{v}_{\max }=3055,2924,2851,1451$, $1329,1162,1122,1073,748,699 \mathrm{~cm}^{-1} .{ }^{1} \mathrm{H} \mathrm{NMR}\left(\mathrm{CDCl}_{3}, 500 \mathrm{MHz}\right)$ $\delta=7.90-7.81(\mathrm{~m}, 3 \mathrm{H}), 7.66(\mathrm{~s}, 1 \mathrm{H}), 7.54-7.47(\mathrm{~m}, 3 \mathrm{H}), 7.25-7.14(\mathrm{~m}$, $3 \mathrm{H}), 7.00(\mathrm{t}, J=8.6 \mathrm{~Hz}, 1 \mathrm{H}), 6.85(\mathrm{~d}, J=7.9 \mathrm{~Hz}, 2 \mathrm{H}), 6.82-6.78(\mathrm{~m}$, $3 \mathrm{H}), 6.76(\mathrm{~s}, 1 \mathrm{H}), 6.74-6.69(\mathrm{~m}, 2 \mathrm{H}), 6.61(\mathrm{t}, J=7.6 \mathrm{~Hz}, 2 \mathrm{H}), 4.55(\mathrm{~s}$, $1 \mathrm{H}), 4.54(\mathrm{~s}, 1 \mathrm{H}), 4.23-4.16(\mathrm{~m}, 1 \mathrm{H}), 4.10(\mathrm{dt}, J=3.6,10.5 \mathrm{~Hz}, 1 \mathrm{H})$, $3.77(\mathrm{~d}, J=16.5 \mathrm{~Hz}, 1 \mathrm{H}), 3.72(\mathrm{~d}, J=12.4 \mathrm{~Hz}, 1 \mathrm{H}), 3.63(\mathrm{~d}, J=16.4$ $\mathrm{Hz}, 1 \mathrm{H}) ., 3.52-3.43(\mathrm{~m}, 2 \mathrm{H}), 3.24(\mathrm{~d}, J=12.5 \mathrm{~Hz}, 1 \mathrm{H}), 3.18-3.11(\mathrm{~m}$, $1 \mathrm{H}), 2.76$ (ddd, $J=3.9,10.1,11.9 \mathrm{~Hz}, 1 \mathrm{H}), 2.25(\mathrm{~s}, 3 \mathrm{H}), 2.18(\mathrm{~s}, 6 \mathrm{H})$. ${ }^{13} \mathrm{C}\left\{{ }^{1} \mathrm{H}\right\}$ NMR $\left(\mathrm{CDCl}_{3}, 125 \mathrm{MHz}\right) \delta=141.3\left(\mathrm{C}_{q}\right), 139.5\left(\mathrm{C}_{q}\right), 138.5$ $\left(C_{q}\right), 137.2\left(C_{q}\right), 136.7\left(C_{q}\right), 133.6\left(C_{q}\right), 133.2\left(C_{q}\right), 133.0\left(C_{q}\right), 132.0$ $\left(C_{q}\right), 131.8\left(C_{q}\right), 131.3\left(C_{q}\right), 129.5(C H), 129.2(C H), 128.3\left(C_{q}\right), 128.1$ (q, C-F, $\left.{ }^{1} J_{C-F}=275.8 \mathrm{~Hz}, C_{q}\right), 128.1(\mathrm{CH}), 127.8(\mathrm{CH}), 127.8(3 \mathrm{CH})$, $127.6(\mathrm{CH}), 127.6(\mathrm{CH}), 127.5(\mathrm{CH}), 126.1(\mathrm{CH}), 125.7(\mathrm{CH}), 125.7$ 
(CH), 125.5 (q, C-F, $\left.{ }^{3} \mathrm{~J}_{\mathrm{C}-\mathrm{F}}=3.5 \mathrm{~Hz}, \mathrm{CH}\right), 123.3\left(\mathrm{q}, \mathrm{C}-\mathrm{F},{ }^{3} \mathrm{~J}_{\mathrm{C}-\mathrm{F}}=3.8 \mathrm{~Hz}\right.$, CH), $122.0(\mathrm{CH}), 115.5(\mathrm{CH}), 106.9(\mathrm{CH}), 106.0\left(\mathrm{C}_{\mathrm{q}}\right), 67.5(\mathrm{CH}), 59.6$ $(\mathrm{CH}), 59.1\left(\mathrm{CH}_{2}\right), 52.2\left(\mathrm{CH}_{2}\right), 47.5\left(\mathrm{CH}_{2}\right), 46.8\left(\mathrm{CH}_{2}\right), 42.7\left(\mathrm{CH}_{2}\right), 21.0$ $\left(\mathrm{CH}_{3}\right), 20.6\left(\mathrm{CH}_{3}\right) .{ }^{19 F} \mathrm{NMR}\left(\mathrm{CDCl}_{3}, 282 \mathrm{MHz}\right) \delta=-62.3$. HRMS (ESI): $\mathrm{m} / \mathrm{z}$ : calc for $\mathrm{C}_{47} \mathrm{H}_{43} \mathrm{~F}_{3} \mathrm{~N}_{3}{ }^{+} 706.3409[\mathrm{M}+\mathrm{H}]^{+}$, found 706.3414 .

2-(naphthalen-2-ylmethyl)-11-phenyl-1-(quinolin-4-yl)-10-(2,4,6trimethylbenzyl)-2,3,8,9,10,11-hexahydro-1H-pyra-

zino[1',2':1,5]pyrrolo[4,3,2-de]isoquinoline $11 \mathrm{c}$
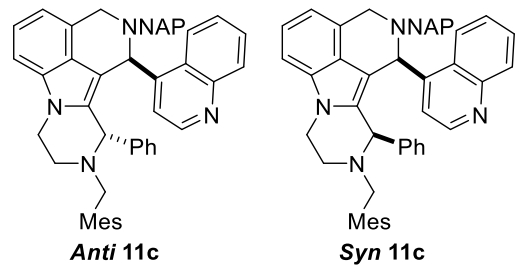

Compound 11c was synthesized following the General procedure 5 using tryptamine $5 \mathbf{d}$ (60 $\mathrm{mg}, 0.13$ mmol, 1.0 equiv.), quinoline-4-carbaldehyde $(20 \mathrm{mg}, 0.13$

mmol, 1.0 equiv.), Cat b (9.6 mg, $0.0130 \mathrm{mmol}, 10 \mathrm{~mol} \%)$, M.S. $3 \AA$ $(62 \mathrm{mg})$, at $40^{\circ} \mathrm{C}$ then benzaldehyde $(28 \mathrm{mg}, 0.26 \mathrm{mmol}, 2.0$ equiv.), in DCM (1.9 mL). The desired products $11 \mathrm{c}$ were obtained after column chromatography on silica gel (gradient from 0 to 35 $\%$ Heptane:EtOAc) as an oil (64 mg, $0.09 \mathrm{mmol}, 71 \%$, d.r 2:1 anti/syn). Anti 11c: IR (neat) $\mathbf{v}_{\max }=3056,2924,2850,1688,1591$, 1508, 1450, 1326, 1274, $758 \mathrm{~cm}^{-1} .{ }^{1} \mathrm{H}$ NMR $\left(\mathrm{CDCl}_{3}, 500 \mathrm{MHz}\right) \delta=$ $8.70(\mathrm{~d}, J=4.5 \mathrm{~Hz}, 1 \mathrm{H}), 8.17(\mathrm{~d}, J=8.6 \mathrm{~Hz}, 1 \mathrm{H}), 7.85-7.81(\mathrm{~m}, 1 \mathrm{H})$, 7.77-7.70 (m, 3H), $7.65(\mathrm{~d}, J=9.3 \mathrm{~Hz}, 1 \mathrm{H}), 7.55(\mathrm{~s}, 1 \mathrm{H}), 7.49-7.44$ $(\mathrm{m}, 2 \mathrm{H}), 7.44-7.37(\mathrm{~m}, 2 \mathrm{H}), 7.27(\mathrm{~d}, J=8.5 \mathrm{~Hz}, 1 \mathrm{H}), 7.22-7.13(\mathrm{~m}$, $4 \mathrm{H}), 7.04(\mathrm{t}, J=7.4 \mathrm{~Hz}, 2 \mathrm{H}), 6.82(\mathrm{~s}, 1 \mathrm{H}), 6.74(\mathrm{~d}, J=6.9 \mathrm{~Hz}, 1 \mathrm{H})$, $6.62(\mathrm{~d}, J=4.4 \mathrm{~Hz}, 1 \mathrm{H}), 4.33(\mathrm{~s}, 1 \mathrm{H}), 4.30-4.23(\mathrm{~m}, 2 \mathrm{H}), 4.15(\mathrm{dt}, J=$ $4.2,11.0 \mathrm{~Hz}, 1 \mathrm{H}), 3.62(\mathrm{~d}, J=18.3 \mathrm{~Hz}, 1 \mathrm{H}), 3.60(\mathrm{~s}, 2 \mathrm{H}), 3.56(\mathrm{~d}, J=$ $12.5 \mathrm{~Hz}, 1 \mathrm{H}), 3.47$ (d, J = 16.5 Hz, 1H), 3.23-3.16 (m, 2H), 2.81 (dt, $J=3.1,11.3 \mathrm{~Hz}, 1 \mathrm{H}), 2.27(\mathrm{~s}, 3 \mathrm{H}), 2.24(\mathrm{~s}, 6 \mathrm{H}) .{ }^{13} \mathrm{C}\left\{{ }^{1} \mathrm{H}\right\}$ NMR $\left(\mathrm{CDCl}_{3}\right.$, $125 \mathrm{MHz}) \delta=148.8(\mathrm{CH}), 147.7\left(\mathrm{C}_{\mathrm{q}}\right), 147.1\left(\mathrm{C}_{\mathrm{q}}\right), 138.9\left(\mathrm{C}_{\mathrm{q}}\right), 137.5$ $\left(\mathrm{C}_{\mathrm{q}}\right), 136.3\left(\mathrm{C}_{\mathrm{q}}\right), 135.7\left(\mathrm{C}_{\mathrm{q}}\right), 132.6\left(\mathrm{C}_{\mathrm{q}}\right), 132.4\left(\mathrm{C}_{\mathrm{q}}\right), 132.0\left(\mathrm{C}_{\mathrm{q}}\right), 131.9$ $\left(\mathrm{C}_{\mathrm{q}}\right), 130.1\left(\mathrm{C}_{\mathrm{q}}\right), 128.8(\mathrm{CH}), 128.6(\mathrm{CH}), 128.2(\mathrm{CH}), 128.1(\mathrm{CH})$, $127.5(\mathrm{CH}), 127.3(\mathrm{CH}), 126.9(\mathrm{CH}), 126.9(\mathrm{CH}), 126.7(\mathrm{CH}), 126.5$ $(\mathrm{CH}), 126.3\left(\mathrm{C}_{\mathrm{q}}\right), 125.0(\mathrm{CH}), 125.0(\mathrm{CH}), 124.7(\mathrm{CH}), 124.6(\mathrm{CH})$, 121.2 (CH), $120.3(\mathrm{CH}), 114.9(\mathrm{CH}), 106.0(\mathrm{CH}), 103.7\left(\mathrm{C}_{\mathrm{q}}\right), 81.7\left(\mathrm{C}_{\mathrm{q}}\right)$, $67.8(\mathrm{CH}), 58.4\left(\mathrm{CH}_{2}\right), 56.9(\mathrm{CH}), 51.4\left(\mathrm{CH}_{2}\right), 46.5\left(\mathrm{CH}_{2}\right), 44.6\left(\mathrm{CH}_{2}\right)$, $42.3\left(\mathrm{CH}_{2}\right), 20.0\left(\mathrm{CH}_{3}\right), 19.6\left(\mathrm{CH}_{3}\right)$. HRMS (ESI): $\mathrm{m} / \mathrm{z}$ : calc for $\mathrm{C}_{49} \mathrm{H}_{45} \mathrm{~N}_{4}{ }^{+} 689.3644[\mathrm{M}+\mathrm{H}]^{+}$, found 689.3622. Syn 11c: IR (neat) $\mathbf{v}_{\max }=3058,2924,2851,1694,1612,1508,1451,1345,1275,758$ $\mathrm{cm}^{-1} .{ }^{1} \mathrm{H}$ NMR $\left(\mathrm{CDCl}_{3}, 500 \mathrm{MHz}\right) \delta=8.38(\mathrm{~d}, J=4.2 \mathrm{~Hz}, 1 \mathrm{H}), 8.00-$ $7.86(\mathrm{~m}, 4 \mathrm{H}), 7.74(\mathrm{~s}, 1 \mathrm{H}), 7.57-7.50(\mathrm{~m}, 4 \mathrm{H}), 7.33-7.26(\mathrm{~m}, 2 \mathrm{H})$, $7.22(\mathrm{t}, J=7.5 \mathrm{~Hz}, 1 \mathrm{H}), 7.15(\mathrm{t}, J=7.6 \mathrm{~Hz}, 1 \mathrm{H}), 6.94-6.87(\mathrm{~m}, 2 \mathrm{H})$, $6.83(\mathrm{~d}, J=6.7 \mathrm{~Hz}, 1 \mathrm{H}), 6.81(\mathrm{~s}, 2 \mathrm{H}), 6.36(\mathrm{bs}, 1 \mathrm{H}), 6.22(\mathrm{t}, J=7.8$ $\mathrm{Hz}, 2 \mathrm{H}), 6.08(\mathrm{t}, J=7.3 \mathrm{~Hz}, 1 \mathrm{H}), 5.31(\mathrm{~s}, 1 \mathrm{H}), 4.65(\mathrm{~s}, 1 \mathrm{H}), 4.28-4.22$ $(\mathrm{m}, 1 \mathrm{H}), 4.15(\mathrm{dt}, J=4.0,10.9 \mathrm{~Hz}, 1 \mathrm{H}), 3.88(\mathrm{~d}, J=16.2 \mathrm{~Hz}, 1 \mathrm{H}), 3.78$ $(\mathrm{d}, J=12.7 \mathrm{~Hz}, 1 \mathrm{H}), 3.71(\mathrm{~d}, J=12.3 \mathrm{~Hz}, 1 \mathrm{H}), 3.63(\mathrm{~d}, J=16.5 \mathrm{~Hz}$, $1 \mathrm{H}), 3.45$ (d, $J=12.4 \mathrm{~Hz}, 1 \mathrm{H}), 3.25(\mathrm{~d}, J=12.1 \mathrm{~Hz}, 1 \mathrm{H}), 3.18(\mathrm{td}, J=$ $3.2,11.5 \mathrm{~Hz}, 1 \mathrm{H}), 2.83(\mathrm{dt}, J=3.8,11.3 \mathrm{~Hz}, 1 \mathrm{H}), 2.25(\mathrm{~s}, 3 \mathrm{H}), 2.20$ (6H). ${ }^{13} \mathrm{C}\left\{{ }^{1} \mathrm{H}\right\}$ NMR $\left(\mathrm{CDCl}_{3}, 125 \mathrm{MHz}\right) \delta=150.6\left(\mathrm{C}_{\mathrm{q}}\right), 149.1(\mathrm{CH})$, $148.4\left(C_{q}\right), 146.3\left(C_{q}\right), 139.3\left(C_{q}\right), 138.5\left(C_{q}\right), 136.9\left(C_{q}\right), 136.8\left(C_{q}\right)$, $133.5\left(C_{q}\right), 133.1\left(C_{q}\right), 133.0\left(C_{q}\right), 131.1\left(C_{q}\right), 130.4\left(C_{q}\right), 129.5(C H)$, $129.2(\mathrm{CH}), 129.2(\mathrm{CH}), 128.7(\mathrm{CH}), 128.4(\mathrm{CH}), 128.1(\mathrm{CH}), 127.9$ $(\mathrm{CH}), 127.9(\mathrm{CH}), 127.5(\mathrm{CH}), 127.0(\mathrm{CH}), 127.0\left(\mathrm{C}_{\mathrm{q}}\right), 126.3(\mathrm{CH})$, $126.0(\mathrm{CH}), 125.9\left(\mathrm{C}_{\mathrm{q}}\right), 125.7(\mathrm{CH}), 124.5(\mathrm{CH}), 122.3(\mathrm{CH}), 121.4$ $(\mathrm{CH}), 116.1(\mathrm{CH}), 107.0(\mathrm{CH}), 104.0\left(\mathrm{C}_{\mathrm{q}}\right), 68.9(\mathrm{CH}), 59.5\left(\mathrm{CH}_{2}\right), 54.7$ (CH), $52.6\left(\mathrm{CH}_{2}\right), 47.8\left(\mathrm{CH}_{2}\right), 47.4\left(\mathrm{CH}_{2}\right), 43.3\left(\mathrm{CH}_{2}\right), 21.0\left(\mathrm{CH}_{3}\right), 20.7$ $\left(\mathrm{CH}_{3}\right)$. HRMS (ESI): $\mathrm{m} / \mathrm{z}$ : calc for $\mathrm{C}_{49} \mathrm{H}_{45} \mathrm{~N}_{4}{ }^{+} 689.3644[\mathrm{M}+\mathrm{H}]^{+}$, found 689.3593.

\section{ASSOCIATED CONTENT}

\section{Supporting Information}

Details about optimization of the reactions, blank tests, NMR spectra, X-Ray Crystallography Data, Mechanistic Hypotheses. The Supporting Information is available free of charge on the ACS Publications website.

\section{AUTHOR INFORMATION}

\section{Corresponding Author}

* Xavier Guinchard: xavier.guinchard@cnrs.fr

\section{Author Contributions}

All authors have given approval to the final version of the manuscript.

\section{ACKNOWLEDGMENT}

Pierre Milcendeau and Nicolas Glinsky-Olivier thank MESRI (ParisSaclay University) for a PhD fellowship and the CHARMMMAT Laboratory of Excellence (ANR-11-LABX0039) for financial support. Zhenhao Zhang thanks the China Scholarship Council for PhD funding.

\section{REFERENCES}

1. See, for example: (a) Rahman, A.; Basha, A., Indole Alkaloids. Harwood accademic publishers: Reading, Berks, 1999. (b) Kawasaki, T.; Higuchi, K., Simple indole alkaloids and those with a nonrearranged monoterpenoid unit. Nat. Prod. Rep. 2005, 22, 761-793. (c) Gul, W.; Hamann, M. T., Indole alkaloid marine natural products: An established source of cancer drug leads with considerable promise for the control of parasitic, neurological and other diseases. Life Sciences 2005, 78, 442 453. (d) O'Connor, S. E.; Maresh, J. J., Chemistry and biology of monoterpene indole alkaloid biosynthesis. Nat. Prod. Rep. 2006, 23, 532-547.

2. For reviews, see: (a) Maity, P.; Adhikari, D.; Jana, A. K., An overview on synthetic entries to tetrahydro- $\beta$-carbolines. Tetrahedron 2019, 75, 965-1028. (b) Glinsky-Olivier, N.; Guinchard, X., Enantioselective Catalytic Methods for the Elaboration of Chiral Tetrahydro- $\beta$-carbolines and Related Scaffolds Synthesis 2017, 49, 2605-2620. (c) Cao, R.; Peng, W.; Wang, Z.; Xu, A., Beta-Carboline alkaloids: biochemical and pharmacological functions. Curr. Med. Chem. 2007, 14, 479-500. 3. (a) Pictet, A.; Spengler, T., On the formation of isochinolin-derivatives through the development of methylial on phenyl-aether, phenyl-alanin and tyrosin. Ber. Dtsch. Chem. Ges. 1911, 44, 2030-2036. (b) Tatsui, G., Über die Synthese von Carbolinderivaten. Yakugaku Zasshi 1928, 48, 453-459.

4. For reviews, see: (a) Calcaterra, A.; Mangiardi, L.; Delle Monache, G.; Quaglio, D.; Balducci, S.; Berardozzi, S.; lazzetti, A.; Franzini, R.; Botta, B.; Ghirga, F., The Pictet-Spengler Reaction Updates Its Habits. Molecules 2020, 25. (b) Gholamzadeh, P., Chapter Three - The Pictet-Spengler Reaction: A Powerful Strategy for the Synthesis of Heterocycles. In Advances in Heterocyclic Chemistry, Scriven, E. F. V.; Ramsden, C. A., Eds. Academic Press: 2019; Vol. 127, pp 153-226. (c) Rao, R. N.; Maiti, B.; Chanda, K., Application of Pictet-Spengler Reaction to Indole-Based Alkaloids Containing Tetrahydro- $\beta$-carboline Scaffold in Combinatorial Chemistry. ACS Comb. Sci. 2017, 19, 199-228. (d) Ingallina, C.; D'Acquarica, I.; Delle Monache, G.; Ghirga, F.; Quaglio, D.; Ghirga, P.; Berardozzi, S.; Markovic, V.; Botta, B., The PictetSpengler Reaction Still on Stage. Curr. Pharm. Des. 2016, 22, 1808-1850. (e) Dalpozzo, R., The Chiral Pool in the Pictet-Spengler Reaction for the Synthesis of $\beta$-Carbolines. Molecules 2016, 21, 699. (f) Bijoy, K.; Piyush, K. A.; Sudhir, K. S.; Devesh, S.; Anil, K. M.; Mohammad, S.; Sahaj, G., Pictet-Spengler Reaction Revisited: Engineering of Tetherd Biheterocycles into Annulated Polyheterocycles. Curr. Org. Synth. 2012, 9, 357-376. (g) Stockigt, J.; Antonchick, A. P.; Wu, F. R.; Waldmann, H., The PictetSpengler Reaction in Nature and in Organic Chemistry. Angew. Chem. Int. Ed. 2011, 50, 8538-8564. (h) Lorenz, M.; Van Linn, M. L.; Cook, J. M., The Asymmetric PictetSpengler Reaction. Curr. Org. Synth. 2010, 7, 189-223. (i) Cox, E. D.; Cook, J. M., The Pictet-Spengler condensation - a new direction for an old reaction. Chem. Rev. 1995, 95, 1797-1842.

5. (a) Zheng, C.; You, S.-L., Exploring the Chemistry of Spiroindolenines by Mechanistically-Driven Reaction Development: Asymmetric Pictet-Spengler-type Reactions and Beyond. Acc. Chem. Res. 2020, 53, 974-987. (b) Zheng, C.; Xia, Z.-L.; You, S.-L., Unified Mechanistic Understandings of Pictet-Spengler Reactions. Chem 2018, 4, 1952-1966. (c) Unsworth, W. P., Understanding the Role of Spiroindolenines in Pictet-Spengler Reactions. Chem 2018, 4, 1767-1770. (d) Kowalski, P.; Mokrosz, J. L., Structure and spectral properties of beta-carbolines .9. New arguments against direct rearrangement of the spiroindolenine intermediate into beta-carboline system in the Pictet-Spengler cyclization. An MNDO approach. Bull. Chem. Soc. Belg. 1997, 106, 147-149. (e) Kowalski, P.; Bojarski, A. J.; Mokrosz, J. L., Structure and spectral properties of $\beta$-carbolines. 8. Mechanism of the Pictet-Spengler cyclization: An MNDO approach. Tetrahedron 1995, 51, 2737-2742. (f) Bailey, P. D., Direct proof of the involvement of a spiro intermediate in the Pictet-Spengler reaction. J. Chem. Res. 
1987, 202-203. (g) Bailey, P. D., On the stereochemistry of the Pictet-Spengler reaction. Tetrahedron Lett. 1987, 28, 5181-5184. (h) Soerens, D.; Sandrin, J.; Ungemach, F.; Mokry, P.; Wu, G. S.; Yamanaka, E.; Hutchins, L.; Dipierro, M.; Cook, J. M., Study of the Pictet-Spengler reaction in aprotic media - Synthesis of the betagalactosidase inhibitor, Pyridinindolol. J. Org. Chem. 1979, 44, 535-545. (i) Gobé, V.; Gandon, V.; Guinchard, X., Reactions Involving Tryptamines and $\delta$-Allenyl Aldehydes: Competition between Pictet-Spengler Reaction and Cyclization to 1-Aminotetralins. Adv. Synth. Catal. 2018, 360, 1280-1288.

6. Lakhdar, S.; Westermaier, M.; Terrier, F.; Goumont, R.; Boubaker, T. Ofial, A. R.; Mayr, H., Nucleophilic Reactivities of Indoles. J. Org. Chem. 2006, 71 9088-9095.

$7 . \quad$ (a) Wu, F.; Zhu, H.; Sun, L.; Rajendran, C.; Wang, M.; Ren, X.; Panjikar, S.; Cherkasov, A.; Zou, H.; Stöckigt, J., Scaffold Tailoring by a Newly Detected PictetSpenglerase Activity of Strictosidine Synthase: From the Common Tryptoline Skeleton to the Rare Piperazino-indole Framework. J. Am. Chem. Soc. 2012, 134, 1498-1500. (b) Schönherr, H.; Leighton, J. L., Direct and Highly Enantioselective Iso-PictetSpengler Reactions with $\alpha$-Ketoamides: Access to Underexplored Indole Core Structures. Org. Lett. 2012, 14, 2610-2613. (c) Tiwari, R. K.; Verma, A. K.; Chhillar, A. K.; Singh, D.; Singh, J.; Kasi Sankar, V.; Yadav, V.; Sharma, G. L.; Chandra, R., Synthesis and antifungal activity of substituted-10-methyl-1,2,3,4-tetrahydropyrazino[1,2 a]indoles. Bioorg. Med. Chem. 2006, 14, 2747-2752. (d) Tiwari, R. K.; Singh, J.; Singh, D.; Verma, A. K.; Chandra, R., Highly efficient one-pot synthesis of 1-substituted1,2,3,4-tetrahydropyrazino[1,2-a]indoles. Tetrahedron 2005, 61, 9513-9518. (e) Katritzky, A. R.; Verma, A. K.; He, H.-Y.; Chandra, R., Novel Synthesis of 1,2,3,4 Tetrahydropyrazino[1,2-a]indoles. J. Org. Chem. 2003, 68, 4938-4940. (f) Huo, H.-r.; Tang, X.-Y.; Gong, Y.-f., Metal-Free Synthesis of Pyrrolo[1,2-a]quinoxalines Mediated by TEMPO Oxoammonium Salts. Synthesis 2018, 50, 2727-2740. (g) Xu, H.; Fan, L.-I., Synthesis and antifungal activities of novel 5,6-dihydro-indolo[1,2-a]quinoxaline derivatives. Eur. J. Med. Chem. 2011, 46, 1919-1925. (h) Preetam, A.; Nath, M., An eco-friendly Pictet-Spengler approach to pyrrolo- and indolo[1,2-a]quinoxalines using p-dodecylbenzenesulfonic acid as an efficient Brønsted acid catalyst. RCS Adv. 2015, 5, 21843-21853.

$8 . \quad$ (a) Voznesenskaia, N. G.; Shmatova, O. I.; Nenajdenko, V. G., Pictet-

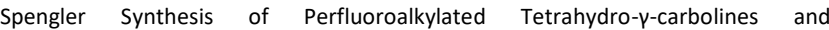
Tetrahydropyrrolopyrazines. Synthesis 2020, 52, 263-272. (b) Zhai, F.; Jordan, R. F., Complexation of an indole-based $\alpha$-aminoimine ligand to $\mathrm{Pd}(\mathrm{II})$. Inorg. Chim. Acto 2018, 482, 491-496. (c) Urmode, T. D.; Dawange, M. A.; Shinde, V. S.; Kusurkar, R. S. Synthesis of spiroindolone scaffolds by Pictet-Spengler spirocyclisation using $\beta$ cyclodextrin-SO3H as a recyclable catalyst. Tetrahedron 2017, 73, 4348-4354. (d) Riesco-Domínguez, A.; van der Zwaluw, N.; Blanco-Ania, D.; Rutjes, F. P. J. T., An Enantio- and Diastereoselective Mannich/Pictet-Spengler Sequence To Form Spiro[piperidine-pyridoindoles] and Application to Library Synthesis. Eur. J. Org Chem. 2017, 2017, 662-670. (e) Huang, L.; Dai, L.-X.; You, S.-L., Enantioselective Synthesis of Indole-Annulated Medium-Sized Rings. J. Am. Chem. Soc. 2016, 138 5793-5796. (f) Li, X.; Chen, D.; Gu, H.; Lin, X., Enantioselective synthesis of benzazepinoindoles bearing trifluoromethylated quaternary stereocenters catalyzed by chiral spirocyclic phosphoric acids. Chem. Commun. 2014, 50, 7538-7541. (g) Lee, Y.; Klausen, R. S.; Jacobsen, E. N., Thiourea-Catalyzed Enantioselective Iso-PictetSpengler Reactions. Org. Lett. 2011, 13, 5564-5567. (h) Grigg, R.; Sridharan, V.; Sykes, D. A., Sonogashira/ $\mathrm{N}$-acyliminium ion aromatic $\pi$-cyclisation processes: access to tetra- and pentacyclic lactams. Tetrahedron 2008, 64, 8952-8962.

9. Patil, N. T.; Shinde, V. S.; Sridhar, B., Relay Catalytic Branching Cascade: A Technique to Access Diverse Molecular Scaffolds. Angew. Chem. Int. Ed. 2013, 52, 2251-2255.

10. For other C4-iso Pictet-Spengler reactions, see: (a) Abe, T.; Yamada, K. Concise Syntheses of Hyrtioreticulins C and D via a C-4 Pictet-Spengler Reaction: Revised Signs of Specific Rotations. J. Nat. Prod. 2017, 80, 241-245. (b) Abe, T.; Haruyama, T.; Yamada, K., C4 Pictet-Spengler Reactions for the Synthesis of Core Structures in Hyrtiazepine Alkaloids. Synthesis 2017, 49, 4141-4150.

11. (a) Cera, G.; Bandini, M., Enantioselective Gold(I) Catalysis with Chiral Monodentate Ligands. Isr. J. Chem. 2013, 53, 848-855. (b) Obradors, C.; Echavarren A. M., Gold-Catalyzed Rearrangements and Beyond. Acc. Chem. Res. 2014, 47, 902912. (c) Zi, W.; Toste, D. F., Recent advances in enantioselective gold catalysis. Chem. Soc. Rev. 2016, 45, 4567-4589. (d) Ranieri, B.; Escofet, I.; Echavarren, A. M., Anatomy of gold catalysts: facts and myths. Org. Biomol. Chem. 2015, 13, 7103-7118. (e) Dorel, R.; Echavarren, A. M., Gold(I)-Catalyzed Activation of Alkynes for the Construction of Molecular Complexity. Chem. Rev. 2015, 115, 9028-9072. (f) Mascareñas, J. L.; Varela, I.; López, F., Allenes and Derivatives in Gold(I)- and Platinum(II)-Catalyzed Formal Cycloadditions. Acc. Chem. Res. 2019, 52, 465-479.

12. (a) Pirovano, V., Gold-Catalyzed Functionalization Reactions of Indole Eur. J. Org. Chem. 2018, 2018, 1925-1945. (b) Milcendeau, P.; Sabat, N.; Ferry, A Guinchard, X., Gold-catalyzed enantioselective functionalization of indoles. Org. Biomol. Chem. 2020, 18, 6006-6017.

13. (a) Zhang, Z.; Smal, V.; Retailleau, P.; Voituriez, A.; Frison, G.; Marinetti, A.; Guinchard, X., Tethered Counterion-Directed Catalysis: Merging the Chiral IonPairing and Bifunctional Ligand Strategies in Enantioselective Gold(I) Catalysis. J. Am. Chem. Soc. 2020, 142, 3797-3805. (b) Sabat, N.; Soualmia, F.; Retailleau, P.; Benjdia, A.; Berteau, O.; Guinchard, X., Gold-Catalyzed Spirocyclization Reactions of $\mathrm{N}$ Propargyl Tryptamines and Tryptophans in Aqueous Media. Org. Lett. 2020, 22, 4344 4349. (c) Magné, V.; Sanogo, Y.; Demmer, C. S.; Retailleau, P.; Marinetti, A.; Guinchard, X.; Voituriez, A., Chiral Phosphathiahelicenes: Improved Synthetic
Approach and Uses in Enantioselective Gold(I)-Catalyzed [2+2] Cycloadditions of $\mathrm{N}$ Homoallenyl Tryptamines. ACS Catal. 2020, 10, 8141-8148.

14. (a) Glinsky-Olivier, N.; Yang, S.; Retailleau, P.; Gandon, V.; Guinchard, X., Enantioselective Gold-Catalyzed Pictet-Spengler Reaction. Org. Lett. 2019, 21, 94469451. (b) Gobé, V.; Retailleau, P.; Guinchard, X., Self-Relay Gold(I)-Catalyzed PictetSpengler/Cyclization Cascade Reaction for the Rapid Elaboration of Pentacyclic Indole Derivatives. Chem. Eur. J. 2015, 21, 17587-17590.

15. For previous gold-catalyzed Picte-Spengler reactions, see: (a) Youn, S. W., Development of the Pictet-Spengler Reaction Catalyzed by $\mathrm{AuCl}_{3} / \mathrm{AgOTf}$. J. Org. Chem. 2006, 71, 2521-2523. (b) Subba Reddy, B. V.; Swain, M.; Reddy, S. M.; Yadav, J. S.; Sridhar, B., Gold-Catalyzed Domino Cycloisomerization/Pictet-Spengler Reaction of 2-(4-Aminobut-1-yn-1-yl)anilines with Aldehydes: Synthesis of Tetrahydropyrido[4,3-b]indole Scaffolds. J. Org. Chem. 2012, 77, 11355-11361.

16. Milcendeau, P.; Zhang, Z.; Glinsky-Olivier, N.; Van Elslande, E.; Guinchard, X., Au(I)-Catalyzed Pictet-Spengler Reactions: a Journey Around the Indole Ring. ChemRxiv 2020, doi 10.26434/chemrxiv.13296446.v1.

17. Mézailles, N.; Ricard, L.; Gagosz, F., Phosphine Gold(I) Bis(trifluoromethanesulfonyl)imidate Complexes as New Highly Efficient and Air-Stable Catalysts for the Cycloisomerization of Enynes. Org. Lett. 2005, 7, 4133-4136.

18. (a) Jónsson, H. F.; Fiksdahl, A., Studies on gold-nitrone systems. Dalton Trans. 2019, 48, 142-149. (b) Jónsson, H. F.; Evjen, S.; Fiksdahl, A., Gold(I)-Catalyzed [2+2 + 2] Cyclotrimerization of 1,3-Diarylpropargyl Acetals. Org. Lett. 2017, 19, 2202 (c) Evjen, S.; Fiksdahl, A., Gold(I)-catalysed [3+3] cycloaddition of propargyl acetals and nitrones. Tetrahedron 2016, 72, 3270-3276.

19. The conversion rate was $65 \%$. The same reaction without catalyst led to a lower $25 \%$ conversion over $60 \mathrm{~h}$.

20. Both syn and anti diastereomers of the series have a typical footprint in ${ }^{1} \mathrm{H}$ NMR spectra. NOESY experiments and Xray obtained in other compounds of the series allowed the determination of the relative configurations of all the products by analogy.

21. (a) Martinez, C. R.; Iverson, B. L., Rethinking the term "pi-stacking". Chem. Sci. 2012, 3, 2191-2201. (b) Cozzi, F.; Ponzini, F.; Annunziata, R.; Cinquini, M.; Siegel, J. S., Polar Interactions between Stacked $\pi$ Systems in Fluorinated 1,8 Diarylnaphthalenes: Importance of Quadrupole Moments in Molecular Recognition. Angew. Chem. Int. Ed. 1995, 34, 1019-1020. (c) Hunter, C. A.; Sanders, J. K. M., The nature of .pi.-.pi. interactions. J. Am. Chem. Soc. 1990, 112, 5525-5534. (d) Wheeler S. E.; Houk, K. N., Substituent Effects in the Benzene Dimer are Due to Direct Interactions of the Substituents with the Unsubstituted Benzene. J. Am. Chem. Soc. 2008, 130, 10854-10855.

22. Previous papers have hypothesized the potential involvement of $A u(I)$ complexes and/or acidic protons in the formation of iminium from hemiaminal in related $\mathrm{Au}(\mathrm{I})$-catalyzed reactions. However, our own results (see ref $14 \mathrm{a}$ ) ruled out the possibility that protons were involved in that type of elementary step because reactions were enantioselective. For these reports, see ref 9 and: a) J. Qiao, X. Jia, P. Li, X. Liu, J. Zhao, Y. Zhou, J. Wang, H. Liu, F. Zhao, Adv. Synth. Catal. 2019, 361, 1419; b) N. T. Patil, A. K. Mutyala, P. G. Lakshmi, B. Gajula, B. Sridhar, G. R. Pottireddygari, T. P. Rao, J. Org. Chem. 2010, 75, 5963; c) T. Yang, L. Campbell, D. J. Dixon, J. Am Chem. Soc. 2007, 129, 12070.

23. Schlegel, M.; Coburger, P.; Schneider, C., A Novel Sc(OTf) ${ }_{3}$-Catalyzed $(2+2+1)$-Cycloannulation/Aza-Friedel-Crafts Alkylation Sequence toward Multicyclic 2-Pyrrolines. Chem. Eur. J. 2018, 24, 14207-14212.

24. Enders, D.; Wang, C.; Yang, X.; Raabe, G., One-Pot Organocatalytic Asymmetric Synthesis of $1 \mathrm{H}$-Pyrrolo[1,2a]indol-3(2H)-ones via a MichaelHemiaminalization-Oxidation Sequence. Synlett 2011, 2011, 469-472.

25. Robaa, D.; Enzensperger, C.; Abul Azm, S. E. D.; El Khawass, E. S.; E Sayed, O.; Lehmann, J., Dopamine Receptor Ligands. Part 18: Modification of the Structural Skeleton of Indolobenzazecine-Type Dopamine Receptor Antagonists. J. Med. Chem. 2010, 53, 2646-2650. 